\title{
Republic of Mozambique: Fourth Review Under the Policy Support Instrument and Request for Modification of Assessment Criteria—Staff Report; Debt Sustainability Analysis; Press Release on the Executive Board Discussion; and Statement by the Executive Director for Mozambique.
}

In the context of the fourth review under the policy support instrument and request for modification of assessment criteria, the following documents have been released and are included in this package:

- $\quad$ The staff report for the fourth review under the Policy Support Instrument and request for modification of assessment criteria, prepared by a staff team of the IMF, following discussions that ended on March 16, 2012, with the officials of Mozambique on economic developments and policies. Based on information available at the time of these discussions, the staff report was completed on May 11, 2012. The views expressed in the staff report are those of the staff team and do not necessarily reflect the views of the Executive Board of the IMF.

- $\quad$ A staff supplement on the Debt Sustainability Analysis dated May 11, 2012.

- $\quad$ A Press Release summarizing the views of the Executive Board as expressed during its June 6, 2012 discussion of the staff report that completed the request and/or review.

- $\quad$ A statement by the Executive Director for the Republic of Mozambique.

The documents listed below have been or will be separately released.

Letter of Intent sent to the IMF by the authorities of Mozambique* Memorandum of Economic and Financial Policies by the authorities of Mozambique*

*Also included in Staff Report

The policy of publication of staff reports and other documents allows for the deletion of market-sensitive information.

Copies of this report are available to the public from

International Monetary Fund • Publication Services

$70019^{\text {th }}$ Street, N.W. • Washington, D.C. 20431

Telephone: (202) 623-7430 • Telefax: (202) 623-7201

E-mail: publications@imf.org Internet: http://www.imf.org

\section{International Monetary Fund Washington, D.C.}




\section{INTERNATIONAL MONETARY FUND}

\section{REPUBLIC OF MOZAMBIQUE}

\section{FOURTH REVIEW UNDER THE POLICY SUPPORT}

May 11, 2012

\section{INSTRUMENT AND REQUEST FOR MODIFICATION OF ASSESSMENT CRITERIA}

\section{KEY ISSUES}

Context and policy challenges. Mozambique's macroeconomic performance remains strong and program implementation satisfactory. Economic growth in 2011 was buoyant and inflation fell sharply, but the short-term outlook entails elevated risks from the weakening global economy. Stagnant poverty reduction calls for a swift implementation of the recently adopted Poverty Reduction Strategy (PARP) to generate more inclusive growth.

Short-term policy framework. The main short-term challenge is to mitigate the impact of the global crisis while safeguarding the gains from the authorities' recent disinflation effort. A countercyclical macroeconomic policy mix is warranted in 2012: On the fiscal policy side, automatic stabilizers will be allowed to work to maintain spending at budgeted levels, which will entail a slight increase in the fiscal deficit. The Bank of Mozambique (BM) will maintain an accommodating monetary policy to support higher credit growth to the private sector, while monitoring inflation developments closely. An updated assessment of financial sector vulnerabilities suggests that banks have some buffers to withstand contagion from the euro area crisis, but the BM should step up its supervision and ensure the completion of the financial sector contingency plan.

Medium-term challenges. Given the declining trend in foreign aid in the medium term, the government is tapping more into nonconcessional borrowing to close the infrastructure gap. Further strengthening debt management and investment planning is crucial for informed decision-making, while tapping the abundant natural resource wealth will help create the necessary fiscal space to support the structural transformation of the economy in the long run. To promote inclusive growth, the government has launched a series of initiatives to implement the PARP, including an overhaul of social protection programs. Reform momentum needs to be maintained, as implementation challenges are enormous and administrative capacity and coordination need to be upgraded. 


\section{Approved By}

Saul Lizondo and

Vivek Arora
A staff team comprising Messrs. Mueller (head), Gitton, Xiao (all AFR), Ms. Garcia (SPR), and Messrs. Hesse (MCM) and Hoffmann (MCM expert) visited Maputo during March 5-16, 2012. The mission met with Minister Chang (Finance), Cuereneia (Planning and Development), and other line ministers (Energy, Agriculture, Natural Resources, Industry and Trade, Women and Social Action), BM Governor Gove, and other senior government officials. The mission also met with development partners, civil society, academia, and the private sector. It was assisted by Mr. Lledo (resident representative), Mr. Wane, and Ms. Palacio (resident representative office). Messrs. Conceição (OED) and Clarke and Revilla (World Bank) participated in the policy discussions.

\section{CONTENTS}

I. BACKGROUND AND RECENT DEVELOPMENTS

II. ECONOMIC OUTLOOK AND POLICY DISCUSSIONS..........................................................9

A. Short-Term Challenge: Mitigating the Impact of the Global Slowdown …………….............9

B. Medium-Term Policies: Promoting InCLUSiVE ANd SuStainABLE GROWTH .................................... 16

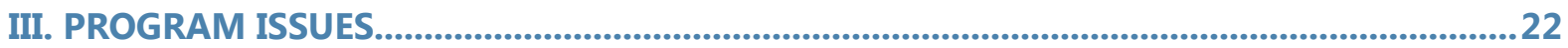

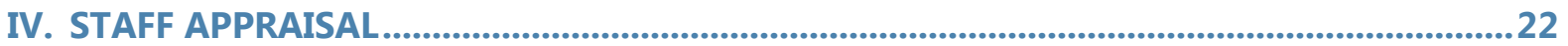

\section{FIGURES}

1. Economic Developments in the Global context.....................................................................................

2. Inflation and Monetary Developments.................................................................................................

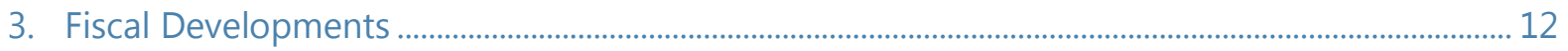

\section{BOXES}

1. Banking Vulnerabilities and Financial Sector Regulation...................................................................... 15

2. Identifying Fiscal Space for a Social Protection Floor in Mozambique.............................................. 20

\section{TABLES}

1. Selected Economic and Financial Indicators, 2009-17......................................................................... 24

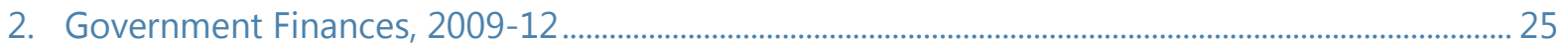

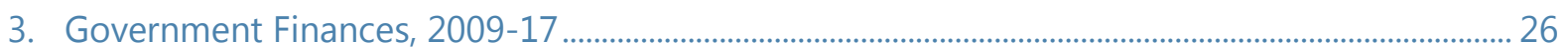

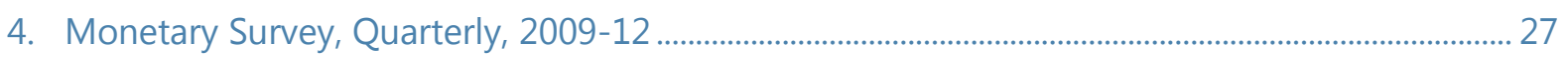

6. Financial Soundness Indicators for Banking Sector, 2001-11 .............................................................. 29

7. Quantitative Assessment Criteria and Indicative Targets ...................................................................... 30

8. Structural Conditionality Under the Current PSI, 2011-12 ………………………………................... 33 


\section{APPENDICES}

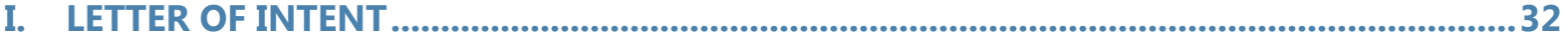

ATTACHMENT 1: Memorandum of Economic and Financial Policies ............................................ 34

ATTACHMENT 2: Technical Memorandum of Understanding .......................................................... 50

II. RESERVE ADEQUACY ASSESSMENT .................................................................................5 57 


\section{BACKGROUND AND RECENT DEVELOPMENTS}

1. The Mozambican economy recorded a strong performance in 2011, showing little sign of being affected by the global turmoil. Preliminary data indicate that GDP rose by about 7 percent, comparing favorably with the growth rates of Mozambique's peers. Growth was broad-based, with agriculture, mining, transportation and communication, and financial services registering the fastest expansion. Activity was also supported by strong exports and robust investment demand from megaprojects in the natural resource sector and the public sector.

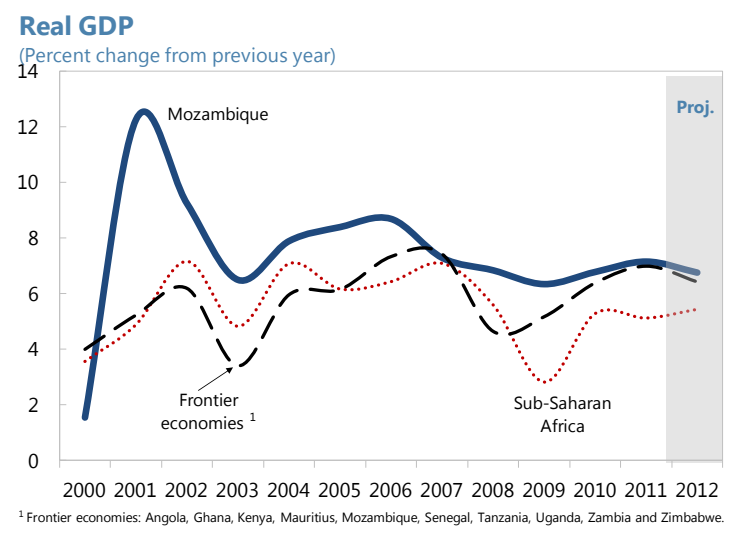

\section{The authorities' disinflation policies}

yielded commendable results. Twelve-month inflation decelerated sharply to $23 / 4$ percent in March 2012, compared to $16 \frac{1}{2} 2$ percent at end2010. Core inflation, once hovering in the double-digit range, fell to $2 \frac{1}{3}$ percent in March. This reflects a determined monetary tightening during 2010-11, which decelerated broad money growth by two-thirds in 2011 from a year earlier and contributed to the appreciation of the metical relative to main partners' currencies. An appropriately restrictive fiscal policy stance, especially in the first half of 2011, was also conducive to disinflation, as were favorable trends in international food and energy prices.

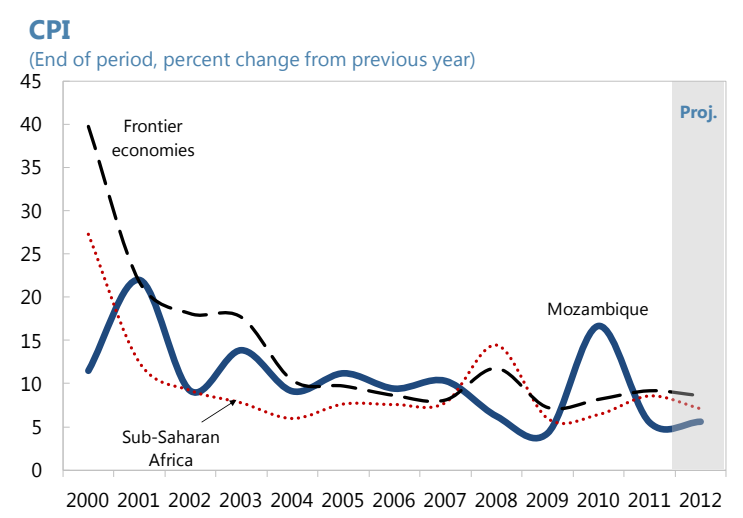

3. The external sector performed well, and Mozambique's net international reserves (NIR) were further buttressed. Preliminary data for 2011 suggest that both megaprojects' and traditional exports were buoyant, although strong import demand for investment goods resulted in a slight worsening of the current account deficit, to 13 percent of GDP. Led by megaprojects, FDI more than doubled relative to 2010 , which supported an NIR accumulation of US\$331 million and a comfortable reserve coverage (5.2 months of current-year imports). 
FDI by Sector, 2011

(Share of total, percent)

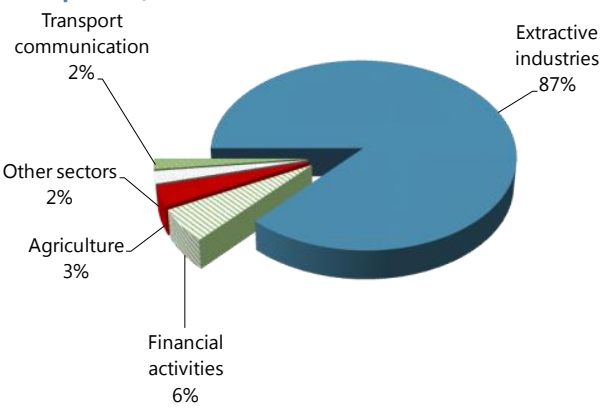

Source: Mozambican authorities.

Strong capital inflows and the tight monetary policy stance contributed to a 20 percent real appreciation of the metical in 2011, after a real depreciation of 15 percent a year earlier.

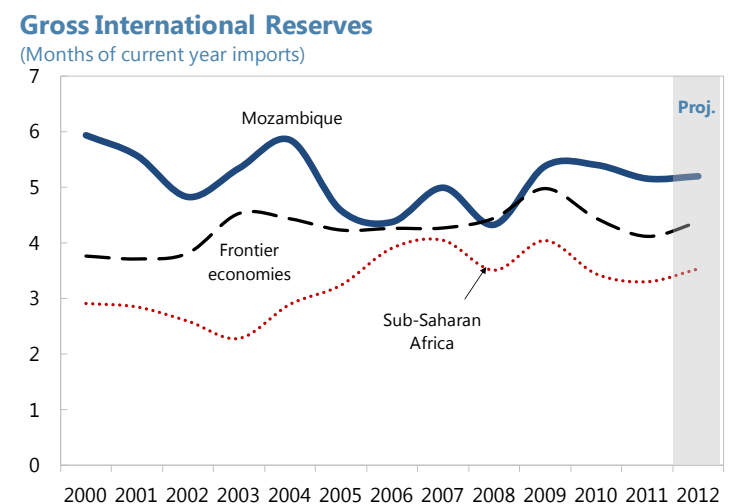

\section{Program performance remains} satisfactory, and all but one of the end2011 assessment criteria (ACs) were met. In particular:

- In line with the disinflation policy stance, the quantitative targets for reserve money (RM) and NIR were met comfortably.

- Net credit to the government (NCG) exceeded the end-2011 AC marginally (0.1 percent of GDP) due to an unexpected calling of a government guarantee for a public enterprise (0.2 percent of GDP). While the domestic primary deficit was substantially lower than expected on account of a strong revenue performance and domestic spending below budgeted levels, the overall fiscal deficit was $1 / 2$ percent of GDP higher than envisaged due to a rapid pick-up of donor-financed capital spending in the second half of the year. Encouragingly, priority spending exceeded its end-year indicative floor, after missing the targets repeatedly in the past.

- Since the inception of the PSI-supported program in June 2010, the government has used US $\$ 446$ million of its US $\$ 900$ million limit on nonconcessional borrowing (NCB). Since the last review, a US\$300million loan was signed with China to build a ring road around Maputo. The loan was expected by both parties to be concessional, but it became nonconcessional by a small margin as the discount rate applied in the concessionality calculations changed during the loans' final negotiation phase.

\section{Program Performance}

\begin{tabular}{l}
\hline \\
Assessment Criteria \\
Net credit to the government \\
Indicative
\end{tabular}

met $\triangle$ not met 


\section{The authorities continue to make} progress in structural reforms. The structural benchmark on the approval of the Integrated Investment Program for March 2012 was observed. The plan sets up a strategic vision across sectors and identifies some of the criteria to be used for project selection, although more specificity on the technical criteria that will be used for identifying, evaluating, and ranking projects would have been desirable. The plan lists about two dozen projects which the authorities currently consider as infrastructure investment priorities while emphasizing that the document is a rolling document that would be updated in light of further technical work. The authorities also adopted five of the six modules under the financial sector contingency plan, an endNovember 2011 benchmark. However, the mission, based on the assessment by participating MCM staff, recommended enhancements to three modules, which the authorities agreed to incorporate over the next few months. The authorities appear on course to meet the program's forthcoming structural benchmarks. 


\section{Figure 1. Mozambique: Economic Developments in the Global Context}

The global weakness has yet to have a significant impact on Mozambique's economic growth.

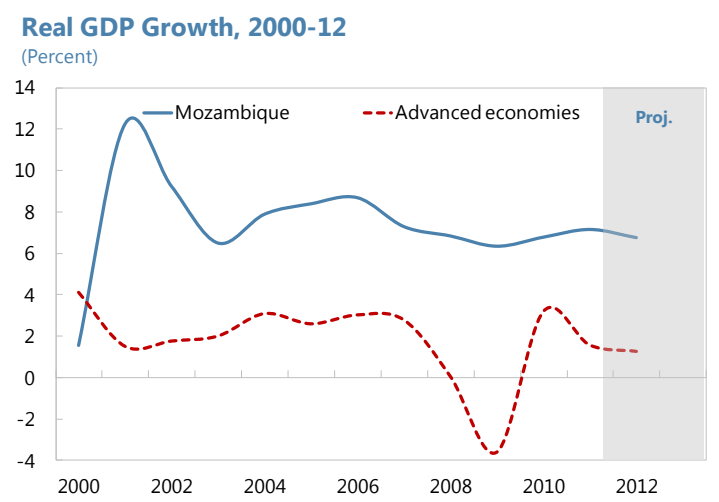

...while strong capital inflows and the monetary tightening have contributed to the appreciation of the metical.

Nominal and Real Effective Exchange Rates (Index, Average 2005=100)

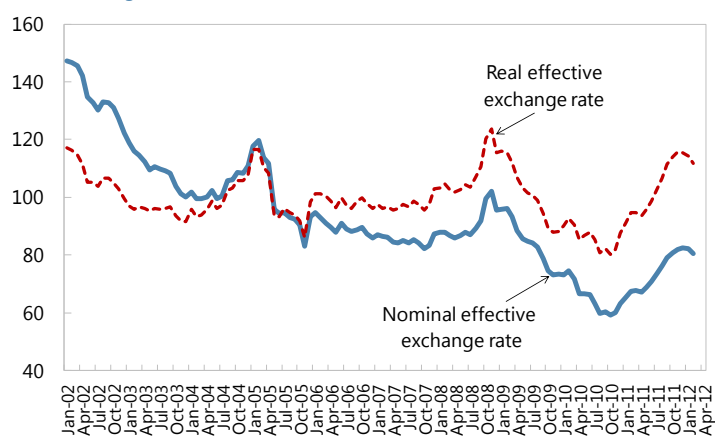

The current account deficit has widened somewhat, as megaprojects have resumed dividend payments and remittances outflows have risen.

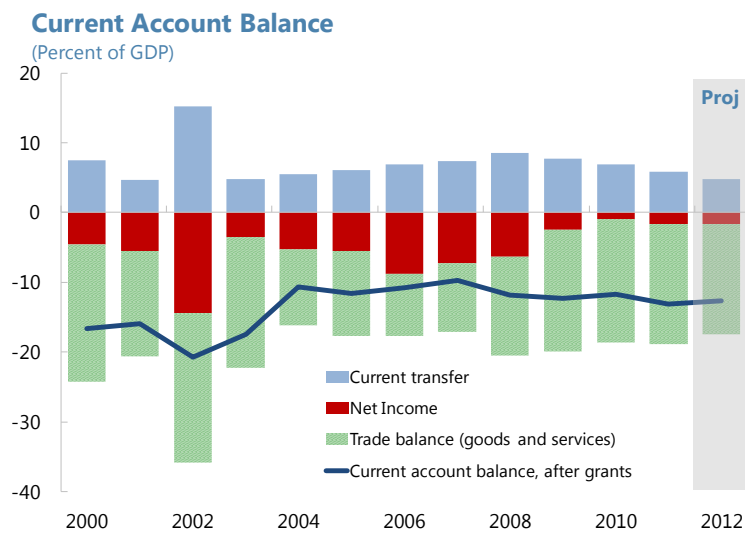

Over the last two years, the terms of trade have improved,...

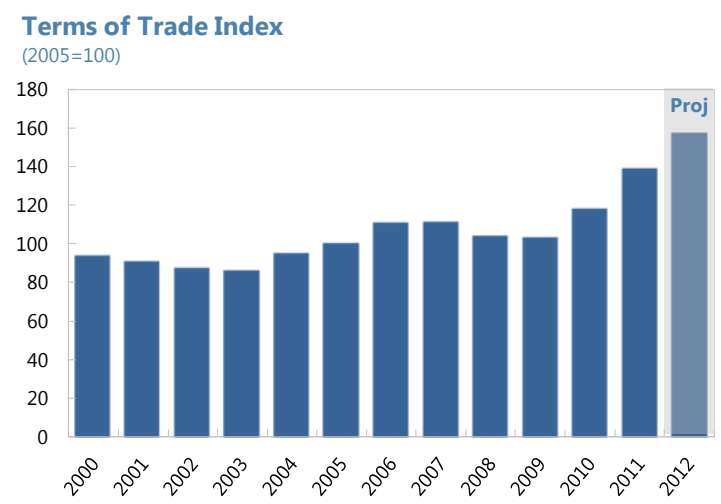

Megaprojects' strong export growth was counterbalanced by investment-related imports and fuel imports.

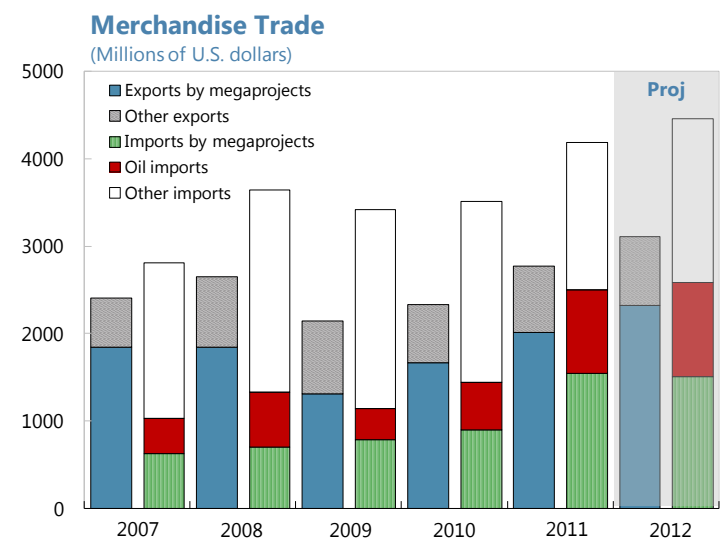

Strong private capital inflows especially by megaprojects have helped to maintain the reserve cover.

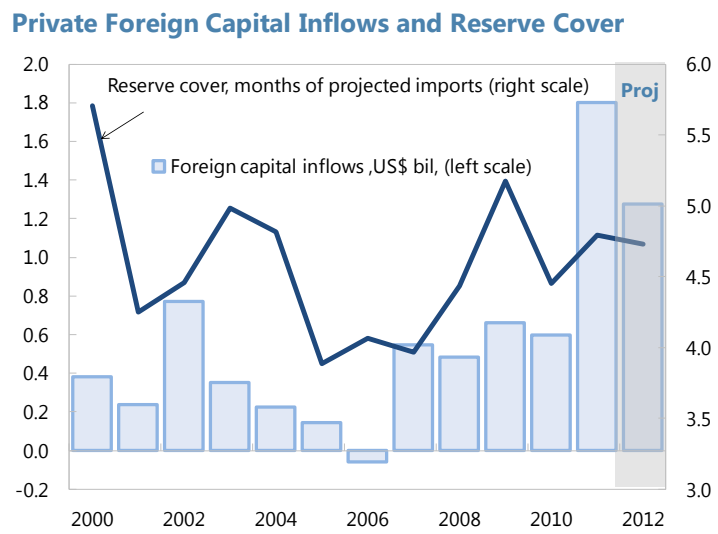

Sources: Mozambican authorities and IMF staff estimates and projections. 


\section{Figure 2. Mozambique: Inflation and Monetary Developments}

Both headline and core inflation fell sharply since early $2011, \ldots$

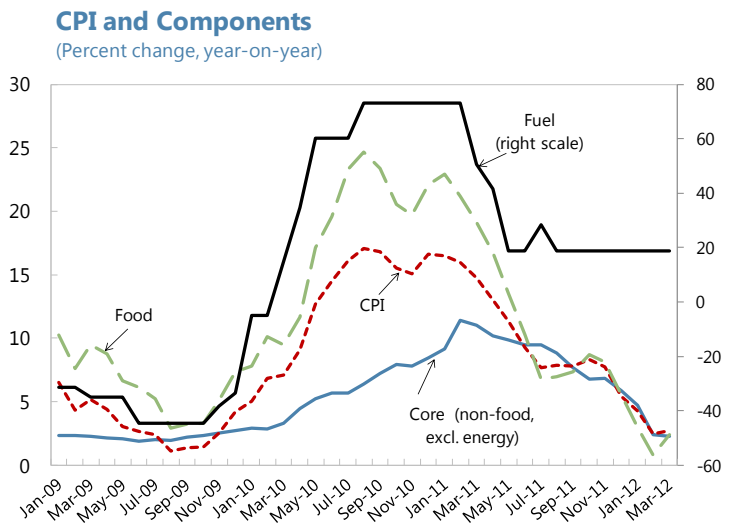

...determined monetary tightening which reined in broad money and credit growth, ...

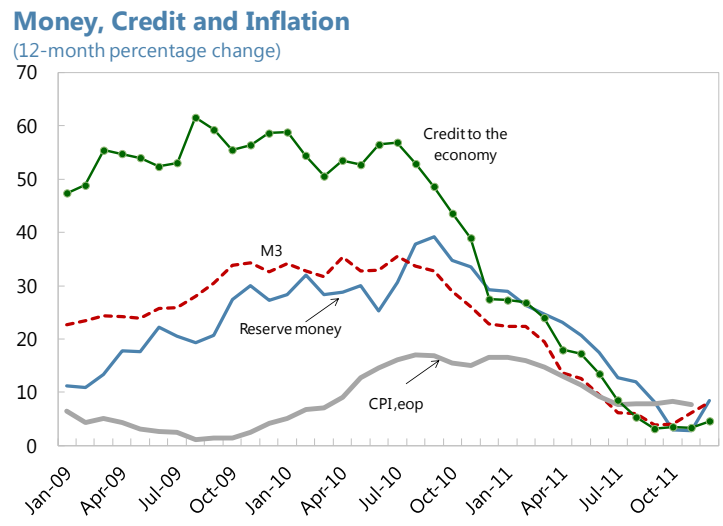

Following the monetary tightening in 2010-11, the BM began to lower rates in late 2011.

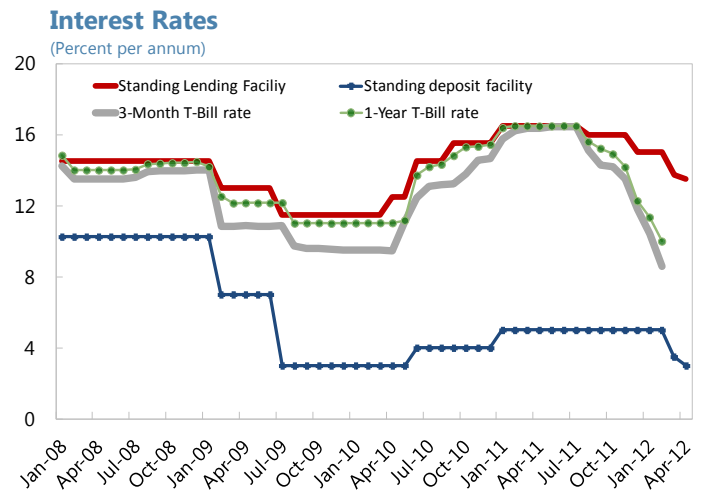

...helped by favorable developments in international prices and...

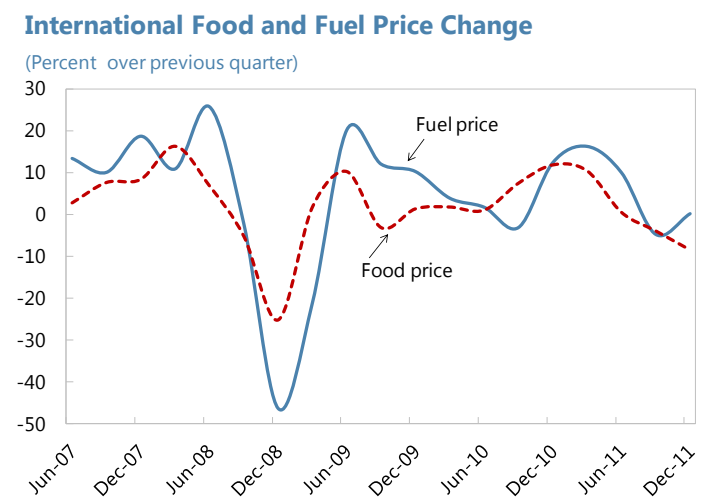

...which contributed to the metical's appreciation.

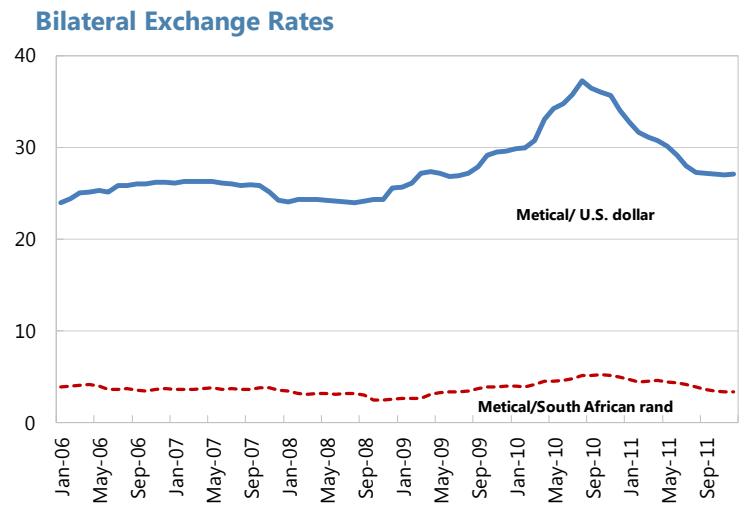

Reserve money was kept in line with program targets, helping to achieve the disinflation objective.

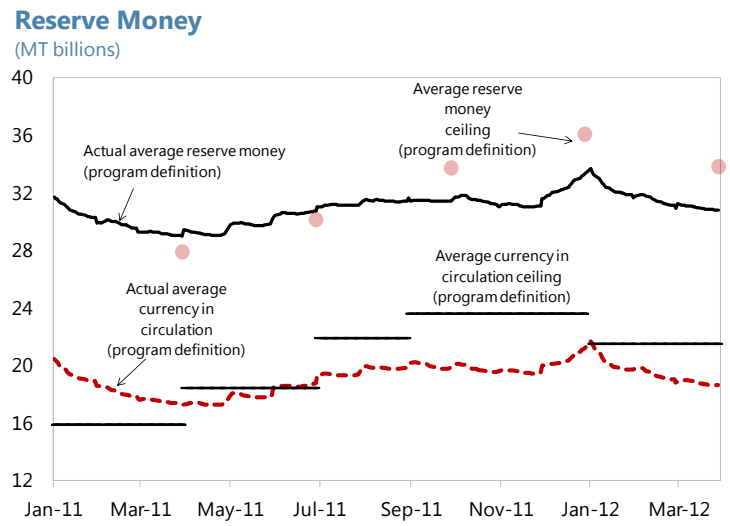

Sources: Mozambican authorities and IMF staff estimates and projections. 


\section{ECONOMIC OUTLOOK AND POLICY DISCUSSIONS}

6. Discussions focused on the impact of the global downturn on Mozambique, the appropriate policy response, and the medium- term reform agenda to achieve inclusive and sustainable growth and accelerate poverty reduction.

\section{A. Short-Term Challenge: Mitigating the Impact of the Global Slowdown}

\section{The worsened global economic}

\section{outlook for 2012-13 poses a risk to}

Mozambique's economic performance.

During the 2008-09 global crisis,

Mozambique's economic growth fell below trend, largely due to a decline in FDI and the collapse in world commodity markets.

However, a countercyclical policy stance, stepped-up donor support, and a domestic banking system that compensated for a shortfall in external financing helped support economic activity at the crucial juncture.

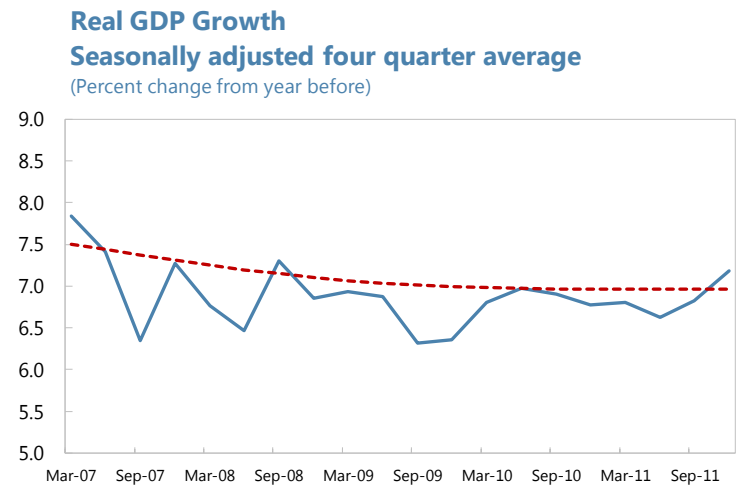

The current global turmoil could affect

Mozambique through somewhat different channels. While a weakening export demand and a capital flow reversal could possibly recur, the difficulties in the euro area raise the specter of lower aid disbursements and more limited credit availability, the latter due to deleveraging of banks and potential spillovers from the European financial sector. Although Mozambique has not felt the brunt of these factors to date, they are likely to come to the fore in 2012. On the other hand, the partial removal of transportation bottlenecks in the booming coal-exporting industry should enable an improved performance of this sector this year, thereby mitigating the impact of the global shock.

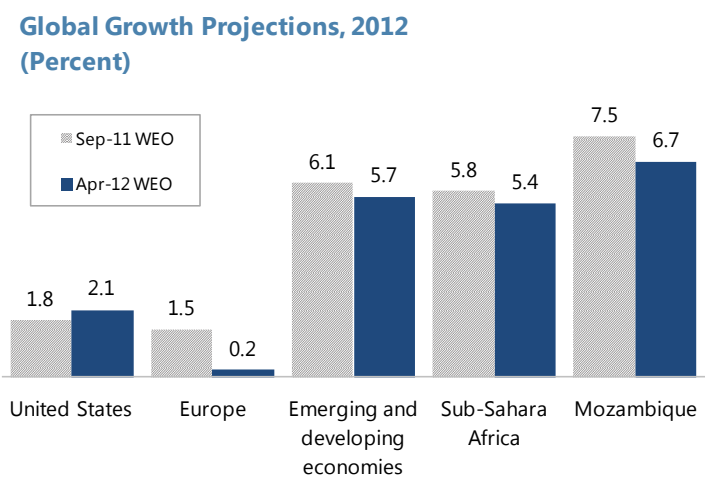

\section{To help cushion the impact of a temporary global slowdown in 2012-13,}




\section{staff and the authorities reached}

\section{understandings on a countercyclical policy}

mix for the short term. On the fiscal side, the government will allow automatic stabilizers to function to support domestic demand. The BM will maintain the accommodative monetary policy stance it started in late 2011 seeking to balance the need to provide additional credit to the private sector with safeguarding the recent disinflation gains. This macroeconomic policy mix will be complemented by intensified

\section{Fiscal Policy}

\section{The operation of automatic} stabilizers will help implement a countercyclical fiscal policy stance in 2012.

In light of the possibly less favorable economic environment, the baseline scenario MEFP ๆ14-15 reflects lower revenue collections than in past projections that had been based on the 2012 budget law. However, the government intends to maintain its spending program to avoid aggravating the economic and social effects of the global downturn and to pursue the country's transformation agenda under the PARP. As a result, the domestic primary deficit could rise by 0.2 percentage points relative to earlier projections, to 2.3 percent of GDP. This higher deficit would be fully financed by a topping-up of World Bank assistance, thus avoiding financial sector supervision, aiming at guarding against potential cross-border contagion. As a result, economic growth is expected to slow to a still robust $63 / 4$ percent in 2012 , while inflation should remain well in single digits. Both the external current account deficit and international reserve cover are expected to remain similar to 2011, as somewhat weaker export growth is expected to be offset by slowing FDI-related imports.

crowding out the private sector from domestic bank financing. A larger-than-projected drop in revenues would, however, trigger application of the provisions which limit budget execution to cautionary ceilings consistent with the Organic Budget Law (SISTAFE 2002).

Mozambique: Key Fiscal Indicators

\begin{tabular}{lrrrr}
\hline & 2011 & \multicolumn{2}{c}{2012} & 2013 \\
\cline { 2 - 5 } & \multicolumn{2}{c}{ Est. CR 11/350 } & Proj. & Proj. \\
\hline & (Percent of GDP, & unless otherwise & stated) \\
Overall balance after grants & -5.2 & -5.9 & -6.4 & -6.5 \\
Domestic primary balance & -2.2 & -2.1 & -2.3 & -2.1 \\
Net domestic financing & 0.9 & 0.5 & 0.6 & 0.7 \\
Memorandum items: & & & & \\
Domestic demand impact (change & & & & \\
from the previous year) 1/ & -2.0 & -0.2 & 0.6 & 0.2 \\
Real GDP growth (percent) & 7.1 & 7.5 & 6.7 & 7.2 \\
Average inflation (percent) & 10.4 & 7.2 & 7.2 & 5.6 \\
\hline
\end{tabular}

Sources: Mozambican authorities and IMF staff estimates and projections. $1 /$ The overall balance overstates the domestic impact of capital spending as the latter has a significant import content. This calculation attempts to adjust the overall balance for this bias. 


\section{The government reiterated its} commitment to preserve the quality of spending.

- Safeguarding priority spending. The government will channel at least half of all MEFP \17 spending (excluding net lending) toward the priority sectors under the PARP.

- Containing growth of the wage bill. The government will decelerate the recruitment in non-priority areas and the

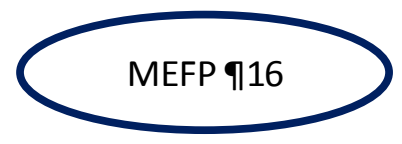
pace of decompression of the pay scale that was behind the rise of the wage bill over the last five years. It will also strengthen its monitoring of the wage bill through a full roll-out of the electronic salary payment system (e-FOLHA) to all provincial directorates by end-2012 (structural benchmark).

- Phasing out the fuel subsidy. While cost recovery was effectively achieved early in 2012, the subsequent global oil price increases have again caused a need for compensation for fuel importers.

The mission

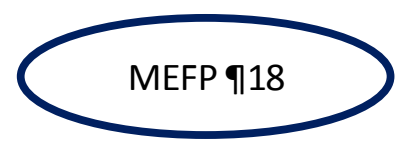
welcomed the achievements so far, but urged the authorities to show more resolve going forward in avoiding a renewed build-up of large fuel subsidies. It agreed that further exploring options for cross- subsidization of various petroleum products through greater segmentation of the market and making relatively more frequent and smaller price adjustments than in the past could effectively contain the fiscal risks. With a more medium-term perspective, the government should review avenues to improve the price-setting formula that has temporarily been suspended. As agreed with fuel importers, the government will complete the payment of compensation for their losses incurred in 2011 by mid-2012. 
Figure 3. Mozambique: Fiscal Developments

As a result of recent reforms in tax administration, revenue collections continue to be buoyant,...

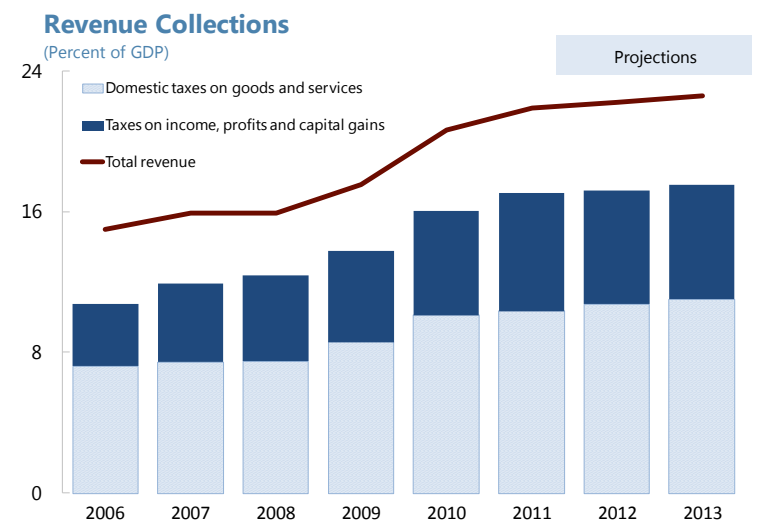

Capital spending relies heavily on external project support, which has been on a declining trend,...

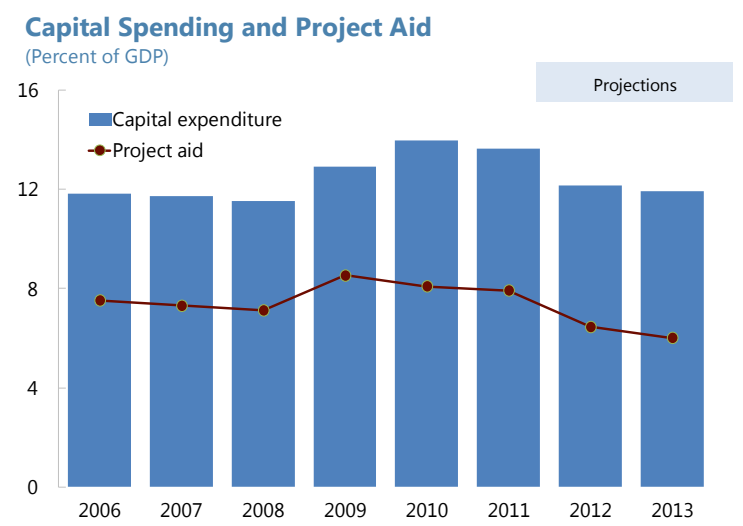

Net credit to government overshot its target slighted at end-2011, after overperforming significantly in the first half of the year.

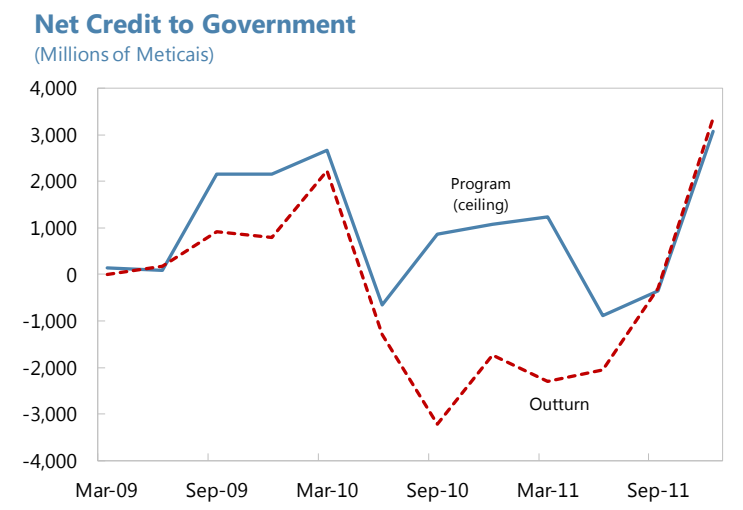

... while the rapid increase in spending has slowed down as a result of efforts to contain the wage bill and subsidies, especially the fuel subsidy.

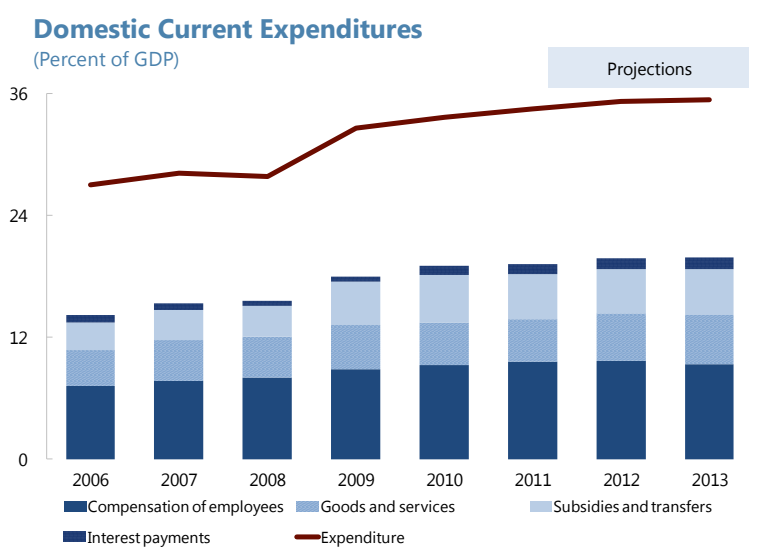

.... while strong revenue collections have offset the decline in external budget support, resulting in limited recourse to domestic financing.

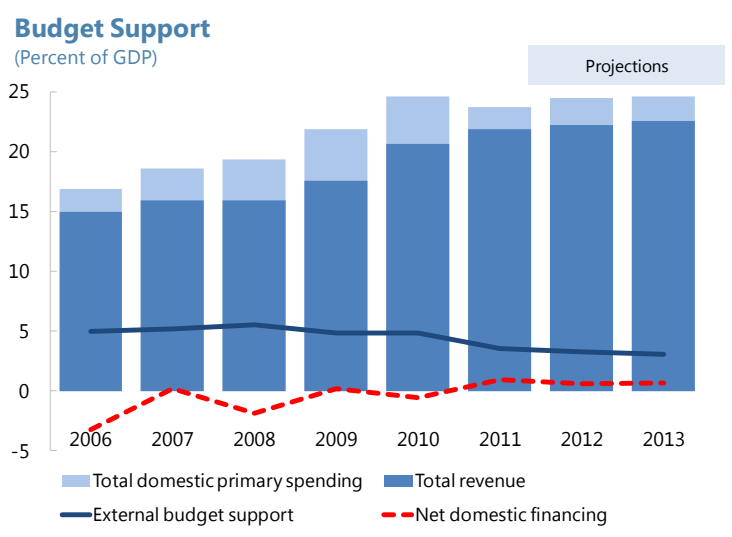

The overall fiscal stance was restrictive in 2011, thanks to strong revenue collections and restraint in current expenditure. Fiscal policy is set to become more accommodating in 2012.

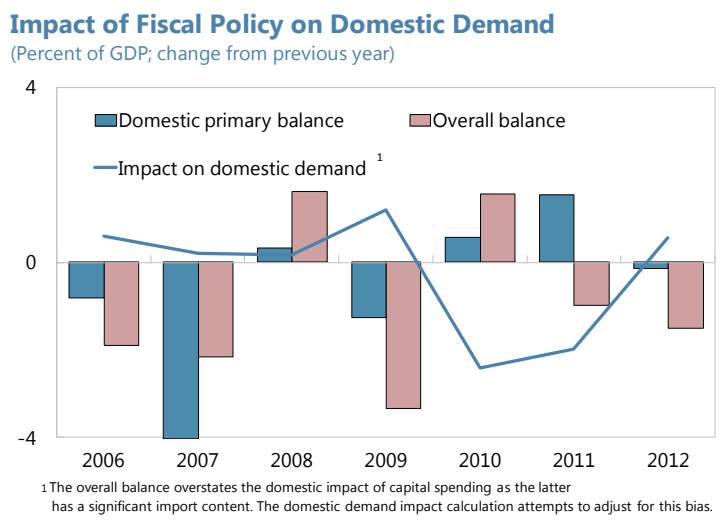

Sources: Mozambican authorities and IMF staff estimates and projections. 


\section{Monetary Policy and Liquidity Management}

\section{The BM's move toward a more} accommodating monetary policy stance in 2012 is appropriate. RM growth is projected to accelerate to about 20 percent, exceeding nominal GDP growth

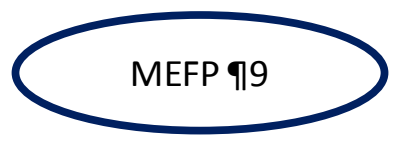
by one-fourth, thus supporting the provision of private sector credit at a critical time. To this effect, the BM has already eased key policy rates four times since last August, with a cumulative reduction of its lending facility rate and reserve requirements by 300 basis points and 75 basis points, respectively. The mission cautioned the BM that future policy decisions should continue to be guided by both the inflation outlook and credit conditions, taking into consideration the lags before the effects of policy changes become apparent.

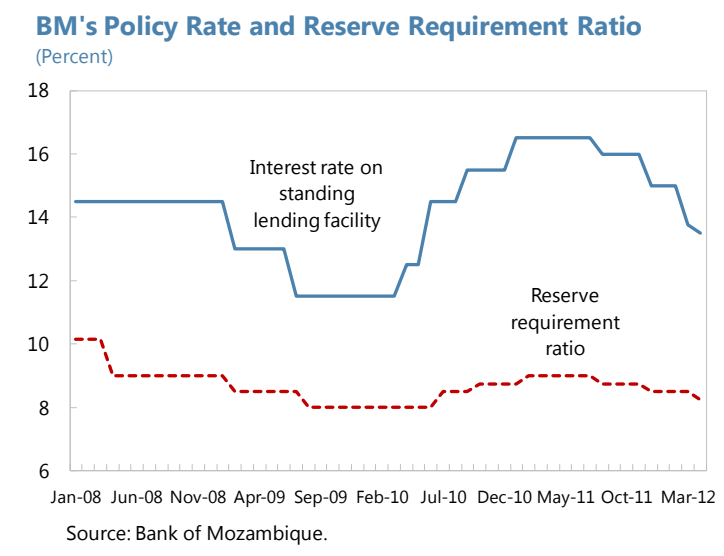

12. The $B M$ intends to continue enhancing the current framework for monetary policy operations and prepare for the adoption of an inflation-targeting (IT)

framework in the

medium term.

MEFP $\$ 10$

- Improving coordination with the government. The implementation of the Memorandum of Understanding on the exchange of information between the BM and the Ministry of Finance will help the $\mathrm{BM}$ in its liquidity forecasting.

- Developing monetary policy instruments and financial markets. Following Fund TA advice, the BM will further refine its monetary policy instruments. The authorities will also remove structural impediments to financial market development, including by harmonizing the taxation of treasury bills and government bonds, and also between collateralized and uncollateralized interbank market operations (structural benchmark).

- Preparing for an inflation-targeting (IT) framework. The BM will continue to improve data collection and analysis, forecasting modeling, and forward-looking communication, including by issuing its first quarterly monetary policy report to transparently explain its policy intentions (structural benchmark). 


\section{Mozambique's level of international} reserves remains comfortable. ${ }^{1}$ NIR growth

has been broadly in line with the growth of the economy, the deepening of the financial sector, and increased trade openness. The relatively higher reserve accumulation pace than in other countries of the region has

\section{Financial Sector Policy}

\section{Although the banking sector} remains resilient to the global financial turmoil, the BM will intensify its financial sector surveillance. System-wide indicators are relatively strong, while liquidity stress tests undertaken by the mission show that banks would be able to withstand a sizable funding shock on both domestic deposits and funding from domestic and foreign institutions. The assessment of the contagion risk stemming served the country

well to weather the MEFP $¥ 12$ impact of the global crisis and is consistent with ongoing structural changes in the Mozambican economy, which are likely to increase the required level of reserves over time.

from distressed foreign parent institutions, particularly the Portuguese banks which dominate the MEFP $\rrbracket 26$ market, suggests that domestic banks would have some buffers to cope with impaired deposits placed abroad. Nevertheless, the BM should, in response, intensify its financial sector surveillance (Box 1).

\footnotetext{
${ }^{1}$ See Appendix II on the assessment of reserve adequacy.
} 


\section{Box 1. Banking Vulnerabilities and Financial Sector Regulation ${ }^{1}$}

Although the banking sector has proved resilient to the crisis, the heightened risks call for strengthening risk monitoring, home-host cooperation, and crisis management arrangements.

The banking system's liquidity is large enough to withstand a sizable shock. Under a scenario with outflows in both deposits (20 percent) and funding from domestic and foreign institutions (30 percent) as shown in the table below, only two small banks would become illiquid.

\begin{tabular}{|lr|}
\hline Before Shock & (MT Billions) \\
Deposits & 130 \\
Assets & 195 \\
Shock & \\
Outflow of Funding [2] & $(29)$ \\
$\quad 20$ Percent of Deposits & $(26)$ \\
$\quad 30$ Percent of Wholesale Funding & $(3)$ \\
Cashable Assets [1] & 54 \\
After Shock & \\
Liquidity Buffer [1+2] & 25 \\
\hline
\end{tabular}

The BM considers implementing the following TA recommendations made during the fourth PSI review mission:

\section{- Further strengthening bank supervision. This} entails an analysis of funding risks, including the regular use of a liquidity stress testing framework based on highly frequent detailed data on banks' placements and funding (instrument, maturity, currency), as well as the surveillance of banks' placements abroad and deposit concentration. On the asset side, the non-performing loans (NPL) framework should align the definition and the recording of NPLs with international best standards so as to enhance transparency in the banking system. The authorities' legitimate concerns about cross-border risks are being further addressed through the drafting of a regulation that would introduce concentration limits on banks' placements abroad, including with related parties.
- Improving home-host cooperation. Homehost supervision is being strengthened through the signature of Memoranda of Understandings (MoU) with most home supervisors of foreignowned banks. The mission noted that the BM should also seek participation in supervisory colleges and the exchange of information in case of crisis management.

- Adjusting crisis management arrangements. The authorities' draft financial sector contingency plan is being adjusted by incorporating Fund TA recommendations, especially with respect to the modules on emergency liquidity assistance (ELA) and bank resolution. Specifically, an adequate legal framework for a resolution regime is needed for the authorities to effectively intervene into troubled banks. Furthermore, the ELA should apply not only to banks facing solvency pressures and considered as systemic institutions (as in the current financial sector contingency plan) but to any solvent bank facing a temporary liquidity problem. In addition, the Deposit Insurance Fund (DIF) created in 2010 is an integral component of the country's framework to safeguard financial sector stability, but its funding has yet to be secured and its deposit coverage to be defined. The mission suggested that the funds of the DIF could not only be used to compensate depositors in case of bank liquidation, but could also help recapitalize an insolvent bank and provide bank resolution through a bridge bank or a purchase and assumption (P\&A). The authorities expect to have the contingency plan fully operational by end-September 2012.

\footnotetext{
${ }^{1}$ This box updates and complements recent IMF analyses: See IMF Country Report No. 11/350 (Box 2) and IMF Country Report No. 11/149 (Section IV.C and Appendix IV).
} 


\section{B. Medium-Term Policies: Promoting Inclusive and Sustainable Growth}

15. Mozambique's medium term outlook remains favorable, but the government needs to close the infrastructure gap, maintain external and fiscal sustainability, and promote inclusive growth. The challenge is to cope with a trend decline in external aid, although revenues from the booming natural resource sector could help in the long run.

\section{Closing the Infrastructure Gap While Maintaining External Sustainability}

\section{There is a vast need for public} investment to rehabilitate and upgrade

Mozambique's infrastructure. Wide urban-

rural and interprovincial disparities remain despite the significant improvements made in recent years. When compared to an economy such as South Africa, inadequate infrastructure in Mozambique continues to represent a significant obstacle to improved living standards and economic productivity.

\section{On the other hand, Mozambique is} faced with a leveling off of donor support, which complicates investment plans. Net aid flows have already declined significantly from the global crisis-related peak of $141 / 2$ percent of GDP in 2009 to 12 percent of GDP in 2011, and are projected to level off to below 10 percent of GDP from 2012 onward. Furthermore, donors are moving to conditioning general budget support on performance, and shifting funds toward sector or project support, which may complicate the predictability of budget execution.

Mozambique: Total Public Investment Program, 2009-16

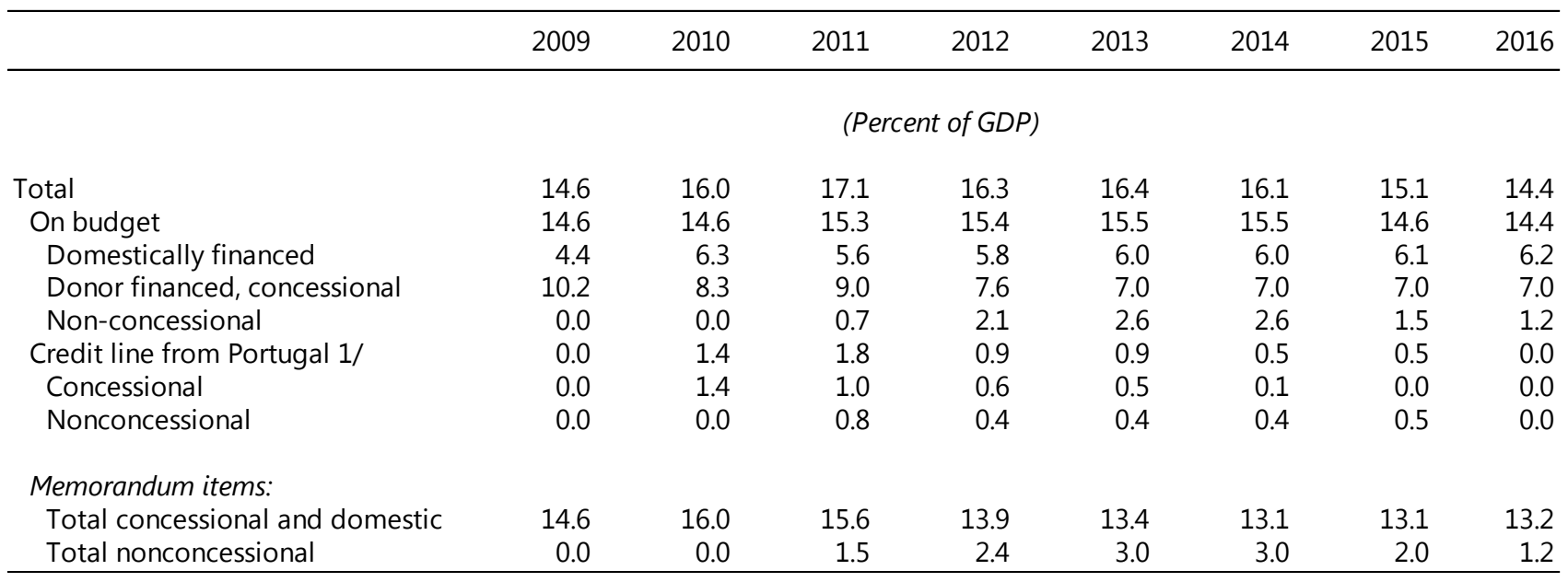

Source: IMF staff calculations.

1/ Portuguese credit line that will be implemented by the Road Fund. 


\section{Borrowing at nonconcessional terms}

\section{could help close the infrastructure gap. At}

the authorities' request, staff proposes an increase in the NCB ceiling to US\$1.5 billion, which would be consistent with absorptive capacity, debt sustainability, and

macroeconomic stability. Since introducing the current US\$900 million NCB limit under the

PSI, Mozambique's macroeconomic

performance and medium-term outlook have strengthened, and the pace of new loan disbursements has been slower-thanpreviously anticipated. These developments have improved debt indicators relative to the last DSA. ${ }^{2}$ In addition, the investment projects are unlikely to cause domestic demand pressures, as they typically contain a large import component and labor markets in Mozambique are underutilized. Moreover, the government has demonstrated in recent years that it can implement a large public investment program while maintaining macroeconomic stability. The proposed increase in the NCB ceiling would accommodate the $\$ 300$ million ring road loan which inadvertently turned out to be nonconcessional (see above), and would allow the authorities to undertake additional projects they consider most pressing. ${ }^{3}$ It would

\footnotetext{
${ }^{2}$ See the attached DSA update.

${ }^{3}$ Among other things, the government is close to contracting a commercial loan from China Eximbank
}

also preserve sufficient space for future borrowing for projects with an expected high economic return. ${ }^{4}$

\section{Informed borrowing and project} selection decisions would benefit from strong implementation of related reforms under the program.

Several reform steps MEFP $¥ 20$ have already been put in place and need to be used thoughtfully, including the recently adopted Integrated Investment Plan, the authorities' second own annual DSA, the newly created coordinating committees, and the finalized project selection templates and manuals. The reform process will further be helped by the implementation of new program commitments such as the forthcoming approval by the Council of Ministers of an enhanced medium-term debt strategy (MTDS), as well as the completion of a first annual domestic borrowing plan for 2013 (structural benchmark).

\section{Tapping the booming natural resource wealth could help the country}

for a toll bridge across Maputo Bay and a continuing road to South Africa, which was assessed by the World Bank. It is also considering loans for a hydroelectric power dam near Maputo and to supplement the rehabilitation of the Nacala airport.

\footnotetext{
${ }^{4}$ In particular, the authorities are working with several donors, including the World Bank, on a backbone power line from the Cahora-Bassa dam in central Mozambique to Maputo, which will entail a mix of private, concessional, and nonconcessional financing.
} 


\section{finance its priority expenditures in the long}

run. Mozambique is entering a period of rapid development of its mining and hydrocarbon sectors, with likely major macro-fiscal implications by the end of this decade. While coal production is gradually gaining steam in tandem with the expansion of transportation infrastructure, huge offshore natural gas discoveries are likely to lead to large-scale liquefied natural gas (LNG) production and exports beginning in 2018. This raises the prospect of large government revenues from this sector and could fuel spillover and industrial development in the whole economy.

\section{Reforms are underway to allow} Mozambique to take full advantage of the natural resource

boom. An April

2012 Fund TA

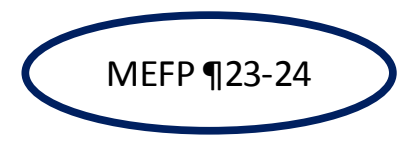

mission, under the Topical Trust Fund for

\section{Promoting Inclusive Growth}

\section{Despite sustained economic growth} and strides made toward the Millennium Development Goals, poverty reduction has

stalled. Staff analysis indicates that economic growth in Mozambique has not been as propoor as in countries with a similar growth performance and become less pro-poor over
Managing Natural Resource Wealth, recommended reforms to the mining and hydrocarbon fiscal regimes, which the authorities are currently considering, with a view to revising the relevant tax legislation this year. The government also plans to enhance its mining and hydrocarbon revenue forecasting, assess the feasibility of creating a stabilization fund to support the country's long-term development strategy, and improve the statistics on natural resources. Moreover, it is exploring opportunities for mutually beneficial renegotiations of terms under existing natural resources sector projects where changes in circumstances may have led to a misalignment. Finally, a key milestone in Mozambique's quest for full EITI membership was achieved by the completion of the second reconciliation country report in March 2012, which now needs to be assessed by the EITI Secretariat.

time. ${ }^{5}$ The poor are also extremely vulnerable to external shocks, as evidenced by the street riots over rising food prices in 2008 and 2010. The slow poverty reduction reflects structural rigidities and the narrow productive base of the economy, the low production and productivity in the agricultural sector where

\footnotetext{
${ }^{5}$ See Appendix III in IMF Country Report 11/149.
} 
three-fourths of the population earns its livelihood, and the slow progress in reforming the business environment to induce private sector activity; Mozambique ranked 139 out of 183 countries in the 2012 Doing Business Index, and 131 out of 139 countries in the 2010-11 World Economic Forum's Global Competitiveness Report. Finally, there has been little spillover of the megaprojects to the rest of the economy and to job creation.

\section{Against this backdrop, the} government's Poverty Reduction Strategy (PARP) for 2011-14 represents an important step towards reinvigorating the poverty reduction agenda. In the accompanying Joint Staff Advisory Note (Country Report No. 11/132), Bank and Fund staffs find that the PARP, with its three main and two supporting pillars, offers an appropriate path forward to sustain

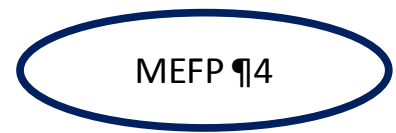
poverty reduction and make economic growth more inclusive. Its emphasis on broadening the productive and export base-especially its focus on agriculture-and on creating employment opportunities is conducive in this regard. Staffs also welcome the PARP's strong commitment to developing more focused and better-designed social protection programs in substitution of ad-hoc measures adopted in response to recent exogenous shocks.

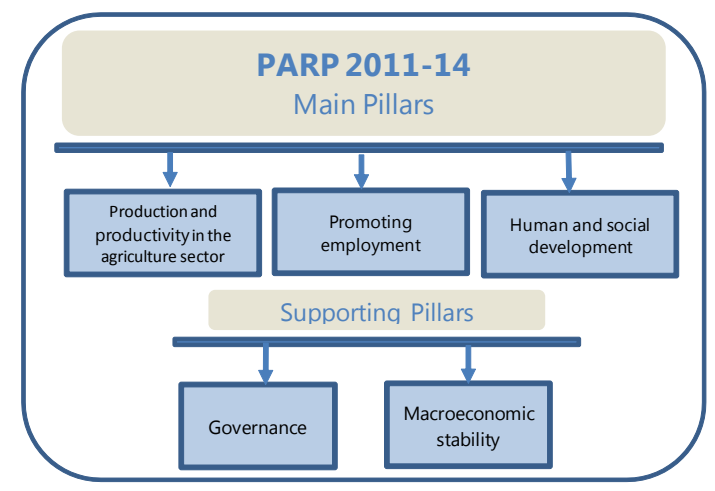

24. As advised in the JSAN, the authorities have taken on the implementation challenges and initiated measures in key areas. This includes reforms in the agricultural sector and some fast-track measures to improve the business environment in anticipation of the development of a new multi-year strategy later this year. In the social sector, a revamped social protection system was adopted last fall and is being implemented, supported by a model cooperation between the Fund, the World Bank, and ILO/UNICEF (Box 2). The mission welcomed the initial steps taken to implement the PARP. While recognizing the challenges of interministerial coordination, it encouraged the authorities to persevere in their efforts and consider a bold approach in order to induce a noticeable dent in poverty. 


\section{Box 2. Identifying Fiscal Space for a Social Protection Floor in Mozambique}

Social protection has gained a prominent position in the national debate in Mozambique. It is an important component of the PARP's third pillar on human and social development

As an exemplary case of collaboration, the Fund, the World Bank, and ILO/UNICEF joined forces in guiding the government in the design and implementation of a set of revised social protection programs. As a first step, the government revamped and streamlined its social protection systems last fall to comprise cash transfers, the provision of social services, and new programs for labor-intensive public works.

The Fund's role in the pilot was to identify the available fiscal space that could be allocated to a number of priorities over the medium term, including a social protection floor. On this basis, the government agreed to allocate adequate budget resources for social protection, which is also embedded in the authorities' economic program under the PSI.

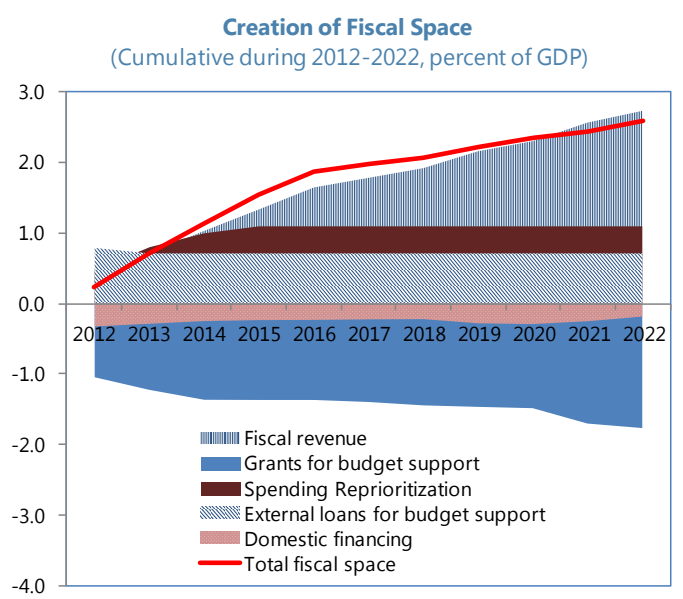

- Staff analysis suggests that additional fiscal space of $2 \frac{1}{2}$ percent of GDP could be created cumulatively during 2012-22, which could be allocated to all of the government's priority spending programs. Gains from continued reforms to revenue administration and the reprioritization of expenditure through the phasing out the fuel subsidy would be the driving forces in the process, which would more than offset the trend decline in foreign aid.
- The analysis suggests that the government's current target of reaching 815,000 poor households would require an annual resource allocation of 0.4-0.8 percent of GDP over the next few years, which would take advantage of part of the newly created fiscal space. This is consistent with a sustainable medium-term fiscal framework and macroeconomic stability. However, reaching this target would require successful capacity building, as outlined below.

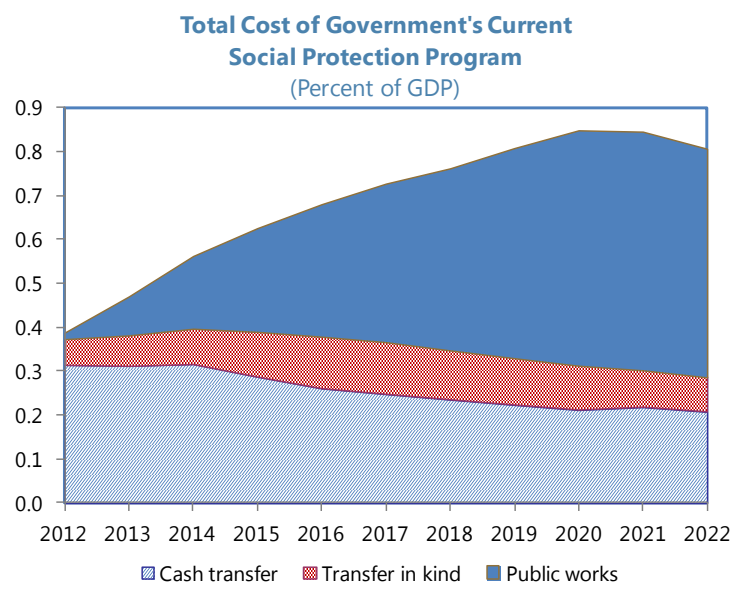

- Using a larger share of the identified fiscal space for social protection could be considered in the longer term, if the government chooses to do so. However, social protection would need to compete with other government priorities, such as infrastructure investment.

- The fiscal space for overall priority expenditure could be larger than the projected $2 \frac{1}{2} 2$ percent of GDP if more resources become available, such as from the natural resource sector and donors.

\section{Going forward, the implementation challenges} are enormous. With the support of the World Bank and ILO/UNICEF, the government aims to enhance its targeting and beneficiary identification systems, payment procedures, and monitoring systems. It will also seek to build capacity at the district level and will assess the possibility of obtaining further donor resources, possibly through the creation of a common fund which is currently being discussed. 


\section{Deepening Structural Reforms}

\section{Deepening structural reforms is} crucial in supporting implementation of the

PARP's five pillars and in ensuring

sustained economic growth and

macroeconomic stability. Many of the

structural reforms embedded in the

authorities' economic program under the PSI-

including as structural conditionality-will

specifically support achievement of the PARP's

supporting pillar on maintaining a stable

macroeconomic environment, such as the

reforms in the area of tax administration and

policy, public financial management, and the fiscal and monetary policy frameworks. Other reforms target the PARP's job creation pillar by supporting private sector activity, such as the MEFP १4,10-11,19-29

reforms aimed at financial sector stability and development and the business climate. Finally, the governance pillar is being promoted by reforms related to the anti-corruption and AML/CFT frameworks.

Structural Conditionality, 2012-13

\begin{tabular}{|c|c|c|}
\hline Structural Benchmarks & $\begin{array}{l}\text { Expected Date of } \\
\text { Implementation }\end{array}$ & Macro relevance \\
\hline $\begin{array}{l}\text { The Bank of Mozambique will publish its first quarterly monetary policy report, as } \\
\text { described in paragraph } 11 \text { of the MEFP dated November 14, } 2011 .\end{array}$ & End-May 2012 & $\begin{array}{l}\text { Enhance transparency and accountability of monetary policy, } \\
\text { thereby better anchoring price expectations and facilitating the } \\
\text { transition to inflation targeting. }\end{array}$ \\
\hline $\begin{array}{l}\text { The Government will complete the rollout of the e-Folha electronic salary payment } \\
\text { system to all provincial directorates, as described in paragraph } 19 \text { of the MEFP } \\
\text { dated November } 14,2011 \text {. }\end{array}$ & End-June 2012 & $\begin{array}{l}\text { Improve budget planning and execution, and enhance the } \\
\text { authorities' handle on the wage bill. }\end{array}$ \\
\hline $\begin{array}{l}\text { The Ministry of Finance will make fully functional a new single taxpayer database } \\
\text { and identification number, as described in paragraph } 23 \text { of the MEFP dated } \\
\text { November } 14,2011 \text {. }\end{array}$ & End-June 2012 & $\begin{array}{l}\text { Help create fiscal space and shore up the authorities' revenue } \\
\text { objectives. }\end{array}$ \\
\hline $\begin{array}{l}\text { The Government will submit to Parliament an amendment to the Corporate Income } \\
\text { Tax Law reflecting measures derived from an impact study on the equalization of } \\
\text { the tax treatment between Treasury bills and Government bonds and between } \\
\text { collateralized and uncollateralized interbank market operations, to take effect in } \\
2013 \text {, as described in paragraph } 10 \text { of the MEFP dated November } 14,2011 \text {. }\end{array}$ & End-September 2012 & $\begin{array}{l}\text { Enhance the effectiveness of money market operations, develop } \\
\text { domestic financial markets and improve the liquidity of } \\
\text { government securities. }\end{array}$ \\
\hline $\begin{array}{l}\text { The Minister of Finance will approve a time-bound action plan for IPSAS- } \\
\text { compatible reporting, as described in paragraph } 19 \text { of this MEFP. }\end{array}$ & End-October 2012 & Improve the reporting and monitoring of budget execution. \\
\hline $\begin{array}{l}\text { The Government will adopt an Annual Borrowing Plan for 2013, as described in } \\
\text { paragraph } 20 \text { of this MEFP. }\end{array}$ & End-January 2013 & $\begin{array}{l}\text { Improve debt management through better internal planning and } \\
\text { communication with the market, thereby reducing issuance cost. }\end{array}$ \\
\hline
\end{tabular}




\section{PROGRAM ISSUES}

\section{Modifications are proposed for} several assessment criteria (ACs) and indicative targets. The end-June 2012 ACs on reserve money and net international reserves and the indicative floor on government revenues were adjusted according to performance to date and the authorities' short-term policy objectives to implement a countercyclical policy stance that would help shield Mozambique from the effect of the global downturn. The AC on nonconcessional external borrowing was raised based on Mozambique's infrastructure needs, consistent with absorptive capacity, debt sustainability, and macroeconomic stability. Finally, the indicative target on priority spending has been redefined to incorporate outlays related to social action, labor and employment.

buffers to pursue a countercyclical policy stance this year and next to support economic growth. This entails allowing automatic stabilizers to function to keep spending at budgeted levels and pursuing an accommodative monetary policy stance to support the provision of credit to the economy at a crucial moment in time. However, the authorities are encouraged to remain vigilant about the re-emergence of inflation pressures that could compromise the recent disinflation gains. In addition, the authorities' intention to step up financial sector supervision is crucial to guard against potential cross-border contagion.

\section{Nonetheless, the global downturn is} likely to have repercussions on economic activity going forward. Mozambique's prudent track record of macroeconomic policies provides the authorities with ample

\section{The government should vigorously} implement its poverty reduction strategy. The PARP represents an adequate framework to reinvigorate the recent stagnation in 
poverty reduction which appears to be at odds with Mozambique's stellar economic growth record. The implementation challenges are enormous, such as with respect to the necessary inter-ministerial coordination and the development of bold sectoral strategies. Nonetheless, the first steps taken in implementing the PARP are encouraging, including in particular the fast-track measures to improve the business environment and the progress made in getting a revamped social protection system off the ground. On the other hand, the authorities are urged to prevent the costly fuel subsidy from reemerging and eroding valuable fiscal space for priority spending. They should persevere in their efforts and work with civil society and development partners in the PARP's implementation.

\section{Prudent borrowing and project} selection decisions will be essential in addressing Mozambique's infrastructure

gap. While infrastructure investment is conducive to generating sustained and inclusive economic growth, the government should build on its ongoing debt management and project selection reforms to make informed decisions and preserve the country's comfortable debt indicators. Faced with a trend decline in donor support, it should be mindful of the risks entailed in an overly aggressive use of nonconcessional borrowing. The proposed increase in the program's nonconcessional borrowing ceiling-consistent with absorptive capacity, macroeconomic stability, and debt sustainability-aims to strike a balance between addressing pressing infrastructure bottlenecks with preserving sufficient space for future borrowing for projects with an expected high economic return.

\section{In the long run, the natural resource} sector is best placed to help the government afford its infrastructure needs. In this context, staff commends the authorities for their intention to move rapidly in modernizing the fiscal regimes for the booming mining and emerging hydrocarbon sectors and the planned institutional enhancements to manage natural resource wealth, and encourages them to continue their efforts to get full EITI membership.

\section{Based on program performance and} the authorities' strong ownership of the Fund-supported program, staff recommends completion of the fourth review under the PSI. Staff also recommends a waiver for the minor nonobservance of the end-December AC on net credit to the government and the modification of ACs. 
Table 1. Mozambique: Selected Economic and Financial Indicators, 2009-17

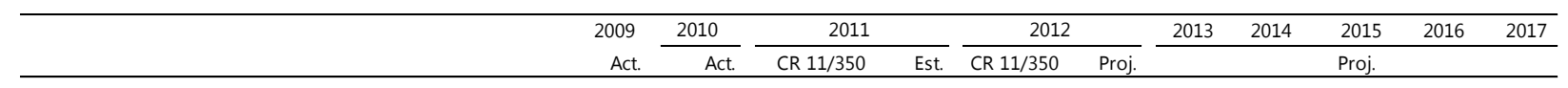

(Annual percentage change, unless otherwise indicated)

National income and prices

Nominal GDP (MT billion)

Nominal GDP growth

Real GDP growth

GDP per capita (US\$)

Consumer price index (annual average)

Consumer price index (end of period)

Exchange rate, MT per US dollar, eop

Exchange rate, MT per US dollar, per.avg

External sector

Merchandise exports

Merchandise exports, excluding megaprojects

Merchandise imports

Merchandise imports, excluding megaprojects

Terms of trade

Nominal effective exchange rate (end of period)

Real effective exchange rate (end of period)

$\begin{array}{rrrrrrrrrrr}266 & 313 & 372 & 371 & 430 & 426 & 484 & 553 & 632 & 721 & 823 \\ 10.8 & 17.5 & 18.9 & 18.6 & 15.6 & 14.9 & 13.6 & 14.2 & 14.3 & 14.1 & 14.1 \\ 6.3 & 6.8 & 7.2 & 7.1 & 7.5 & 6.7 & 7.2 & 7.8 & 7.8 & 7.8 & 7.8 \\ 471 & 439 & 578 & 580 & 607 & 632 & 675 & 741 & 814 & 893 & 978 \\ 3.3 & 12.7 & 10.8 & 10.4 & 7.2 & 7.2 & 5.6 & 5.6 & 5.6 & 5.6 & 5.6 \\ 4.2 & 16.6 & 8.4 & 5.5 & 5.6 & 5.6 & 5.6 & 5.7 & 5.5 & 5.6 & 5.6 \\ 27.5 & 32.8 & \ldots & 27.1 & \ldots & \ldots & \ldots & \ldots & \ldots & \ldots & \ldots \\ 26.7 & 33.0 & \ldots & 29.1 & \ldots & \ldots & \ldots & \ldots & \ldots & \ldots & \ldots \\ & & & & & & & & & & \\ -19.1 & 8.7 & 13.5 & 19.0 & 12.2 & 12.3 & 14.2 & 11.9 & 11.0 & 9.4 & 9.3 \\ 4.3 & -20.5 & 8.1 & 14.4 & 3.1 & 4.0 & 4.0 & 4.4 & 5.5 & 5.5 & 6.0 \\ -6.1 & 2.6 & 14.7 & 19.2 & 5.6 & 6.5 & 9.6 & 8.6 & 7.6 & 7.3 & 7.3 \\ -10.6 & -0.7 & 6.2 & 1.0 & 6.6 & 11.9 & 8.8 & 8.0 & 6.0 & 7.5 & 8.4 \\ -0.7 & 14.4 & 7.0 & 18.1 & 9.5 & 13.3 & 9.5 & 7.8 & 4.0 & 0.2 & -1.5 \\ -7.1 & -22.4 & \ldots . & 12.4 & \ldots & \ldots & \ldots & \ldots & \ldots & \ldots & \ldots \\ -6.5 & -15.1 & \ldots & 19.8 & \ldots & \ldots & \ldots & \ldots & \ldots & \ldots & \ldots\end{array}$

(Annual percentage change, unless otherwise indicated)

$\begin{array}{lllllllllll}27.3 & 29.2 & 13.2 & 8.5 & 18.3 & 20.2 & 18.1 & 19.8 & 20.3 & 21.3 & 21.9 \\ 32.6 & 22.8 & 11.7 & 8.1 & 18.9 & 20.2 & 18.6 & 19.5 & 19.5 & 19.6 & 21.1\end{array}$

$\begin{array}{lllllllllll}58.6 & 29.3 & 17.4 & 6.3 & 21.8 & 22.8 & 23.2 & 23.5 & 23.7 & 23.9 & 24.1\end{array}$

$\begin{array}{lllllllllll}26.8 & 29.5 & 28.7 & 26.5 & 30.3 & 28.3 & 30.7 & 33.2 & 35.9 & 39.0 & 42.4\end{array}$

(Percent of GDP)

Investment and saving

Gross domestic investment

Government

Other sectors

Gross domestic savings (excluding grants)

Government

Other sectors

External current account, before grants

External current account, after grants

$\begin{array}{lllllllllll}16.5 & 22.0 & 21.2 & 24.3 & 22.0 & 22.8 & 24.3 & 24.7 & 24.1 & 24.3 & 24.5\end{array}$

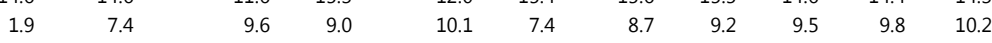

$\begin{array}{lllllllllll}-2.6 & 3.9 & 4.3 & 5.5 & 6.1 & 5.5 & 7.4 & 8.5 & 8.6 & 9.3 & 10.0\end{array}$

$\begin{array}{lllllllllll}-0.4 & 1.6 & 1.6 & 2.7 & 2.6 & 2.4 & 2.7 & 2.8 & 2.9 & 3.1 & 3.3\end{array}$

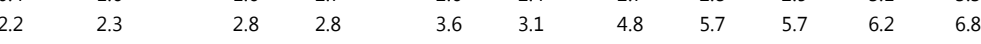

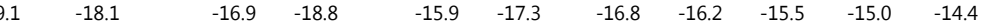

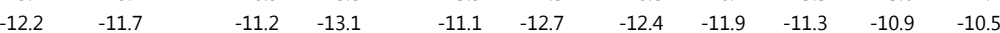

Government budget

Total expenditure and net lending

Overall balance, before grants

Total grants

Overall balance, after grants

Domestic primary balance, before grants

External financing (incl. debt relief)

Net domestic financing

Privatization

Total public debt ${ }^{1}$

Of which: external

Of which: domestic

PV of PPG external debt

\begin{tabular}{rrrrrrrrrrr}
17.6 & 20.6 & 21.4 & 21.9 & 22.2 & 22.2 & 22.6 & 22.9 & 23.2 & 23.5 & 23.6 \\
32.6 & 33.6 & 32.6 & 34.5 & 34.6 & 35.2 & 35.5 & 35.6 & 34.9 & 34.9 & 34.7 \\
-15.0 & -13.0 & -11.3 & -12.7 & -12.4 & -13.0 & -12.9 & -12.7 & -11.7 & -11.4 & -11.1 \\
9.5 & 9.1 & 7.7 & 7.7 & 6.5 & 6.6 & 6.3 & 6.1 & 6.1 & 6.1 & 6.1 \\
-5.5 & -4.0 & -3.5 & -4.9 & -5.9 & -6.4 & -6.6 & -6.6 & -5.6 & -5.3 & -4.9 \\
-4.3 & -3.7 & -3.1 & -2.2 & -2.1 & -2.3 & -2.1 & -1.8 & -1.8 & -1.7 & -1.7 \\
5.1 & 4.2 & 2.8 & 4.3 & 5.3 & 5.8 & 5.9 & 5.9 & 5.0 & 4.6 & 4.2 \\
0.2 & -0.4 & 0.8 & 0.9 & 0.5 & 0.6 & 0.6 & 0.7 & 0.7 & 0.7 & 0.7 \\
0.2 & 0.0 & 0.0 & 0.0 & 0.0 & 0.0 & 0.0 & 0.0 & 0.0 & 0.0 & 0.0 \\
40.5 & 36.8 & 31.7 & 34.4 & 37.6 & 41.4 & 43.9 & 46.6 & 47.0 & 47.0 & 46.6 \\
37.3 & 34.5 & 29.7 & 31.5 & 35.2 & 38.3 & 40.6 & 43.0 & 43.1 & 43.0 & 42.4 \\
3.2 & 2.4 & 2.0 & 2.9 & 2.4 & 3.1 & 3.4 & 3.6 & 3.8 & 4.0 & 4.2 \\
$\ldots$ & 18.7 & $\ldots$ & 17.5 & $\ldots$ & 22.5 & 25.1 & 27.6 & 28.3 & 28.6 & 28.5 \\
& & (Millions of U.S. dollars, unless otherwise indicated) & & & & \\
$-1,908$ & $-1,718$ & $-2,149$ & $-2,401$ & $-2,166$ & $-2,463$ & $-2,602$ & $-2,800$ & $-3,008$ & $-3,257$ & $-3,502$ \\
$-1,220$ & $-1,113$ & $-1,422$ & $-1,667$ & $-1,508$ & $-1,802$ & $-1,917$ & $-2,068$ & $-2,187$ & $-2,367$ & $-2,544$ \\
194 & 58 & 122 & 331 & 251 & 200 & 281 & 398 & 409 & 424 & 533 \\
1,840 & 1,908 & 2,030 & 2,238 & 2,280 & 2,438 & 2,719 & 3,117 & 3,526 & 3,950 & 4,482 \\
2,012 & 2,099 & 2,227 & 2,429 & 2,474 & 2,626 & 2,905 & 3,299 & 3,673 & 4,060 & 4,557 \\
5.2 & 4.4 & 4.5 & 4.8 & 4.6 & 4.7 & 4.8 & 5.0 & 5.1 & 5.2 & 5.3 \\
\hline
\end{tabular}

Sources: Mozambican authorities; and IMF staff estimates and projections.

${ }^{1}$ Consistent with DSA definition, the nonconcessional Portuguese credit line is included under the external debt

${ }^{2}$ Includes disbursements of IMF resources under the ESF and August 2009 SDR allocation

External current account, after grants

Overall balance of payments

Net international reserves (end of period) ${ }^{2}$

Gross international reserves (end of period) ${ }^{2}$

Months of projected imports of goods and nonfactor services 
Table 2. Mozambique: Government Finances, 2009-12

(Billions of Meticais)

\begin{tabular}{|c|c|c|c|c|c|c|}
\hline & \multirow{2}{*}{$\begin{array}{c}2009 \\
\text { Act. }\end{array}$} & \multirow{2}{*}{$\begin{array}{c}2010 \\
\text { Est. }\end{array}$} & \multicolumn{2}{|c|}{2011} & \multicolumn{2}{|c|}{2012} \\
\hline & & & CR $11 / 350$ & Est. & CR $11 / 350$ & Proj. \\
\hline Total revenue & 46.73 & 64.48 & 79.46 & 81.12 & 95.54 & 94.57 \\
\hline Tax revenue & 41.47 & 56.54 & 69.94 & 71.54 & 83.85 & 82.90 \\
\hline Income and profits & 13.72 & 18.48 & 22.69 & 24.89 & 27.85 & 27.49 \\
\hline Goods and services & 22.89 & 31.69 & 38.85 & 38.42 & 46.26 & 45.76 \\
\hline International trade & 4.08 & 5.26 & 6.73 & 6.73 & 8.08 & 8.00 \\
\hline Other & 0.78 & 1.10 & 1.67 & 1.51 & 1.66 & 1.66 \\
\hline Nontax revenue & 5.26 & 7.94 & 9.51 & 9.58 & 11.69 & 11.66 \\
\hline Total expenditure and net lending & 86.70 & 105.20 & 121.41 & 128.26 & 148.91 & 150.09 \\
\hline Current expenditure & 47.87 & 59.56 & 73.65 & 71.50 & 84.46 & 84.46 \\
\hline Compensation to employees & 23.62 & 29.11 & 36.25 & 35.66 & 41.35 & 41.35 \\
\hline Goods and services & 11.72 & 13.09 & 16.27 & 15.57 & 19.84 & 19.84 \\
\hline Of which: expenditure on ENSSB & & 0.73 & 0.75 & 0.75 & 1.01 & 1.01 \\
\hline Interest on public debt & 1.36 & 2.67 & 3.31 & 3.58 & 4.63 & 4.63 \\
\hline Transfer payments & 11.17 & 14.69 & 17.82 & 16.46 & 18.63 & 18.63 \\
\hline Of which: fuel subsidy (old stock) & 2.80 & 4.69 & 3.59 & 4.09 & 2.68 & 2.68 \\
\hline Domestic current primary balance & 0.22 & 7.59 & 9.12 & 13.21 & 15.71 & 14.74 \\
\hline Capital expenditure & 34.41 & 43.70 & 43.00 & 50.60 & 51.58 & 51.74 \\
\hline Domestically financed & 11.72 & 20.03 & 20.58 & 20.32 & 24.26 & 24.26 \\
\hline Externally financed & 22.69 & 23.67 & 22.42 & 30.28 & 27.31 & 27.48 \\
\hline Net lending & 4.42 & 1.93 & 4.76 & 6.17 & 12.88 & 13.89 \\
\hline Domestically financed & -0.02 & -0.40 & 0.19 & 0.39 & 0.40 & 0.40 \\
\hline Externally financed loans to public enterprises & 4.44 & 2.33 & 4.56 & 5.77 & 12.48 & 13.49 \\
\hline of which: nonconcessional net lending & 0.00 & 0.00 & 1.46 & 2.51 & 7.62 & 8.77 \\
\hline Unallocated revenue $(+) /$ expenditure $(-)^{1}$ & 0.00 & 0.36 & 0.00 & -0.84 & 0.00 & 0.00 \\
\hline Domestic primary balance, before grants, above the line ${ }^{2}$ & -11.47 & -11.68 & -11.66 & -8.34 & -8.95 & -9.92 \\
\hline Overall balance, before grants & -39.97 & -40.36 & -41.95 & -47.98 & -53.38 & -55.52 \\
\hline Grants received & 25.30 & 28.34 & 28.82 & 28.63 & 28.11 & 28.11 \\
\hline Project support & 16.18 & 17.11 & 16.51 & 16.37 & 18.31 & 18.48 \\
\hline Direct financing & 0.37 & 0.00 & 0.00 & 0.00 & 0.00 & 0.00 \\
\hline Budget support & 9.12 & 11.24 & 12.31 & 12.26 & 9.80 & 9.63 \\
\hline Overall balance, after grants & -14.67 & -12.01 & -13.13 & -19.35 & -25.26 & -27.41 \\
\hline Net external financing & 13.50 & 13.25 & 10.32 & 15.99 & 22.91 & 24.91 \\
\hline Disbursements & 14.20 & 14.28 & 11.36 & 17.11 & 24.91 & 27.19 \\
\hline Project & 6.51 & 8.19 & 5.91 & 13.01 & 9.00 & 9.00 \\
\hline Nonproject support & 7.69 & 6.09 & 5.45 & 4.10 & 15.91 & 18.18 \\
\hline Loans to public enterprises & 3.89 & 2.33 & 4.56 & 5.77 & 12.35 & 13.87 \\
\hline Budget support & 3.80 & 3.76 & 0.89 & 0.83 & 3.56 & 4.31 \\
\hline Cash amortization & -0.70 & -1.03 & -1.04 & -1.12 & -2.00 & -2.27 \\
\hline Net domestic financing & 0.52 & -1.22 & 2.90 & 3.36 & 2.35 & 2.50 \\
\hline Net privatization & 0.41 & -0.01 & -0.09 & 0.00 & 0.00 & 0.00 \\
\hline \multicolumn{7}{|l|}{ Memorandum items: } \\
\hline Net aid flows & 38.26 & 40.78 & 36.60 & 43.63 & 42.33 & 42.80 \\
\hline Gross aid flows & 39.50 & 42.62 & 38.71 & 45.74 & 45.40 & 46.15 \\
\hline Budget support & 12.92 & 14.99 & 13.19 & 13.09 & 13.36 & 13.95 \\
\hline Nonbudget support & 26.58 & 27.63 & 25.52 & 32.65 & 32.04 & 32.21 \\
\hline Project support & 22.69 & 25.30 & 22.42 & 29.39 & 27.31 & 27.48 \\
\hline Loans to public enterprises & 3.89 & 2.33 & 3.10 & 3.26 & 4.73 & 4.73 \\
\hline External debt service & 1.24 & 1.85 & 2.11 & 2.11 & 3.07 & 3.35 \\
\hline
\end{tabular}

Sources: Mozambican authorities; and IMF staff estimates and projections.

${ }^{1}$ Residual discrepancy between identified sources and uses of funds.

${ }^{2}$ Revenue minus noninterest current expenditure minus locally financed capital expenditure and locally financed net lending. 
Table 3. Mozambique: Government Finances, 2009-17

(Percent of GDP)

\begin{tabular}{|c|c|c|c|c|c|c|c|c|c|c|c|}
\hline & 2009 & 2010 & $20]$ & & 201 & & 2013 & 2014 & 2015 & 2016 & 2017 \\
\hline & Act. & Est. & $\begin{array}{c}\text { CR } \\
11 / 350 \\
\end{array}$ & Est. & $\begin{array}{c}C R \\
11 / 350 \\
\end{array}$ & Proj. & & & jections & & \\
\hline Total revenue & 17.6 & 20.6 & 21.4 & 21.9 & 22.2 & 22.2 & 22.6 & 22.9 & 23.2 & 23.5 & 23.6 \\
\hline Tax revenue & 15.6 & 18.1 & 18.8 & 19.3 & 19.5 & 19.5 & 19.8 & 20.2 & 20.5 & 20.8 & 20.9 \\
\hline Nontax revenue & 2.0 & 2.5 & 2.6 & 2.6 & 2.7 & 2.7 & 2.7 & 2.7 & 2.7 & 2.7 & 2.7 \\
\hline Total expenditure and net lending & 32.6 & 33.6 & 32.6 & 34.6 & 34.6 & 35.2 & 35.5 & 35.6 & 34.9 & 34.9 & 34.7 \\
\hline Current expenditure & 18.0 & 19.0 & 19.8 & 19.3 & 19.6 & 19.8 & 19.9 & 20.1 & 20.3 & 20.4 & 20.4 \\
\hline Compensation to employees & 8.9 & 9.3 & 9.7 & 9.6 & 9.6 & 9.7 & 9.4 & 9.1 & 8.8 & 8.9 & 8.8 \\
\hline Goods and services & 4.4 & 4.2 & 4.4 & 4.2 & 4.6 & 4.7 & 4.9 & 5.0 & 5.2 & 5.3 & 5.3 \\
\hline Interest on public debt & 0.5 & 0.9 & 0.9 & 1.0 & 1.1 & 1.1 & 1.2 & 1.3 & 1.4 & 1.5 & 1.5 \\
\hline Transfer payments & 4.2 & 4.7 & 4.8 & 4.4 & 4.3 & 4.4 & 4.5 & 4.8 & 4.8 & 4.8 & 4.8 \\
\hline Of which: fuel subsidy (old stock) & 1.1 & 1.5 & 1.0 & 1.1 & 0.6 & 0.6 & $\ldots$ & $\ldots$ & $\ldots$ & $\ldots$ & $\ldots$ \\
\hline new voucher programs & $\ldots$ & $\ldots$ & 0.1 & 0.1 & 0.3 & 0.3 & $\ldots$ & $\ldots$ & $\ldots$ & $\ldots$ & $\ldots$ \\
\hline Capital expenditure & 12.9 & 14.0 & 11.6 & 13.6 & 12.0 & 12.1 & 11.9 & 11.9 & 12.1 & 12.1 & 12.4 \\
\hline Domestically financed & 4.4 & 6.4 & 5.5 & 5.5 & 5.6 & 5.7 & 5.9 & 5.9 & 6.0 & 6.1 & 6.4 \\
\hline Externally financed & 8.5 & 7.6 & 6.0 & 8.2 & 6.3 & 6.4 & 6.0 & 6.0 & 6.0 & 6.0 & 6.0 \\
\hline Net lending & 1.7 & 0.6 & 1.3 & 1.7 & 3.0 & 3.3 & 3.6 & 3.6 & 2.5 & 2.3 & 1.9 \\
\hline Domestically financed & 0.0 & -0.1 & 0.1 & 0.1 & 0.1 & 0.1 & 0.1 & 0.1 & 0.1 & 0.1 & 0.1 \\
\hline Externally financed loans to public enterprises & 1.7 & 0.7 & 1.2 & 1.6 & 2.9 & 3.2 & 3.6 & 3.5 & 2.4 & 2.2 & 1.8 \\
\hline of which: concessional & 1.7 & 0.7 & 0.8 & 0.9 & 1.1 & 1.1 & 1.0 & 1.0 & 1.0 & 1.0 & 1.0 \\
\hline of which: nonconcessional net lending & 0.0 & 0.0 & 0.4 & 0.7 & 1.8 & 2.1 & 2.6 & 2.6 & 1.5 & 1.2 & 0.8 \\
\hline Unallocated revenue $(+) /$ expenditure $(-) 1 /$ & 0.0 & 0.1 & 0.0 & -0.2 & 0.0 & 0.0 & 0.0 & 0.0 & 0.0 & 0.0 & 0.0 \\
\hline Domestic primary balance, before grants 2/ & -4.3 & -3.7 & -3.1 & -2.2 & -2.1 & -2.3 & -2.1 & -1.8 & -1.8 & -1.7 & -1.7 \\
\hline Overall balance, before grants & -15.0 & -12.9 & -11.3 & -12.9 & -12.4 & -13.0 & -12.9 & -12.7 & -11.7 & -11.4 & -11.1 \\
\hline Grants received & 9.5 & 9.1 & 7.7 & 7.7 & 6.5 & 6.6 & 6.3 & 6.1 & 6.1 & 6.1 & 6.1 \\
\hline Project & 6.1 & 5.5 & 4.4 & 4.4 & 4.3 & 4.3 & 4.3 & 4.2 & 4.2 & 4.1 & 4.2 \\
\hline Investment projects & 2.7 & 2.3 & 2.1 & 1.8 & 2.0 & 2.0 & 2.0 & 2.0 & 2.0 & 2.1 & 2.3 \\
\hline Special programs & 3.2 & 3.2 & 2.4 & 2.6 & 2.2 & 2.3 & 2.2 & 2.2 & 2.2 & 2.0 & 1.9 \\
\hline Direct financing & 0.1 & 0.0 & 0.0 & 0.0 & 0.0 & 0.0 & 0.0 & 0.0 & 0.0 & 0.0 & 0.0 \\
\hline Nonproject & 3.4 & 3.6 & 3.3 & 3.3 & 2.3 & 2.3 & 2.1 & 1.9 & 1.9 & 1.9 & 1.9 \\
\hline Overall balance, after grants & -5.5 & -4.0 & -3.5 & -5.2 & -5.9 & -6.4 & -6.6 & -6.6 & -5.6 & -5.3 & -4.9 \\
\hline Net external financing & 5.1 & 4.2 & 2.8 & 4.3 & 5.3 & 5.8 & 5.9 & 5.9 & 5.0 & 4.6 & 4.2 \\
\hline Disbursements & 5.3 & 4.6 & 3.1 & 4.6 & 5.8 & 6.4 & 6.5 & 6.7 & 5.9 & 5.8 & 5.5 \\
\hline Project & 2.4 & 2.6 & 1.6 & 3.5 & 2.1 & 2.1 & 1.8 & 1.8 & 1.9 & 1.9 & 1.8 \\
\hline Nonproject support & 2.9 & 1.9 & 1.5 & 1.1 & 3.7 & 4.3 & 4.7 & 4.9 & 4.0 & 3.9 & 3.6 \\
\hline Loans to public enterprises & 1.5 & 0.7 & 1.2 & 1.6 & 2.9 & 3.3 & 3.8 & 4.0 & 3.1 & 3.0 & 2.7 \\
\hline of which: nonconcessional & 0.0 & 0.0 & 0.4 & 0.7 & 1.8 & 2.1 & 2.8 & 3.0 & 2.1 & 1.9 & 1.7 \\
\hline Budget support & 1.4 & 1.2 & 0.2 & 0.2 & 0.8 & 1.0 & 0.9 & 0.9 & 0.9 & 0.9 & 0.9 \\
\hline Cash amortization & -0.3 & -0.3 & -0.3 & -0.3 & -0.5 & -0.5 & -0.5 & -0.8 & -0.9 & -1.2 & -1.2 \\
\hline Net domestic financing & 0.2 & -0.4 & 0.8 & 0.9 & 0.5 & 0.6 & 0.6 & 0.7 & 0.7 & 0.7 & 0.7 \\
\hline Net privatization & 0.2 & 0.0 & 0.0 & 0.0 & 0.0 & 0.0 & 0.0 & 0.0 & 0.0 & 0.0 & 0.0 \\
\hline Memorandum items: & & & & & & & & & & & \\
\hline Net aid flows & 14.5 & 13.0 & 9.8 & 11.8 & 9.8 & 10.0 & 9.1 & 8.6 & 8.4 & 8.1 & 8.0 \\
\hline Gross aid flows & 15.0 & 13.6 & 10.4 & 12.3 & 10.6 & 10.8 & 10.0 & 9.9 & 9.9 & 9.9 & 9.9 \\
\hline Budget support & 4.9 & 4.8 & 3.5 & 3.5 & 3.1 & 3.3 & 3.0 & 2.9 & 2.9 & 2.9 & 2.8 \\
\hline Nonbudget support & 10.1 & 8.8 & 6.9 & 8.8 & 7.4 & 7.6 & 7.0 & 7.0 & 7.0 & 7.0 & 7.0 \\
\hline Project support & 8.6 & 8.1 & 6.0 & 7.9 & 6.3 & 6.4 & 6.0 & 6.0 & 6.0 & 6.0 & 6.0 \\
\hline Loans to public enterprises & 1.5 & 0.7 & 0.8 & 0.9 & 1.1 & 1.1 & 1.0 & 1.0 & 1.0 & 1.0 & 1.0 \\
\hline External debt service & 0.5 & 0.6 & 0.6 & 0.6 & 0.7 & 0.8 & 0.9 & 1.3 & 1.5 & 1.8 & 1.9 \\
\hline
\end{tabular}

Sources: Mozambican authorities; and IMF staff estimates and projections.

${ }^{1}$ Residual discrepancy between identified sources and uses of funds.

${ }^{2}$ Revenue minus noninterest current expenditure minus locally financed capital expenditure and locally financed net lending. 
Table 4. Mozambique: Monetary Survey, Quarterly, 2009-12

(Billions of Meticais, unless otherwise specified)

\begin{tabular}{|c|c|c|c|c|c|c|c|c|c|c|}
\hline & \multirow{3}{*}{$\begin{array}{r}2009 \\
\text { Act. }\end{array}$} & \multirow{3}{*}{$\frac{2010}{\text { Act. }}$} & \multicolumn{4}{|c|}{2011} & \multicolumn{4}{|c|}{2012} \\
\hline & & & \multicolumn{2}{|c|}{ Q3 } & \multicolumn{2}{|c|}{ Q4 } & \multirow{2}{*}{$\begin{array}{r}\mathrm{Q} 1 \\
\mathrm{CR} / 11 / 350\end{array}$} & \multirow{2}{*}{$\begin{array}{l}\text { Q2 } \\
\text { Proj. }\end{array}$} & \multirow{2}{*}{$\begin{array}{l}\text { Q3 } \\
\text { Proj. }\end{array}$} & \multirow{2}{*}{$\begin{array}{l}\text { Q4 } \\
\text { Proj }\end{array}$} \\
\hline & & & Prog. & Act. & $\mathrm{CR} / 11 / 350$ & Act. & & & & \\
\hline \multicolumn{11}{|l|}{ Bank of Mozambique } \\
\hline Net foreign assets & 49.6 & 56.9 & 54.4 & 53.7 & 54.2 & 56.9 & 57.4 & 62.8 & 67.9 & 73.0 \\
\hline (US\$ billions) & 1.7 & 1.7 & 1.7 & 2.0 & 1.9 & 2.1 & 1.9 & 2.1 & 2.2 & 2.3 \\
\hline Net international reserves & 53.7 & 62.2 & 59.5 & 57.9 & 58.9 & 61.1 & 62.2 & 67.3 & 72.6 & 78.0 \\
\hline (US\$ billions) & 1.8 & 1.9 & 1.9 & 2.1 & 2.0 & 2.2 & 2.1 & 2.2 & 2.3 & 2.4 \\
\hline Net domestic assets & -25.2 & -25.2 & -20.2 & -22.0 & -18.4 & -22.6 & -24.0 & -26.0 & -30.5 & -31.8 \\
\hline Credit to government (net) & -25.3 & -20.6 & -20.2 & -30.3 & -23.7 & -26.8 & -19.2 & -27.7 & -25.8 & -24.3 \\
\hline Credit to banks (net) & 2.3 & 0.4 & 2.4 & 1.7 & 4.8 & -3.8 & 2.0 & 0.4 & -4.5 & -5.0 \\
\hline Credit to the economy & 0.4 & 0.5 & 0.5 & 0.8 & 0.6 & 0.9 & 0.6 & 0.9 & 0.9 & 0.9 \\
\hline Other items (net; assets +) & -2.6 & -5.6 & -2.8 & 5.8 & -0.1 & 7.2 & -7.5 & 0.5 & -1.1 & -3.3 \\
\hline Reserve money & 24.5 & 31.6 & 34.2 & 31.8 & 35.8 & 34.3 & 33.4 & 36.8 & 37.4 & 41.2 \\
\hline Currency in circulation & 16.1 & 20.4 & 22.5 & 20.1 & 23.6 & 21.9 & 21.2 & 23.4 & 24.2 & 27.6 \\
\hline Currency outside banks & 13.1 & 17.4 & 20.6 & 17.0 & 11.3 & 17.5 & 10.8 & 20.2 & 20.1 & 21.9 \\
\hline Currency in Banks (Cash in Vault) & 3.1 & 3.1 & 1.9 & 3.1 & 12.3 & 4.4 & 10.4 & 3.2 & 4.1 & 5.7 \\
\hline Bank deposits in BM & 8.3 & 11.2 & 11.7 & 11.7 & 12.1 & 12.4 & 12.2 & 13.3 & 13.2 & 13.6 \\
\hline \multicolumn{11}{|l|}{ Commercial Banks } \\
\hline Net foreign assets & 14.1 & 22.8 & 11.7 & 13.3 & 16.8 & 16.1 & 24.0 & 28.9 & 29.8 & 25.2 \\
\hline (Millions of U.S. dollars) & 0.5 & 0.7 & 0.4 & 0.5 & 0.6 & 0.6 & 0.8 & 0.9 & 1.0 & 0.8 \\
\hline Net domestic assets & 79.3 & 92.5 & 118.4 & 102.6 & 118.7 & 109.1 & 111.7 & 116.7 & 113.8 & 123.7 \\
\hline Banks' reserves & 11.7 & 14.3 & 13.6 & 15.1 & 24.5 & 16.8 & 22.6 & 16.6 & 17.3 & 19.3 \\
\hline Credit to BM (net) & -2.5 & -0.3 & -2.4 & -0.6 & -4.8 & 4.0 & -2.0 & -0.4 & 4.5 & 5.0 \\
\hline Credit to government (net) & 13.8 & 8.4 & 7.3 & 20.9 & 14.2 & 21.1 & 12.9 & 20.1 & 20.3 & 21.9 \\
\hline Credit to the economy & 71.0 & 91.9 & 108.6 & 94.1 & 106.3 & 97.3 & 106.6 & 120.9 & 119.4 & 119.7 \\
\hline Other items (net; assets +) & -14.8 & -21.8 & -8.6 & -27.0 & -21.4 & -30.1 & -28.4 & -40.5 & -47.6 & -42.2 \\
\hline Deposits & 93.4 & 115.3 & 130.1 & 115.9 & 135.5 & 125.3 & 135.7 & 145.7 & 143.6 & 148.9 \\
\hline Demand and savings deposits & 60.2 & 73.0 & 81.9 & 73.1 & 89.6 & 79.1 & 88.3 & 90.0 & 89.7 & 93.5 \\
\hline Time deposits & 33.2 & 42.3 & 48.2 & 42.8 & 45.9 & 46.2 & 47.4 & 55.6 & 54.0 & 55.4 \\
\hline \multicolumn{11}{|l|}{ Monetary Survey } \\
\hline Net foreign assets & 63.7 & 79.6 & 66.1 & 67.0 & 71.0 & 73.0 & 81.4 & 91.7 & 97.8 & 98.2 \\
\hline (US\$ billions) & 2.2 & 2.4 & 2.1 & 2.5 & 2.4 & 2.7 & 2.7 & 3.0 & 3.1 & 3.1 \\
\hline Net domestic assets & 43.3 & 51.8 & 84.6 & 65.9 & 75.9 & 69.1 & 65.1 & 74.2 & 66.0 & 72.6 \\
\hline Domestic credit & 60.0 & 80.2 & 96.1 & 85.6 & 97.3 & 92.4 & 100.9 & 114.1 & 114.7 & 118.1 \\
\hline Credit to government (net) & -11.5 & -12.2 & -13.0 & -9.3 & -9.5 & -5.7 & -6.3 & -7.6 & -5.6 & -2.5 \\
\hline Credit to the economy & 71.4 & 92.4 & 109.1 & 94.9 & 106.9 & 98.2 & 107.3 & 121.7 & 120.2 & 120.6 \\
\hline Cred. economy in foreign currency & 22.8 & -21.8 & 33.6 & 22.0 & 24.6 & 21.8 & 24.5 & 25.6 & 24.6 & 26.6 \\
\hline Other items (net; assets + ) & -16.6 & -28.3 & -11.5 & -19.6 & -21.5 & -23.4 & -35.9 & -39.9 & -48.7 & -45.5 \\
\hline Money and quasi money (M3) & 107.1 & 131.5 & 150.7 & 132.9 & 146.8 & 142.1 & 146.4 & 165.9 & 163.8 & 170.8 \\
\hline Foreign currency deposits & 34.5 & 46.2 & 55.6 & 37.1 & 46.9 & 38.1 & 47.0 & 44.6 & 42.7 & 42.0 \\
\hline (US\$ billions) & 1.2 & 1.4 & 1.8 & 1.4 & 1.6 & 1.4 & 1.6 & 1.5 & 1.4 & 1.3 \\
\hline M2 & 72.5 & 85.3 & 95.1 & 95.9 & 99.9 & 104.0 & 99.4 & 121.3 & 121.0 & 128.8 \\
\hline Memorandum Items & & & & & & & & & & \\
\hline Avg daily reserve money in 3rd month of quarter & 23.6 & 31.8 & 33.7 & 31.4 & 36.1 & 33.3 & 33.8 & 36.9 & 37.0 & 40.0 \\
\hline 12-month percent change & 28.5 & 35.0 & 16.6 & 8.8 & 13.2 & 4.5 & 16.6 & 20.1 & 17.8 & 20.2 \\
\hline Avg daily currency in 3rd month of quarter & 15.7 & 20.4 & 21.9 & 19.8 & 23.6 & 21.2 & 21.5 & 23.0 & 23.9 & 26.7 \\
\hline 12-month percent change & 39.4 & 30.1 & 22.6 & 10.8 & 15.7 & 3.7 & 24.1 & 22.8 & 20.7 & 26.0 \\
\hline NCG stock (prog def.) ${ }^{1}$ & -14.6 & -15.8 & -16.7 & -15.9 & -13.5 & -12.5 & -10.7 & -11.6 & -9.7 & -6.5 \\
\hline NCG flow (prog def.) cum from end-year ${ }^{1}$ & 0.5 & -1.2 & -0.4 & -0.1 & 2.9 & 3.4 & 2.8 & -2.6 & -0.7 & 2.5 \\
\hline 12 -month percent change & & & & & & & & & & \\
\hline Reserve money & 27.3 & 29.2 & 16.6 & 8.2 & 13.2 & 8.5 & 16.6 & 20.1 & 17.8 & 20.2 \\
\hline M2 & 34.6 & 17.6 & 19.7 & 20.6 & 17.2 & 21.9 & 18.3 & 33.8 & 26.2 & 23.8 \\
\hline M3 & 32.6 & 22.8 & 17.8 & 3.9 & 11.7 & 8.1 & 13.2 & 27.8 & 23.2 & 20.2 \\
\hline Credit to the economy & 58.6 & 29.3 & 19.6 & 4.1 & 17.4 & 6.3 & 16.1 & 28.9 & 26.7 & 22.8 \\
\hline Money multiplier ( $\mathrm{M} 2 /$ reserve money) & 2.97 & 2.70 & 2.78 & 3.02 & 2.79 & 3.03 & 2.98 & 3.30 & 3.23 & 3.12 \\
\hline Velocity (GDP/M2) & 3.67 & 3.67 & $\ldots$ & $\ldots$ & 3.72 & 3.57 & $\ldots$ & 3.51 & 3.52 & 3.31 \\
\hline Nominal GDP & 266 & 313 & $\ldots$ & $\ldots$ & 372 & 371 & $\ldots$ & $\ldots$ & $\ldots$ & 426 \\
\hline Nominal GDP growth & 10.8 & 17.5 & $\ldots$ & $\ldots$ & 18.9 & 18.6 & $\ldots$ & $\ldots$ & $\ldots$ & 14.9 \\
\hline Policy lending rate (end-of-period) & 11.5 & 15.5 & $\ldots$ & 16.0 & $\ldots$ & 15.0 & $\ldots$ & $\ldots$ & $\ldots$ & $\ldots$ \\
\hline T-bill 91 days rate & 9.5 & 14.7 & $\ldots$ & 14.3 & $\ldots$ & 11.8 & $\ldots$ & $\ldots$ & $\ldots$ & $\ldots$ \\
\hline
\end{tabular}

Sources: Bank of Mozambique; and IMF staff estimates and projections

${ }^{1}$ As defined in the TMU (excluding the non concessional Portuguese credit line). 
Table 5. Mozambique: Balance of Payments, 2009-17

(Millions of U.S. dollars, unless otherwise specified)

\begin{tabular}{|c|c|c|c|c|c|c|c|c|c|c|c|}
\hline & \multirow{2}{*}{$\begin{array}{r}2009 \\
\text { Act. }\end{array}$} & 2010 & \multicolumn{2}{|c|}{2011} & \multicolumn{2}{|c|}{2012} & 2013 & 2014 & 2015 & 2016 & 2017 \\
\hline & & Est. & CR $11 / 350$ & Est. & CR $11 / 350$ & Proj. & \multicolumn{5}{|c|}{ Projections } \\
\hline Current account balance & $-1,220$ & $-1,113$ & $-1,422$ & $-1,667$ & $-1,508$ & $-1,803$ & $-1,918$ & $-2,069$ & $-2,188$ & $-2,367$ & $-2,545$ \\
\hline Trade balance for goods & $-1,275$ & $-1,179$ & $-1,380$ & $-1,411$ & $-1,283$ & $-1,344$ & $-1,329$ & $-1,325$ & $-1,288$ & $-1,288$ & $-1,284$ \\
\hline Of which: Megaprojects & 520 & 768 & 677 & 468 & 934 & 817 & 1,061 & 1,285 & 1,483 & 1,710 & 1,987 \\
\hline Exports, f.o.b. & 2,147 & 2,333 & 2,649 & 2,776 & 2,972 & 3,116 & 3,558 & 3,982 & 4,420 & 4,835 & 5,286 \\
\hline Of which: Megaproject exports & 1,311 & 1,668 & 1,931 & 2,015 & 2,231 & 2,325 & 2,734 & 3,122 & 3,513 & 3,879 & 4,272 \\
\hline Imports, f.o.b. & $-3,422$ & $-3,512$ & $-4,029$ & $-4,187$ & $-4,255$ & $-4,460$ & $-4,887$ & $-5,306$ & $-5,708$ & $-6,123$ & $-6,570$ \\
\hline Of which: Megaproject imports & -791 & -900 & $-1,254$ & $-1,547$ & $-1,297$ & $-1,508$ & $-1,673$ & $-1,837$ & $-2,030$ & $-2,169$ & $-2,285$ \\
\hline Trade balance for services & -457 & -506 & -855 & -796 & -865 & -895 & -973 & $-1,089$ & $-1,220$ & $-1,385$ & $-1,558$ \\
\hline Income balance & -251 & -85 & -29 & -207 & -142 & -239 & -316 & -403 & -520 & -605 & -685 \\
\hline Of which: Dividend payments by megaprojects & -17 & 0 & -5 & -157 & -169 & -254 & -310 & -375 & -466 & -539 & -614 \\
\hline Current transfers balance & 763 & 657 & 842 & 746 & 783 & 675 & 700 & 748 & 840 & 911 & 981 \\
\hline Of which: External grants & 687 & 605 & 726 & 734 & 659 & 661 & 685 & 731 & 821 & 891 & 958 \\
\hline Capital and financial account balance & 1,288 & 1,113 & 1,544 & 2,270 & 1,759 & 2,005 & 2,199 & 2,467 & 2,597 & 2,791 & 3,077 \\
\hline Capital account balance & 422 & 346 & 372 & 379 & 398 & 422 & 474 & 531 & 585 & 676 & 805 \\
\hline Financial account balance & 865 & 768 & 1,172 & 1,891 & 1,361 & 1,583 & 1,725 & 1,936 & 2,013 & 2,115 & 2,272 \\
\hline Net foreign direct investment & 890 & 790 & 1,009 & 2,090 & 1,018 & 1,590 & 1,575 & 1,749 & 1,890 & 1,912 & 1,976 \\
\hline Net foreign borrowing by the general government & 434 & 468 & 590 & 593 & 923 & 926 & 1,024 & 1,087 & 980 & 913 & 939 \\
\hline Net foreign borrowing by the nonfinancial private sector & -487 & -348 & -304 & -610 & -303 & -671 & -629 & -626 & -581 & -540 & -477 \\
\hline Other $^{1}$ & 28 & -142 & -122 & -182 & -278 & -261 & -245 & -274 & -277 & -170 & -165 \\
\hline Net errors and omissions & 126 & 58 & 0 & -272 & 0 & 0 & 0 & 0 & 0 & 0 & 0 \\
\hline Overall balance & 194 & 58 & 122 & 331 & 251 & 200 & 281 & 398 & 409 & 424 & 533 \\
\hline External financing & -194 & -58 & -122 & -331 & -251 & -200 & -281 & -398 & -409 & -424 & -533 \\
\hline Reserve assets ${ }^{1}$ & -352 & -87 & -128 & -329 & -248 & -198 & -278 & -395 & -374 & -387 & -497 \\
\hline Net use of Fund credit & 156 & 18 & 6 & -2 & -3 & -2 & -3 & -3 & -36 & -37 & -36 \\
\hline Exceptional financing & 2 & 10 & 0 & 0 & 0 & 0 & 0 & 0 & 0 & 0 & 0 \\
\hline \multicolumn{12}{|l|}{ Memorandum items: } \\
\hline \multicolumn{12}{|l|}{ Effective exchange rate indexes (percent change) } \\
\hline Nominal effective exchange rate & -7.1 & -22.4 & $\ldots$ & $\ldots$ & $\ldots$ & $\ldots$ & $\ldots$ & $\ldots$ & $\ldots$ & $\ldots$ & ... \\
\hline Real effective exchange rate & -6.5 & -15.1 & $\ldots$ & $\ldots$ & $\ldots$ & $\ldots$ & $\ldots$ & $\ldots$ & $\ldots$ & $\ldots$ & $\ldots$ \\
\hline Terms of trade index (percent change) & -0.7 & 14.4 & 7.0 & 18.1 & 9.5 & 13.3 & 9.5 & 7.8 & 4.0 & 0.2 & -1.5 \\
\hline International aluminum price & -35.3 & 30.2 & 14.3 & 10.5 & -1.0 & -6.4 & 5.2 & 3.7 & 2.7 & 2.1 & 2.0 \\
\hline International food price index & -14.7 & 11.5 & 22.1 & 19.7 & -4.4 & -7.5 & -3.1 & -3.5 & -4.8 & -1.5 & -1.0 \\
\hline International fuel price index & -36.9 & 26.0 & 29.5 & 31.5 & -3.5 & 9.2 & -4.1 & -5.7 & -4.7 & -3.3 & -2.1 \\
\hline International coal price index & $\ldots$ & $\ldots$ & 22.8 & 23.2 & -14.1 & -12.3 & -17.2 & -2.3 & -2.2 & 0.0 & 0.0 \\
\hline Current account balance (percent of GDP) & -12.2 & -11.7 & -11.2 & -13.1 & -11.1 & -12.7 & -12.4 & -11.9 & -11.3 & -10.9 & -10.5 \\
\hline Excluding external grants & -19.1 & -18.1 & -16.9 & -18.8 & -15.9 & -17.4 & -16.8 & -16.2 & -15.5 & -15.0 & -14.4 \\
\hline Gross aid inflows (percent of GDP) & 15.8 & 13.9 & 11.8 & 11.1 & 11.8 & 11.3 & 10.6 & 10.1 & 10.0 & 10.0 & 10.0 \\
\hline Of which: To central government & 15.0 & 13.6 & 10.4 & 12.3 & 10.6 & 10.8 & 10.0 & 9.9 & 9.9 & 9.9 & 9.9 \\
\hline Budget support & 4.9 & 4.8 & 3.5 & 3.5 & 3.1 & 3.3 & 3.0 & 2.9 & 2.9 & $2.9^{\prime \prime}$ & 2.8 \\
\hline Nonbudge support & 10.1 & 8.8 & 6.9 & 8.8 & 7.4 & 7.6 & 7.0 & 7.0 & 7.0 & 7.0 & 7.0 \\
\hline Project support & 8.6 & 8.1 & 6.0 & 7.9 & 6.3 & 6.4 & 6.0 & 6.0 & 6.0 & 6.0 & 6.0 \\
\hline Onlending & 1.5 & 0.7 & 0.8 & 0.9 & 1.1 & 1.1 & 1.0 & 1.0 & 1.0 & 1.0 & 1.0 \\
\hline Net foreign assets & 1,701 & 1,745 & 1,857 & 2,083 & 2,108 & 2,285 & 2,566 & 2,964 & 3,373 & 3,797 & 4,330 \\
\hline Net international reserves ${ }^{1}$ & 1,840 & 1,908 & 2,030 & 2,238 & 2,280 & 2,438 & 2,719 & 3,117 & 3,526 & 3,950 & 4,483 \\
\hline Gross international reserves ${ }^{1}$ & 2,012 & 2,099 & 2,227 & 2,429 & 2,474 & 2,627 & 2,905 & 3,299 & 3,673 & 4,060 & 4,557 \\
\hline Months of projected imports of goods and nonfactor services & 5.2 & 4.4 & 4.5 & 4.8 & 4.6 & 4.7 & 4.8 & 5.0 & 5.1 & 5.2 & 5.3 \\
\hline Months of current imports of goods and nonfactor services & 5.4 & 5.4 & 4.7 & 5.2 & 5.0 & 5.2 & 5.3 & 5.4 & 5.6 & 5.7 & 5.9 \\
\hline
\end{tabular}

Sources: Data from Government of Mozambique and projections by IMF staff.

${ }^{1}$ Accounts for disbursement of an SDR allocation of SDR 108.8 million in $2009 \mathrm{Q} 3$ above the line under other investment liabilities of the monetary authorities, and below the line as an increase in reserve assets. 
Table 6. Mozambique: Financial Soundness Indicators for Banking Sector, 2001-11

\begin{tabular}{|c|c|c|c|c|c|c|c|c|c|c|c|c|c|c|}
\hline & 2001 & 2002 & 2003 & 2004 & 2005 & 2006 & 2007 & 2008 & 2009 & 2010 & Mar-11 & Jun-11 & Sep-11 & Dec-11 \\
\hline \multicolumn{15}{|l|}{ Capital adequacy } \\
\hline Regulatory capital to risk-weighted assets & 5.5 & 14.0 & 16.5 & 18.0 & 13.4 & 12.5 & 14.2 & 13.9 & 15.1 & 14.4 & 15.3 & 18.7 & 17.8 & 17.1 \\
\hline Regulatory Tier I capital to risk-weighted assets & 6.0 & 12.0 & 14.7 & 16.0 & 13.6 & 10.7 & 12.1 & 12.4 & 13.0 & 13.7 & 13.8 & 16.8 & 16.5 & 16.1 \\
\hline Capital (net worth) to assets & 8.2 & 9.4 & 7.4 & 7.4 & 6.6 & 6.3 & 7.2 & 7.5 & 7.7 & 8.0 & 8.5 & 10.8 & 9.8 & 9.0 \\
\hline \multicolumn{15}{|l|}{ Asset composition and quality } \\
\hline Foreign exchange loans to total loans & 64.7 & 69.9 & 60.8 & 62.0 & 51.4 & 33.2 & 28.5 & 32.8 & 32.4 & 29.5 & 27.2 & 25.0 & 25.5 & 25.1 \\
\hline Nonperforming loans to gross loans $1 /$ & 23.4 & 22.0 & 13.8 & 5.9 & 3.5 & 3.1 & 2.6 & 1.9 & 1.8 & 1.9 & 2.3 & 2.3 & 2.4 & 2.6 \\
\hline Nonperforming loans net of provisions to capital 1 / & 11.0 & 9.4 & 8.8 & 3.8 & 1.9 & 3.6 & 0.5 & 2.5 & 5.9 & 5.6 & 6.1 & 5.9 & 6.1 & 6.6 \\
\hline \multicolumn{15}{|l|}{ Earnings and profitability } \\
\hline Return on assets & 0.1 & 1.6 & 1.4 & 1.5 & 1.9 & 4.0 & 3.8 & 3.5 & 3.0 & 2.6 & 2.9 & 3.0 & 2.6 & 2.5 \\
\hline Return on equity & 3.5 & 22.1 & 18.6 & 20.6 & 26.9 & 60.8 & 50.7 & 44.7 & 36.6 & 32.9 & 36.0 & 34.6 & 28.8 & 26.5 \\
\hline Interest margin to gross income & 10.2 & 61.4 & 62.1 & 65.8 & 63.6 & 67.4 & 70.2 & 58.8 & 55.7 & 59.4 & 64.4 & 66.8 & 66.1 & 64.9 \\
\hline Noninterest expenses to gross income & 16.9 & 67.0 & 81.9 & 81.6 & 75.2 & 60.2 & 60.8 & 58.7 & 58.4 & 59.7 & 61.4 & 58.2 & 60.1 & 61.3 \\
\hline Personnel expenses to noninterest expenses & 51.7 & 44.7 & 42.4 & 43.1 & 43.5 & 42.6 & 46.3 & 45.1 & 45.9 & 45.5 & 46.7 & 47.1 & 47.7 & 47.1 \\
\hline Trading and fee income to gross income & 33.1 & 39.7 & 37.9 & 34.2 & 36.4 & 32.6 & 29.5 & 40.5 & 44.3 & 23.8 & 18.3 & 16.0 & 16.3 & 17.2 \\
\hline Spread between reference loan and deposit rates (90 days, local currency) & 14.0 & 19.0 & 17.4 & 14.9 & 13.8 & 14.6 & 11.1 & 10.7 & 11.1 & 10.3 & 9.6 & 12.0 & 12.0 & $\ldots$ \\
\hline \multicolumn{15}{|l|}{ Funding and liquidity } \\
\hline Liquid assets to total assets $2 /$ & 34.6 & 53.9 & 45.2 & 38.3 & 31.1 & 33.9 & 36.0 & 36.2 & 27.9 & 22.4 & 22.1 & 23.2 & 24.9 & 27.8 \\
\hline Customer deposits to total (non-interbank) loans & 217.0 & 240.0 & 193.6 & 205.0 & 177.6 & 169.5 & 184.9 & 165.7 & 138.2 & 131.2 & 129.6 & 126.9 & 124.6 & 131.6 \\
\hline
\end{tabular}

Source: Bank of Mozambique (BM).

Nonperforming loans are defined according to Mozambican accounting standards (they include only part of the past-due loans).

2/ Includes deposits at parent banks. 
Table 7. Mozambique: Quantitative Assessment Criteria and Indicative Targets ${ }^{1}$

(Millions of meticais, unless otherwise specified)

\begin{tabular}{|c|c|c|c|c|c|c|c|c|c|c|c|c|c|}
\hline & \multicolumn{7}{|c|}{2011} & \multirow{4}{*}{ Status } & \multicolumn{5}{|c|}{2012} \\
\hline & \multirow{2}{*}{\multicolumn{3}{|c|}{$\begin{array}{c}\text { End-Sept } \\
\text { Indicative Target }\end{array}$}} & \multirow{2}{*}{\multicolumn{4}{|c|}{$\begin{array}{c}\text { End-Dec } \\
\text { Assessment Criteria }\end{array}$}} & & \multirow{3}{*}{$\begin{array}{r}\begin{array}{c}\text { End-March } \\
\text { Indicative } \\
\text { Target }\end{array} \\
\text { Prog. }\end{array}$} & \multirow{2}{*}{\multicolumn{2}{|c|}{$\begin{array}{l}\text { End-June } \\
\text { Assessment } \\
\text { Criteria }\end{array}$}} & \multirow{3}{*}{$\begin{array}{c}\begin{array}{c}\text { End-Sept } \\
\text { Indicative } \\
\text { Target }\end{array} \\
\text { Proposed } \\
\end{array}$} & \multirow{3}{*}{$\begin{array}{c}\text { End-Dec } \\
\text { Assessment } \\
\text { Criteria } \\
\text { Proposed }\end{array}$} \\
\hline & & & & & & & & & & & & & \\
\hline & Prog. & Adj. & Act. & & Prog. & Adj. & Act. & & & Prog. & Proposed & & \\
\hline \multicolumn{14}{|l|}{ Assessment Criteria for end-June/December } \\
\hline Net credit to the government (cumulative ceiling) & -362 & -362 & -74 & NM & 2,896 & 3,066 & 3,360 & NM & 2,795 & $-2,645$ & $-2,645$ & -745 & 2,500 \\
\hline Stock of reserve money (ceiling) & 33,708 & 33,400 & 31,436 & M & 36,053 & 36,053 & 33,275 & M & 33,800 & 36,734 & 36,923 & 37,031 & 39,985 \\
\hline Stock of net international reserves of the BM (floor, US\$ millions) & 1,905 & 1,905 & 2,130 & M & 2,030 & 2,038 & 2,238 & M & 2,091 & 2,154 & 2,207 & 2,323 & 2,438 \\
\hline $\begin{array}{l}\text { New nonconcessional external debt contracted or guaranteed by the central } \\
\text { government or the BM or selected state-owned enterprises with maturity of one year } \\
\text { or more (cumulative ceiling over the duration of the program, US\$ millions) }\end{array}$ & 900 & 900 & 146 & M & 900 & 900 & 146 & M & 900 & 900 & 1,500 & 1,500 & 1,500 \\
\hline Stock of short-term external public debt outstanding (ceiling) & 0 & 0 & 0 & M & 0 & 0 & 0 & M & 0 & 0 & 0 & 0 & 0 \\
\hline External payments arrears (ceiling, US\$ millions) & 0 & 0 & 0 & M & 0 & 0 & 0 & M & 0 & 0 & 0 & 0 & 0 \\
\hline \multicolumn{14}{|l|}{ Indicative targets } \\
\hline Government revenue (cumulative floor) & 56,382 & 56,382 & 60,490 & M & 76,792 & 76,792 & 81,119 & M & 20,272 & 45,556 & 45,256 & 69,687 & 94,568 \\
\hline Priority spending (cumulative floor) & 40,839 & 40,839 & 40,589 & NM & 58,000 & 58,000 & 58,242 & M & 10,500 & 24,500 & 24,500 & 35,934 & 72,563 \\
\hline
\end{tabular}


Table 8. Mozambique: Structural Conditionality under the current PSI, 2011-12

\begin{tabular}{|c|c|c|c|}
\hline Structural Benchmarks & $\begin{array}{c}\text { Expected } \\
\text { Implementation }\end{array}$ & Status & Macroeconomic importance \\
\hline $\begin{array}{l}\text { Any new contracting of nonconcessional external borrowing } \\
\text { or guarantees by the Central Government and selected stateowned entities } \\
\text { (SOEs) subject to the related continuous quantitative AC will be for } \\
\text { transportation and electricity infrastructure investment, as described in } \\
\text { paragraph } 14 \text { of the MEFP dated May 24, } 2010\end{array}$ & Continuous & $\mathrm{Met}^{1 /}$ & $\begin{array}{l}\text { Ensure use of commercial borrowing for growth } \\
\text { enhancing investment so as to safeguard debt } \\
\text { sustainability. }\end{array}$ \\
\hline $\begin{array}{l}\text { The Minister of Planning and Development will issue a ministerial decree } \\
\text { requiring that a project selection protocol and a standard project } \\
\text { evaluation template be used by all Government entities in the analysis and } \\
\text { selection of public investment projects, as described in paragraph } 30 \text { of the } \\
\text { MEFP dated November } 8,2010 \text {. }\end{array}$ & $\begin{array}{l}\text { End-March } \\
\qquad 2011\end{array}$ & Met & $\begin{array}{l}\text { Improve the analysis and selection of public investment } \\
\text { projects, thereby supporting economic growth. }\end{array}$ \\
\hline $\begin{array}{l}\text { An action plan of priority measures will be adopted to simplify the tax } \\
\text { system, expand the tax base, and assess the merits and feasibility of } \\
\text { possibly reducing the corporate tax rates and of rationalizing incentives } \\
\text { under the tax benefits code, as described in paragraph } 31 \text { of the MEFP } \\
\text { dated November } 8,2010 \text {. }\end{array}$ & $\begin{array}{l}\text { End-March } \\
\qquad 2011\end{array}$ & Met & $\begin{array}{l}\text { Support envisaged further rise in revenue to-GDP ratio } \\
\text { and help contain the fiscal deficit. }\end{array}$ \\
\hline $\begin{array}{l}\text { The Government will issue two ministerial decrees to create a Debt } \\
\text { Management Committee (DMC) and a Project Coordination Committee } \\
\text { (PCC), as described in paragraph } 30 \text { of the MEFP dated November 8, } 2010 .\end{array}$ & $\begin{array}{l}\text { End-March } \\
\qquad 2011\end{array}$ & Met & $\begin{array}{l}\text { Enhance debt management and project selection, which } \\
\text { will reduce fiscal risks and support economic growth. }\end{array}$ \\
\hline $\begin{array}{l}\text { Building on the enhancements to e-FOLHA and e-CAF, a first quarterly } \\
\text { report will be issued on the civil servant population, wage payments by } \\
\text { sector, and the impact of promotion and recruitment decision at all levels, } \\
\text { as described in paragraph } 22 \text { of the MEFP dated November } 8,2010 \text {. }\end{array}$ & $\begin{array}{c}\text { End-July } \\
2011\end{array}$ & Met & $\begin{array}{l}\text { Strike the proper balance between modernizing the civil } \\
\text { service and keeping the wage bill in line with } \\
\text { macroeconomic stability. }\end{array}$ \\
\hline $\begin{array}{l}\text { The Government will submit to Parliament, a package of administrative } \\
\text { reforms to support the fight against corruption, including: (i) the revision of } \\
\text { anti-corruption law } 6 / 2004 \text {; (ii) the revisions of laws } 4 / 1990 \text { and } 7 / 1998 \text { on } \\
\text { the declaration of assets by government officials; (iii) the revision of law } \\
22 / 2007 \text { reforming the organic law of the public sector, in particular } \\
\text { regarding the competencies of the GCCC; and (iv) a new law to define and } \\
\text { regulate conflict of interest in the public service, as described in paragraph } \\
31 \text { of the MEFP dated May 20,2011. }\end{array}$ & $\begin{array}{l}\text { End-July } \\
2011\end{array}$ & $\begin{array}{l}\text { Met in } \\
\text { October }\end{array}$ & $\begin{array}{l}\text { Ensure adequate governance standards in promoting the } \\
\text { business environment and relations with development } \\
\text { partners. }\end{array}$ \\
\hline $\begin{array}{l}\text { The Bank of Mozambique will adopt the financial sector contingency plan's } \\
\text { modules related to (i) closing and liquidating banks, (ii) managing bidding } \\
\text { processes for private sector takeovers, (iii) bailouts, and (iv) emergency } \\
\text { liquidity assistance to promote rehabilitation of problem banks into viable } \\
\text { institutions, as described in paragraph } 34 \text { of the MEFP dated May 20, } 2011 .\end{array}$ & $\begin{array}{l}\text { End-November } \\
\qquad 2011\end{array}$ & $\begin{array}{l}\text { Partially } \\
\text { met }\end{array}$ & $\begin{array}{l}\text { Safeguard macroeconomic stability and the soundness } \\
\text { of the banking system. }\end{array}$ \\
\hline $\begin{array}{l}\text { The Minister of Planning and Development will approve an Integrated } \\
\text { Investment Program, in consultation with the Project Coordination } \\
\text { Committee, as described in paragraph } 25 \text { of the MEFP dated May 20, } 2011 .\end{array}$ & $\begin{array}{l}\text { End-March } \\
\qquad 2012\end{array}$ & Met & $\begin{array}{l}\text { Improve the selection of public investment projects, } \\
\text { thereby supporting economic growth. }\end{array}$ \\
\hline
\end{tabular}

1/ Discontinued at the 3rd Review. 


\section{APPENDIX I. LETTER OF INTENT}

May 10, 2012

Ms. Christine Lagarde

Managing Director

International Monetary Fund

Washington, D.C. 20431

U.S.A.

Dear Ms. Lagarde:

The Government of Mozambique requests the completion of the fourth review under the Policy Support Instrument (PSI). In support of this request, we are transmitting the attached Memorandum of Economic and Financial Policies (MEFP), which reviews implementation of our economic program under the PSI and sets out the Government's objectives and policies over the short and medium term.

The Government's economic program aims to maintain macroeconomic stability, promote more inclusive growth through public investment and the promotion of productive employment, and reduce poverty. The program aims to support the implementation of the new Poverty Reduction Strategy Paper (PARP) for 2011-14, which the Government formally adopted on May 3, 2011.

The Government is of the view that the policies outlined in the MEFP are adequate to achieve the objectives of the PSI-supported program. We stand ready to take any additional measures necessary to achieve our policy objectives. The Government will consult with the IMF-at its own initiative or whenever the Managing Director requests such a consultation-should revisions be contemplated regarding the policies contained in the attached MEFP. The Government will provide the IMF with 
such information as the IMF may request to be able to assess the progress made in implementing the economic and financial policies and achieving the objectives of the program.

Sincerely yours,

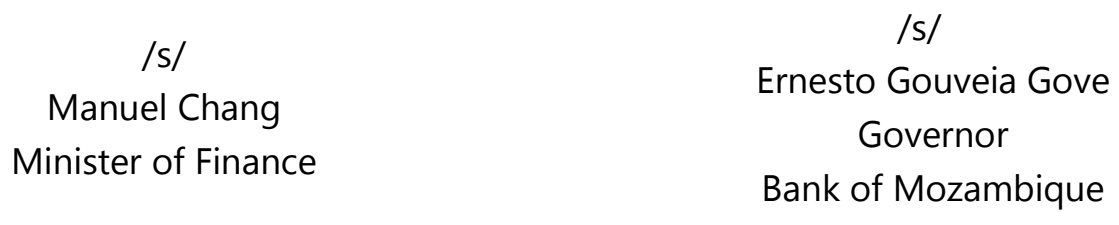

Attachments: Memorandum of Economic and Financial Policies

Technical Memorandum of Understanding 


\section{ATTACHMENT 1: MEMORANDUM OF ECONOMIC AND FINANCIAL POLICIES}

May 10, 2012

1. This updated MEFP (i) describes recent developments and performance under the Government's economic program under the three-year PSI to date and (ii) elaborates on economic and structural policy intentions for the remainder of 2012. It builds on the MEFPs underlying the initial PSI request dated May 28, 2010 and those for the first, second, and third reviews from November 8, 2010, May 20, 2011, and November 14, 2011, respectively.

\section{RECENT ECONOMIC DEVELOPMENTS AND PERFORMANCE UNDER THE PROGRAM}

\section{Mozambique's economy sustained a strong performance in 2011 despite a marked} deterioration in the global economic environment. Based on quarterly data, economic activity is estimated to have grown by 7.1 percent, in line with objectives. This result has been driven to a large extent by good harvests and a booming mining sector, reflecting the start of coal production. Twelve-month headline inflation decelerated sharply to 5.5 percent at end-2011 and 2.5 percent in February 2012, from its peak of 16.6 percent at end-2010. Inflation expectations have been effectively arrested, as core inflation (excluding food and fuel prices) declined to 5.9 percent at end2011 and further to 2.4 percent at end-February 2012. The determination of the authorities in tightening monetary and fiscal policies, coupled with the impact of favorable developments of international prices, a good harvest, and a stronger metical, contributed to the remarkable disinflation. In addition, preliminary external sector data indicate that exports and FDI have remained buoyant in spite of the more difficult external context, resulting in an external current account deficit of 13 percent of GDP and a better-than-expected level of international reserves equivalent to 4.8 months of imports.

\section{We largely complied with the objectives underlying our economic program:}

- All end-December 2011 quantitative assessment criteria (AC) were met comfortably, except for net credit to the government (NCG).

- The Bank of Mozambique (BM) succeeded in its disinflationary efforts by slowing reserve money (RM) growth from 291/4 percent in 2010 to $81 / 2$ in 2011, thus meeting the relevant end-December AC. It also observed, with ample margin, all quarterly targets on net international reserves (NIR) through end-December 2011. 
- The Government's fiscal policy stance in 2011 was supportive of the disinflation effort, especially in the first half of the year. However, both the end-September indicative target and the endDecember AC for NCG were marginally exceeded, in spite of buoyant revenue collections. This occurred as a Government guarantee for a public enterprise was called by a domestic bank. Excluding the outlays for the guarantee, we would have met both targets. The indicative target on priority spending for the year as a whole was met, notwithstanding having slightly underperformed at end-September.

- On nonconcessional borrowing (NCB), in addition to the loans for the rehabilitation of Maputo and Nacala airports, we have now signed a US $\$ 300$ million credit to build a ring road around Maputo, bringing our usage under the NCB program ceiling to US\$446 million. The ring road is intended to relieve urban congestion and facilitate private sector activity in the capital area.

- We made important progress in implementing the program's structural benchmarks. On March 26, the Minister of Planning and Development approved an Integrated Investment Program, which was developed in consultation with the Project Selection Committee (PCC). As to the endNovember 2011 benchmark on the financial sector contingency plan, we have adopted five of the envisaged six modules. However, recent Fund TA suggested several enhancements to make the plan more effective, which we will take up quickly.

\section{IMPLEMENTATION OF THE PARP}

\section{The Government has taken important steps to implement the new four-year PARP} adopted in May 2011. Consistent with the PARP's broad strategic vision, we have adopted a series of sectoral strategies and launched a variety of policy initiatives under the PARP's three main pillars that should help create the conditions for poverty reduction and broad-based inclusive growth. In addition, we succeeded in maintaining macroeconomic stability, as evidenced by this MEFP, and are taking important milestones in the governance area, as outlined below, which were envisaged under the PARP's supporting pillars. Among other things, our intentions under the PARP's main pillars are as follows:

- Enhancing production and productivity in agriculture. Framed by the Agricultural Reform Strategy (PEDSA) approved in May 2011, the Government is working toward doubling agricultural production by 2020 . This is to be achieved through the expansion of the agricultural frontier and an increase in productivity, with a focus on improving access to land, enhancing availability of high-quality seeds, fertilizers, and other inputs, and promoting farming techniques 
and market access. Among other things, the Government will expand the number of land leasing titles (DUATs) to 2,842 in 2012, from 2,701 in 2011. Moreover, it will aim to offer extension services to 10 percent of agricultural producers this year and to 15 percent in 2014, and set up silos in 5 provinces to improve market access. To help crowd in private investors and better prioritize public investment in rural areas, the Council of Ministers will adopt a new agricultural investment plan by end-June 2012, which will be disseminated in a road-show later this year.

- Creating employment. Several initiatives have been launched to create an environment that is more conducive to private sector activity. On February 2, the Government approved a decree simplifying the issuance of 84 types of licenses for 9 areas of activity. In early March, the Council of Ministers approved an action plan to accelerate business climate reforms for implementation in 2012, with clear lines of accountability. The plan includes a number of measures aimed to reduce by half the number of procedures to start a business. Other critical measures encompass the adoption of the bankruptcy code by Parliament, the completion of an inventory of business licenses, and the approval of a credit registry model. We are also well advanced in the preparation of the successor five-year Strategic Plan to Improve the Business Environment, which we expect to submit for Cabinet approval in the fourth quarter of 2012.

- Enhancing social and human development. The Government is on track to improve access to, and the quality of, social services and infrastructure. Among other things, to improve health care, we will raise the ratio of health workers to 100,000 inhabitants from 66 in 2012 to 69 in 2014. As to education, we aim to boost the net rate of schooling at age 6 in first grade from 73 to 79 percent during the period. As to social infrastructure, line ministries are stepping up their investment plans to improve access to water, basic sanitation, electricity, dwelling, and urban transport services. Finally, supported by the ILO, UNICEF, the Bank, and the Fund, we are in the process of enhancing our social protection systems (see below).

\section{MACROECONOMIC POLICIES}

\section{A. Economic Objectives}

\section{We expect Mozambique's medium-term outlook to remain positive, although the} current global economic environment poses risks in the short run. Our objective is to sustain high economic growth of $7 \frac{1}{2}$ to 8 percent over the next five years. Nonetheless, while we also retain this objective for 2012, the more difficult external environment could temporarily lower economic 
growth by up to one percentage point. This could in particular be the result of a moderation in external demand and capital inflows which may affect domestic economic activity. Nonetheless, the external current account deficit is set to improve slightly this year and hover at around 12 percent of GDP during the next five years, while the reserves coverage of imports should exceed 5 months. Barring any unforeseen external price shocks, we are confident to achieve our inflation objective of 5 to 6 percent during the period.

\section{B. Macroeconomic Policy Mix}

\section{We remain committed to a prudent macroeconomic policy mix over the medium term.}

Fiscal and monetary policies will be geared toward safeguarding macroeconomic stability and debt sustainability while fostering the Government's investment and social development goals so as to sustain high and inclusive economic growth. The fiscal policy stance will accommodate a temporary surge in public investment over the next few years to close the infrastructure gap and support the PARP's social and human development objectives, including the expansion of social protection. While this stance will boost the overall fiscal deficit to 5 to $61 / 2$ percent of GDP during the period, the strong revenue effort and restraint in domestic current spending will limit the domestic primary deficit to 2 to $2 \frac{1}{2} 2$ percent of GDP. The continued strong support of development partners and NCB within the program ceiling will ensure that recourse to domestic bank financing will not exceed 1 percent of GDP, thus providing ample room for private sector credit expansion. Monetary policy will be sufficiently accommodating to support this objective while primarily aiming to preserve low inflation. The envisaged rise in money demand will keep RM expansion slightly faster than nominal GDP growth.

\section{Our policy stance for $\mathbf{2 0 1 2}$ will cushion the impact of a temporary global slowdown} while safeguarding the recent disinflation gains. Given the ample policy buffers as a result of our strong track record of prudent macroeconomic policies, the Government intends to embark on a more countercyclical policy stance for 2012. The BM's policy response will entail a continued cautious easing of monetary policy consistent with the annual inflation objective.

\section{The pace of our temporary boost in infrastructure investment, partially financed by} NCB, will be compatible with maintaining macroeconomic stability and debt sustainability. In light of this, we will implement our investment priorities within a raised NCB program ceiling of US $\$ 1.5$ billion. Our own updated debt sustainability analysis suggests that Mozambique will continue to enjoy a low risk of debt distress as long as the envisaged NCB-financed investment remains focused in transportation and energy infrastructure, is time-limited, and geared toward projects with a proven economic return. We will base our investment decisions on our recently completed Integrated Investment Plan and thorough feasibility studies and cost-benefit-analyses, 
generally also including an assessment of lower-cost alternatives. We consider this stepping up of public investment financed by nonconcessional borrowing to be temporary, since over the long run we expect to begin reaping higher revenues from the natural resource sector that are expected to create the necessary fiscal space to accommodate our investment and other priorities.

\section{Monetary and Exchange Rate Policies}

\section{The monetary policy stance will become more accommodating in 2012 but is not} expected to jeopardize the annual inflation objective of 5 to 6 percent. Such a policy stance will support the provision of private sector credit at a critical time. We project RM growth to accelerate to about 20 percent, exceeding nominal GDP growth by one-fourth, thus contributing to the deepening of the financial system. Following the strong signaling effect of our recent easing of key policy rates, the BM will continue to be guided in its future policy decisions by its inflation objective.

\section{The authorities will continue to give utmost priority to enhancing the current} framework for monetary policy operations. This entails the following specific measures:

- Monitoring government accounts. The implementation of the MoU signed on March 25, 2011 on the exchange of information between the BM and the Ministry of Finance (MoF) is proceeding well and the objectives from the last MEFP have been achieved. Both institutions will continue to improve the timely exchange of information.

Developing the domestic repo market to help improve money market management and the liquidity of government securities. The BM is on track to implement the measures envisaged in the November 14, 2011 MEFP for implementation by end-July 2012 to improve the functioning of the interbank and repo markets. Following Fund TA advice, it will also turn the FPD credit facility into a repo operation during 2012 so as to shift market preference from uncollateralized to collateralized operations. We are also in the process of assessing the impact of possible measures to equalize the tax treatment between BT and OT and between collateralized and uncollateralized interbank market operations. As indicated in the MEFP dated November 14, 2011, we will reflect any such equalization measures in an amendment to the Corporate Income Tax Law to be submitted to Parliament by end-September 2012 to take effect in 2013 (structural benchmark).

\section{The BM will continue to prepare for the adoption of an inflation-targeting (IT)} framework in the medium term. Measurable progress in monetary policy formulation and implementation has been achieved, with support from Fund TA, to lay the foundations for an IT framework. The BM will continue to improve data collection and analysis, forecasting modeling, 
forward-looking communication, and managing short-term liquidity forecasting. In addition, we are on track to publish by end-May 2012 our first quarterly monetary policy report to enhance communication and transparently and efficiently explain the BM's policy intentions (structural benchmark).

\section{The authorities renew their commitment to maintaining a flexible exchange rate}

regime. To this effect, the BM will allow the exchange rate to adjust freely to evolving patterns of trade and financial flows and closely monitor developments in the real effective exchange rate vis-àvis a broad basket of currencies, with a view to ensuring external competitiveness and a comfortable level of international reserves.

\section{The BM will ensure that Mozambique remains committed to its obligations under} Article VIII, sections 2, 3, and 4 of the Fund's Articles of Agreement. The BM will continue to monitor the implementation of the new foreign exchange regulations, its implementation norms, as well as the functioning of the foreign exchange market, with a view to avoiding practices that could turn out to be inconsistent with Article VIII principles. We will keep IMF staff informed of any developments that could potentially run against those principles.

\section{Fiscal Policy}

14. The countercyclical fiscal policy stance for $\mathbf{2 0 1 2}$ will entail a slight increase in the fiscal deficit. Although the Revenue Authority will strive to collect the tax revenue targets under the 2012 budget law, revenue collections may fall short of these objectives in light of the possibly less favorable economic environment. However, the Government intends to maintain its spending program to avoid aggravating the economic and social effects of the global downturn and to pursue the country's transformation agenda under the PARP. As a result, the domestic primary deficit could rise by 0.2 percentage points relative to earlier projections, to $2 \frac{1 / 4}{4}$ of GDP, in 2012. This higher deficit would be fully financed by additional World Bank financing, thus avoiding crowding out the private sector from domestic bank financing.

\section{A further projected drop in revenues would, however, trigger application of the} provisions which limits budget execution to cautionary ceilings consistent with the Organic Budget Law (SISTAFE 2002). This will be based on the decree on the provisions regarding budget execution (Delegação de Competência sobre a Excecução do Orçamento; Decree No. 1/2011) adopted by the Council of Ministers on February 2, 2012 and the ministerial circular (Circular Ministerial No 4/2012) issued by the Minister of Finance on December 30, 2011. 


\section{The implementation of our salary policy will remain gradual but supportive of the} recruitments needed for the PARP priority sectors. In line with the review of the salary policy implementation strategy completed at end-March 2012, we will decelerate the decompression of the salary scale; decelerate the pace of recruitment in non-priority areas and institutions while emphasizing the accumulation of human capital; and strengthen the execution of the wage bill through a rapid expansion of the electronic salary payment system (e-FOLHA) in the public sector. These measures will lead to a slight decrease of the wage bill to around $81 / 2$ per cent of GDP over the medium term. As of end-February, the Government has rolled-out the electronic salary payment system e-FOLHA to 152 provincial directorates and is confident to complete the rollout to all 172 directorates by end-June 2012 (structural benchmark), and to the entire central level by end-2012. It will also continue to produce quarterly wage bill reports for use by the Ministries of Finance and Civil Service to monitor and control salary expenditures.

17. Spending for priority sectors under the PARP will be increased. The Government aims to rise such spending from MT 58 billion in 2011 to MT 73 billion in 2012, or from 50 to 53 percent of projected total expenditures (excluding net lending). Such spending has been redefined to incorporate outlays related to social action, labor and employment, consistent with the Government's enhanced focus on those areas under the new PARP. The Government will complete the full mapping of priority spending with the PARP's three main and two supporting pillars by endJuly 2012.

\section{We will support implementation of the PARP by expanding social protection while} moving toward eliminating the costly fuel subsidy. In this endeavors, we are drawing on the expertise of our development partners.

- Enhancing social protection. The Government is working to support the implementation of the revamped social protection programs approved by the Council of Ministers in September 2011 and financed under the 2012 budget. The Ministry for Women and Social Action (MMAS) is strengthening its operational systems for the effective delivery of cash transfers and the launch of public works programs. We expect the first public works pilots to be launched in 2 cities and 4 provinces this year. MMAS continues to revise its targeting and beneficiary identification mechanisms, payment procedures, and monitoring and evaluation systems. The Government is also working with development partners on the establishment of a common fund for social protection to guarantee the complementary funding needed to finance the requisite investments in institutional capacity and the medium-term expansion of the beneficiaries envisaged under the programs. MMAS and MoF will regularly assess the costing and affordability of increasing the amount of the existing transfers that will generate improvements 
in the living conditions of beneficiaries. Government allocations to social protection will rise from 0.4 at present to 0.8 percent of GDP over the next few years when the Government's envisaged target of 815,000 beneficiaries under both the cash transfer and public works programs could possibly be achieved. Under the best circumstances, the fiscal space for social protection could further rise to 1.4 percent of GDP early in the next decade.

- Phasing out the fuel subsidy. The Government remains committed to fully eliminating the costly fuel subsidy at the earliest possible moment. While cost recovery was effectively achieved early in 2012, the subsequent global oil price increases have again caused a need for compensation for fuel importers. In the short run, the Government will aim to contain such need for compensation by exploring options for cross-subsidization of various petroleum products through greater segmentation of the market and relatively more frequent and smaller price adjustments than in the past. With a more medium-term perspective, it is reviewing avenues to improve the price-setting formula that has temporarily been suspended. We will complete paying compensation to fuel importers for incurred 2011 losses by end-May 2012, consistent with the agreement reached with them.

\section{STRUCTURAL REFORMS}

\section{A. Public Financial Management and Reporting}

\section{We are committed to improving the reporting, monitoring, and control of budget}

execution. The following measures are considered key:

- Expanding budget coverage and system integration. The rollout of the e-SISTAFE budget execution system will be completed by 2015 covering 98 percent of all spending, as per the September 2011 rollout plan. On this basis, we aim to increase the e-SISTAFE coverage to 47 percent this year. We have completed an action plan for 2012-14, to be adopted by end-May 2012 covering among other things the development of IT hardware and software, and technical skills among staff.

- Enhancing the quality in PFM processes. Notwithstanding our substantial efforts in developing and implementing SISTAFE, there is scope to improve the real-time recording of the appropriation, liquidation, and payment phases consistent with the effective delivery of goods and services. Based on Fund TA and recent studies by the General Financial Inspectorate, we are implementing measures to reinforce control and audit functions and step up training at the 
provincial level. We expect to have full compliance with real-time recording of the expenditure chain by 2013.

- Migrating to IPSAS-compatible reporting. We will gradually reform the State Account format and content according to international public accounting standards (IPSAS). We will prepare the 2011-13 State Account reports under the old and new format, in tandem with efforts to continue to improve data quality and compiling mechanisms. To this end, the Minister of Finance will approve a time-bound action plan for IPSAS-compatible reporting by end-October 2012 (structural benchmark). This time-bound action plan will clearly define the procedures required to elaborate the State Account of 2014 in the new IPSAS-compatible format. Success of this reform hinges crucially on strengthening capacity at the Accounting Directorate.

- Expanding fiscal decentralization. We will further decentralize fiscal responsibilities with due regard to local capacity, proper internal controls, and available financing. To this effect, we are gradually rolling out a district development monitoring system (SMdDD) aimed at assessing districts' administrative performance. We have assessed 33 districts in 2011, and our goal is to assess 80 districts in 2012 and all 128 districts in 2013. We will make these assessments public to local district councils and communities.

\section{B. Debt Management and Investment Planning}

20. The Government has reached important milestones in modernizing debt management and investment planning. Our reform efforts will continue in both areas.

- Following up on Integrated Investment Plan. We completed the Integrated Investment Plan laying out our strategic vision for investment priorities, describing selection procedures, and providing our current assessment of a list of top priority projects. We consider this plan a rolling document which we will update in light of further analysis, focusing in particular on projects' expected economic and social returns and lower-cost alternatives.

- Completing DSA and MTDS. We completed our second annual debt sustainability analysis (DSA) in early-May 2012, which should allow us to finalize and submit for approval by the Council of Ministers our enhanced medium-term debt strategy (MTDS) by end-June 2012, some three months behind our initial schedule.

- Benefitting from advisory committees. The Debt Management Committee (DMC) and Project Coordination Committee (PCC) have met quarterly and issued regular reports to the respective supervising minister. The PCC also supervised the effective application of the new project 
selection template and the drafting of the Integrated Investment Plan. The PCC will benefit from the adoption of the project preparation manual, which the Government expects to adopt by end-May 2012, with support from the World Bank. In turn, the DMC provided guidance in the drafting of the MTDS and our second DSA.

- Strengthening MoF debt unit. Preparation of the DSA benefitted from a complete overhaul of our debt database. To preserve data quality, improve management of the country's debt service, ensure a high-quality analysis of financing proposals, and support the DMC, the unit will complete an operating procedures manual by end-June 2012 for approval by the Minister of Finance. The manual will assign clear roles and responsibilities to the unit's staff, and establish norms for data entry and validation and other processes. The debt unit has started producing quarterly debt reports since the last quarter of 2011 and will continue this practice going forward. Starting in June 2012, these reports will include up-to-date information on the evolution of public and publicly-guaranteed debt, the contracting of loans, cost and risk indicators, and a link to the scenarios underlying the new MTDS. These reports will be made available to support DMC decision making and monitoring of the debt management strategy.

- Completing the first Annual Domestic Borrowing Plan for 2013. Building on the MTDS, we will draw up and publish a first annual domestic borrowing plan for 2013 upon approval of that year's budget law but no later than end-January. The plan will improve internal planning and help communications with the market, thereby reducing potential issuance cost in the long term. It will include quarterly financing and repayment projections and be consistent with the projected net annual financing needs and cash flow throughout the year (structural benchmark).

\section{Tax Administration}

\section{We will sustain our strong revenue mobilization effort while aiming to make the tax} system more business-friendly. This will mainly be achieved through refinements in tax policy and the adoption of modern tax administration practices.

- Implementing the single taxpayer database and registration number. Based on the structural benchmark to make the database functional by end-June 2012, we expect that by end-2012, some 80 percent of corporate taxpayers and 20 percent of individual taxpayers will be registered in the database throughout the country. 
- Enhancing tax payment efficiency. We will revise our tax legislation to accommodate the implementation of the electronic tax system (e-tax). This will be done for the VAT by end-2012 and for the large corporate and personal income taxes by end -June 2013. We will take this opportunity to address the current tax refund inefficiencies related to the personal income tax and VAT and revise, and possibly eliminate, stamp tax obligations on administrative acts, based on a recent study.

- Fostering tax payments through banks. Following the recent adoption of the Action Plan for Tax Payments through Banks, the Government will introduce the payment through banks for custom duties, VAT, and simplified tax for small tax payers (ISPC) by end-2013 and all income taxes by July 2014.With technical support from development partners, we will strive to accelerate the implementation schedule.

- Reinforcing the large taxpayers unit (LTD-DCAT). Modern management procedures in the LTD-DCAT will continue to be reinforced through the large taxpayer's liaison agents and a dedicated data collection information system. The latter will be fully operational by end-June 2012. The unit is expected to build expertise on natural resources and strategic sectors over time to enable targeted audits and help improve revenue forecasting. Collections by the LTD-DCAT are projected to rise from 56 percent in 2011 to 58 percent in 2012, 63 percent in 2013, and 70 percent in 2014.

- Completing rollout of single-window trade facilitation project (JUE). Following its launch last year to streamline custom declaration procedures at the Port of Maputo, the JUE will be rolled out to the country's seven main entry points by end-2012 and collect 70 percent of all customs revenues. We expect that this share will rise to 90 percent end-2013.

\section{Improving Natural Resource Management}

\section{Great strides have been made to bring Mozambique very close to full EITI}

membership. The second reconciliation country report was completed in mid-March and presented to stakeholders at a March 30 seminar. The report fully addressed the guidance from the EITI Secretariat and represents a major leap toward the adoption of a best-practice framework to improve the transparency of the natural resource sector.

\section{The Government is enhancing its technical and institutional capacity to manage} natural resources, with support from partners. Under forthcoming Fund TA under the Topical Trust Fund for Managing Natural Resources Wealth (TTF-MNRW), we will review and implement reforms to our mining and hydrocarbon fiscal regime to ensure that an appropriate share of benefits 
is captured for Mozambique under newly launched projects while remaining an attractive investment destination. In particular, we will look into methods for the appropriate valuation of mineral assets for fiscal purposes and revisit the taxation of capital gains. Our intention is to submit the revised legislation to Parliament expeditiously, possibly as early as end 2012.

\section{Other challenges in natural resource management will be addressed in due course. We}

will enhance mining and hydrocarbon revenue forecasting, assess the feasibility of creating a stabilization fund to support the country's long-term development strategy, and improve the statistics on natural resources. While respecting all existing Government commitments, we are exploring opportunities for mutually beneficial renegotiations of terms under existing natural resources sector projects where changes in circumstances may have led to a misalignment.

\section{E. Good Governance Framework}

\section{The Government will ensure the proper implementation of the anti-corruption} legislation upon its approval by Parliament. This will enhance the investment climate and instill confidence of local and international stakeholders. To this effect, and with technical support from our partners, the Government will produce in due course a time-bound and costed action plan, including all the institutional reforms required to fully implement the anti-corruption legislation package. Looking at anti-corruption practices more broadly, the Government will update the 200610 Governance Anti-Corruption Strategy by end-July 2012, based on all available studies and relevant documents, such as the recently completed Second National Study on Governance and Corruption Analysis, the 2009-14 Integrated Justice Strategy, and the report on the implementation of the African Peer Review Mechanism (MARP) in Mozambique. It will be implemented, and also disseminated to and discussed with key stakeholders. Our PARP matrix will be revised accordingly to incorporate key measures under the action plan to implement the anti-corruption legislative package and the broader Governance Anti-Corruption Strategy.

\section{FINANCIAL SECTOR POLICIES}

\section{A. Financial Sector Surveillance}

\section{The $B M$ is intensifying its financial sector surveillance in response to the global}

financial turmoil. Among other things, the central bank is focusing on:

- Strengthening supervision. With Fund TA guidance, the BM is re-assessing the banking sector's vulnerabilities to tensions in the Euro area and considering options to lessen risks for crossborder contagion. Further steps to enhance risk-based banking supervision will also be taken through the migration to Basel II, which should be concluded in 2014. 
- Completing financial sector contingency plan. The authorities aim to adjust the draft plan by incorporating recent Fund TA recommendations, especially with respect to the modules on emergency liquidity assistance and bank resolution. We expect to have the full plan operational by end-September 2012.

- Making Deposit Insurance Fund (DIF) operational. We aim to make the DIF, created in 2010, an integral component of our framework to safeguard financial sector stability. To this end, we will, in coordination with the MoF, assess options to secure its funding and decide on deposit coverage expeditiously.

\section{B. Fighting Money Laundering and Terrorism Financing}

27. The AML/CFT framework is expected to be operational shortly. The MoF has established, chaired, and appointed the members of the high-level AML/CFT National Task Force in December 2011. The AML/CFT law was approved by the Council of Ministers and formally submitted to Parliament on April 19, 2012. The law is needed to make the AML/CFT framework fully effective and allow other regulators to adopt implementing guidelines. The AML/CFT strategic plan, prepared by the financial intelligence unit GIFIM, was approved by the Council of Ministers on April 17, 2012. The strategic plan will aim to foster national, regional, and international cooperation, strengthen the AML/CFT institutional framework, and promote capacity building and outreach efforts.

\section{Financial Sector Development Strategy}

\section{We are close to officially launching Mozambique's Financial Sector Development}

Strategy (FSDS) for 2011-20. The strategy includes well-defined measures to enhance financial inclusion, competition, consumer protection, and financial literacy, thereby supporting Mozambique's long-term economic growth. The three strategic and operational bodies (Steering Committee, Technical Advisory Committee, and Implementation Unit) were set up in November 2011 and have become fully operational. The FSDS approval by the Council of Ministers was delayed to May 2012 to incorporate recommendations by the Steering Committee. We intend to launch the FSDS' dissemination in June through a workshop organized by the World Bank and are working with development partners to fund the implementation of the FSDS.

\section{Payment System}

29. The BM will further enhance financial stability and promote market development through payment system reforms. Following the rollout of the real time gross settlement system in national currency (MTR) in 2010, it has worked on improving the monitoring of risks in the electronic clearing system (CEL) for checks, electronic fund transfers, financial settlement of securities. Based on 
ongoing TA, the BM will adopt a payment systems oversight policy strategy and procedures manual by end-August 2012. It will also complete the self-assessment of the MTR and CEL against the Core Principles for Systemically Important Payment Systems by end-August 2012. Going forward, a particular focus will be on launching a retail payments network shared by the BM and commercial banks. Following the establishment of the retail payments company SIMO in June 2011, a pilot phase is expected to be completed during 2012 after which the platform is expected to handle all retail transactions and support other interbank services, such as electronic funds transfers and mobile banking.

\section{PROGRAM MONITORING}

30. The proposed modifications of quantitative ACs and indicative targets for end-June 2012, the proposed indicative targets for end-September 2012, and the proposed ACs and indicative targets for end-December 2012 are shown in Table 1. Table 2 provides a list of the proposed structural benchmarks. The fifth PSI review is expected to be completed by end-2012 and the sixth and last PSI review by June 13, 2013. 
Table 1. Mozambique: Quantitative Assessment Criteria and Indicative Targets ${ }^{1}$ (Millions of meticais, unless otherwise specified)

\begin{tabular}{|c|c|c|c|c|c|c|c|c|c|c|c|c|c|}
\hline & & & & & & & & & & & & & \\
\hline & \multicolumn{7}{|c|}{2011} & \multirow{4}{*}{ Status } & \multicolumn{5}{|c|}{2012} \\
\hline & \multirow{2}{*}{\multicolumn{3}{|c|}{$\begin{array}{l}\text { End-Sept } \\
\text { Indicative Target }\end{array}$}} & \multirow{3}{*}{ Status } & \multirow{2}{*}{\multicolumn{3}{|c|}{ End-Dec }} & & \multirow{2}{*}{$\begin{array}{l}\text { End-March } \\
\text { Indicative } \\
\text { Tarqet }\end{array}$} & \multirow{2}{*}{\multicolumn{2}{|c|}{$\begin{array}{l}\text { End-June } \\
\text { Assessment } \\
\text { Criteria }\end{array}$}} & \multirow{3}{*}{$\begin{array}{c}\begin{array}{c}\text { End-Sept } \\
\text { Indicative } \\
\text { Target }\end{array} \\
\text { Proposed } \\
\end{array}$} & \multirow{3}{*}{$\begin{array}{c}\text { End-Dec } \\
\text { Assessment } \\
\text { Criteria } \\
\text { Proposed }\end{array}$} \\
\hline & & & & & & & & & & & & & \\
\hline & Prog. & Adj. & Act. & & Prog. & Adj. & Act. & & Prog. & Prog. & Proposed & & \\
\hline \multicolumn{14}{|l|}{ Assessment Criteria for end-June/December } \\
\hline Net credit to the government (cumulative ceiling) & -362 & -362 & -74 & NM & 2,896 & 3,066 & 3,360 & NM & 2,795 & $-2,645$ & $-2,645$ & -745 & 2,500 \\
\hline Stock of reserve money (ceiling) & 33,708 & 33,400 & 31,436 & M & 36,053 & 36,053 & 33,275 & M & 33,800 & 36,734 & 36,923 & 37,031 & 39,985 \\
\hline Stock of net international reserves of the BM (floor, US\$ millions) & 1,905 & 1,905 & 2,130 & M & 2,030 & 2,038 & 2,238 & M & 2,091 & 2,154 & 2,207 & 2,323 & 2,438 \\
\hline New nonconcessional external debt contracted or guaranteed by the central & & & & & & & & & & & & & \\
\hline $\begin{array}{l}\text { government or the BM or selected state-owned enterprises with maturity of one year } \\
\text { or more (cumulative ceiling over the duration of the program, US\$ millions) }\end{array}$ & 900 & 900 & 146 & M & 900 & 900 & 146 & M & 900 & 900 & 1,500 & 1,500 & 1,500 \\
\hline Stock of short-term external public debt outstanding (ceiling) & 0 & 0 & 0 & M & 0 & 0 & 0 & M & 0 & 0 & 0 & 0 & 0 \\
\hline External payments arrears (ceiling, US\$ millions) & 0 & 0 & 0 & M & 0 & 0 & 0 & M & 0 & 0 & 0 & 0 & 0 \\
\hline \multicolumn{14}{|l|}{ Indicative targets } \\
\hline Government revenue (cumulative floor) & 56,382 & 56,382 & 60,490 & M & 76,792 & 76,792 & 81,119 & M & 20,272 & 45,556 & 45,256 & 69,687 & 94,568 \\
\hline Priority spending (cumulative floor) & 40,839 & 40,839 & 40,589 & NM & 58,000 & 58,000 & 58,242 & M & 10,500 & 24,500 & 24,500 & 35,934 & 72,563 \\
\hline
\end{tabular}

1 For definition and adjustors, see the Program Monitoring Section of the Memorandum of Economic and Financial Policies and the Technical Memorandum of Understanding. 
Table 2. Mozambique: Structural Benchmarks Under the PSI

\begin{tabular}{|c|c|}
\hline Structural Benchmarks & $\begin{array}{l}\text { Expected Date of } \\
\text { Implementation }\end{array}$ \\
\hline $\begin{array}{l}\text { The Bank of Mozambique will publish its first quarterly monetary policy report, as } \\
\text { described in paragraph } 11 \text { of the MEFP dated November 14, } 2011 \text {. }\end{array}$ & End-May 2012 \\
\hline $\begin{array}{l}\text { The Government will complete the rollout of the e-Folha electronic salary } \\
\text { payment system to all provincial directorates, as described in paragraph } 19 \text { of the } \\
\text { MEFP dated November } 14,2011 \text {. }\end{array}$ & End-June 2012 \\
\hline $\begin{array}{l}\text { The Ministry of Finance will make fully functional a new single taxpayer database } \\
\text { and identification number, as described in paragraph } 23 \text { of the MEFP dated } \\
\text { November } 14,2011 \text {. }\end{array}$ & End-June 2012 \\
\hline $\begin{array}{l}\text { The Government will submit to Parliament an amendment to the Corporate } \\
\text { Income Tax Law reflecting measures derived from an impact study on the } \\
\text { equalization of the tax treatment between Treasury bills and Government bonds } \\
\text { and between collateralized and uncollateralized interbank market operations, to } \\
\text { take effect in } 2013 \text {, as described in paragraph } 10 \text { of the MEFP dated November } \\
14,2011 .\end{array}$ & End-September 2012 \\
\hline $\begin{array}{l}\text { The Minister of Finance will approve a time-bound action plan for IPSAS- } \\
\text { compatible reporting, as described in paragraph } 19 \text { of this MEFP. }\end{array}$ & End-October 2012 \\
\hline $\begin{array}{l}\text { The Government will adopt an Annual Borrowing Plan for 2013, as described in } \\
\text { paragraph } 20 \text { of this MEFP. }\end{array}$ & End-January 2013 \\
\hline
\end{tabular}




\section{ATTACHMENT 2: TECHNICAL MEMORANDUM OF UNDERSTANDING}

May 10, 2012

1. This Technical Memorandum of Understanding (TMU) defines the quantitative assessment criteria, indicative targets, and structural benchmarks on the basis of which the implementation of the Fund-supported program under the Policy Support Instrument (PSI) will be monitored. In addition, the TMU establishes the terms and timeframe for transmitting the data that will enable Fund staff to monitor program implementation.

\section{DEFINITIONS}

\section{A. Net credit to the central government}

2. Net credit to the central government (NCG) by the banking system is defined as the difference between the outstanding amount of bank credits to the central government and the central government's deposits with the banking system, excluding deposits in project accounts with the banking system, recapitalization bonds issued to the Bank of Mozambique (BM), and proceeds from the signing fee for mineral resource exploration. Credits comprise bank loans, advances to the central government and holdings of central government securities and promissory notes. NCG will be calculated based on data from balance sheets of the monetary authority and commercial banks as per the monetary survey. The limits on the change in NCG by the banking system will be cumulative from end-December of the previous year.

3. The central government encompasses all institutions whose revenue and expenditure are included in the state budget (Orçamento do Estado): central government ministries, agencies without financial autonomy, and the administration of 11 provinces. Although local governments (43 municipalities or autarquias) are not included in the definition because they are independent, part of their revenue is registered in the state budget as transfers to local governments.

4. For program purposes, net disbursements on the nonconcessional Portuguese credit line are excluded from the assessment criterion of NCG since the corresponding expenditure is not covered under the definition of central government specified in paragraph 3. 


\section{B. Government revenue and financing}

5. Revenue is defined to include all receipts of the General Directorate of Tax (Direç̧ão Geral dos Impostos, DGI), the General Directorate of Customs (Direcção Geral das Alfândegas, DGA), and nontax revenue, including certain own-generated revenues of districts and some line ministries, as defined in the budget. Net receipts from privatization received by the National Directorate of State Assets (Direcção Nacional do Património do Estado) and unrealized profits transferred by the central bank to the treasury will not be considered as revenue (above the line) and will be accounted for as other domestic financing (below the line).

6. For the purpose of program monitoring, revenue is considered as collected at the time when it is received by the relevant government collecting agencies, in cash or checks, or through transfers into the respective bank account.

\section{Priority social spending}

7. Priority social spending is based on the PARPA program categories expanded to incorporate all areas under the new PARP. Accordingly, it will include total spending in the following sectors: (i) education; (ii) health; (iii) HIV/AIDS; (iv) infrastructure development; (v) agriculture; (vi) rural development; a (vii) governance and judicial system, and (viii) social action, labor and employment.

\section{Reserve money}

8. For the purposes of program monitoring reserve money is defined as the sum of currency issued by the BM and commercial banks' holdings at the BM. The target is defined in terms of the average of the daily end-of-day stocks in the month of the test date. The reserve money stock will be monitored and reported by the BM.

\section{E. Net international reserves}

9. Net international reserves (NIR) of the BM are defined as reserve assets minus reserve liabilities. The BM's reserve assets include (a) monetary gold; (b) holdings of SDRs; (c) reserve position at the IMF; (d) holdings of foreign exchange; and (e) claims on nonresidents, such as deposits abroad (excluding the central government's savings accounts related to mineral resource extraction concessions). Reserve assets exclude assets pledged or otherwise encumbered, including but not limited to assets used as collateral or guarantee for a third-party external liability (assets not 
readily available). The BM's reserve liabilities include: (a) all short-term foreign exchange liabilities to nonresidents with original maturity of up to and including one year; and (b) all liabilities to the IMF.

\section{F. New nonconcessional external debt contracted or guaranteed by the central government, the BM, and selected state-owned enterprises, with maturity of more than one year}

10. The ceiling on nonconcessional external debt applies to external debt contracted or guaranteed by the central government, the BM, the Road Fund, the water authorities (FIPAG), and the electricity company (EDM). It also applies to debt contracted by these three state-owned enterprises from domestic banks or from other state-owned enterprises that is contractually interrelated to external nonconcessional loans.

11. The ceiling applies to external debt with original maturity of one year or more and with a grant element below 35 percent. The grant element is calculated using currency-specific discount rates based on the Organization for Economic Cooperation and Development (OECD) commercial interest reference rates in accordance with the annual Budget Law. The term 'debt' will have the meaning set forth in Point 9 of the Guidelines on Performance Criteria with Respect to External Debt in Fund Arrangements adopted on August 3, 1979, as amended August 31, 2009, effective December 1, 2009. The concept of external debt is defined on the basis of the residency of the creditor. The ceiling also applies to commitments contracted or guaranteed for which value has not been received. This assessment criterion is defined cumulatively from the beginning of the program and will be assessed on a continuous basis.

\section{G. Stock of short-term external public debt outstanding}

12. The central government will not contract or guarantee external debt with original maturity of less than one year. This assessment criterion applies not only to debt as defined in Point 9 of the Guidelines on Performance Criteria with Respect to External Debt in Fund Arrangements adopted on August 3, 1979, as amended August 31, 2009, effective December 1, 2009, but also to commitments contracted or guaranteed for which value has not been received. Excluded from this assessment criterion are short-term, import-related trade credits. This assessment criterion will be assessed on a continuous basis. 


\section{H. External payments arrears}

13. The government undertakes not to incur payments arrears on external debt owed or guaranteed by the central government, with the exception of external payments arrears arising from government debt that is being renegotiated with creditors. This assessment criterion will be assessed on a continuous basis.

\section{Foreign program assistance}

14. Foreign program assistance is defined as grants and loans received by the Ministry of Finance through BM accounts excluding those related to projects (Table 1).

\section{J. Actual external debt service payments}

15. Actual external debt service payments are defined as cash payments on external debt service obligations of the government and central bank, including obligations to Paris Club and other bilateral creditors rescheduled under enhanced HIPC Initiative completion point terms, multilateral creditors, and private creditors, but excluding obligations to the IMF (Table 1).

\section{ADJUSTERS}

\section{A. Net international reserves}

16. The quantitative targets (floors) for net international reserves (NIR) will be adjusted:

- downward by the shortfall in external program aid less debt service payments (up to US\$100 million), compared to the program baseline (Table 1);

- downward/upward for any revision made to the end-year figures corresponding to the previous year; and

- downward to accommodate higher external outlays because of natural disasters, up to US\$20 million.

\section{B. Net credit to central government}

17. The quantitative targets (ceilings) for net credit to the central government (NCG) will be adjusted: 
- upward by the shortfall in the MT value of external program aid receipts less debt service payments (up to the MT equivalent of US $\$ 100$ million at exchange rates prevailing at the respective test dates), compared to the program baseline (Table 1);

- downward by privatization proceeds in excess of those envisaged in the program, unless these proceeds are deposited in the government's savings accounts abroad;

- downward (upward) for any increase (decrease) in domestic financing from the nonfinancial private sector; and

- upward to accommodate the higher locally-financed outlays because of natural disasters, up to the MT equivalent of US $\$ 20$ million at exchange rates prevailing at the respective test dates.

\section{Reserve money}

18. The quantitative target (ceiling) for reserve money will be adjusted upward by the excess of the stock of currency in circulation above the level envisaged in the program. For programming purposes, both the stocks of reserve money and currency in circulation are defined in terms of the average of the daily end-of-day stocks in the month of the test date. The target will be adjusted up to MT 750 million for end-December 2011 and up to MT 250 million for end-March, end-June, endSeptember and end-December 2012 (Table 1).

19. The ceiling on reserve money for every test date will be adjusted downward/upward to reflect decreases/increases in the legal reserve requirement on the liabilities in commercial banks. The adjuster will be calculated as the change in the reserve requirement coefficient multiplied by the amount of commercial banks' liabilities subject to reserve requirement, considered at the end of the period of constitution of the required reserves prior to the change in regulation.

\section{DATA AND OTHER REPORTING}

20. The Government will provide Fund staff with:

- monthly and quarterly data needed to monitor program implementation in relation to the program's quantitative targets and broader economic developments;

- weekly updates of the daily data set out in Table 1;

- weekly data set out in Table 4 of the TMU dated May 26, 2005; 
- monthly updates of the foreign exchange cash flow of the BM;

- monthly data on government revenues (in detail according to the fiscal table) with a lag not exceeding one month;

- monthly information on the balance of government savings accounts abroad;

- monthly data on domestic arrears;

- monthly data on external arrears;

- monthly budget execution reports (that will also be published) with a time lag not exceeding 45 days;

- the "mapa fiscal" with a time lag not exceeding 60 days;

- monthly monetary survey data with a time lag not exceeding 30 days;

- monthly data on gross international reserves, with the composition by original currencies and converted to US dollars at the actual exchange rates; and

- quarterly balance-of-payments data with a time lag not exceeding 65 days;

- monthly disbursements on the nonconcessional Portuguese credit line with a time lag not exceeding 30 days.

21. The monetary survey made available by the BM will clearly identify donor-financed project deposits (with a breakdown between foreign and domestic currency) included in net credit to the government in both the central bank's and commercial banks' balance sheets.

22. The government will provide Fund staff with documentation concerning external loan agreements once these have been signed and become effective. 
TMU Table 1. Mozambique: Net Foreign Assistance, 2011-12

\begin{tabular}{|c|c|c|c|c|c|c|c|c|}
\hline & \multicolumn{4}{|c|}{2011} & \multicolumn{4}{|c|}{2012} \\
\hline & \multicolumn{2}{|l|}{ Q3 } & \multicolumn{2}{|c|}{ Q4 } & \multirow{2}{*}{$\begin{array}{c}\text { Q1 } \\
\text { Prog. }\end{array}$} & \multirow{2}{*}{$\begin{array}{c}\text { Q2 } \\
\text { Proj. }\end{array}$} & \multirow{2}{*}{$\begin{array}{c}\text { Q3 } \\
\text { Proj. }\end{array}$} & \multirow{2}{*}{$\begin{array}{c}\text { Q4 } \\
\text { Proj. }\end{array}$} \\
\hline & Prog. & Act. & Prog. & Act. & & & & \\
\hline Net foreign program assistance (US\$ mn) & 104 & 51 & 36 & 37 & 70 & 223 & 3 & 63 \\
\hline Gross foreign program assistance & 119 & 77 & 51 & 52 & 94 & 251 & 30 & 90 \\
\hline Program grants & 119 & 77 & 19 & 21 & 94 & 141 & 30 & 59 \\
\hline Program loans & 0 & 0 & 32 & 31 & 0 & 110 & 0 & 32 \\
\hline External debt service & 15 & 26 & 15 & 14 & 24 & 27 & 27 & 27 \\
\hline Net foreign program assistance (MT mn) & 3,170 & 1,400 & 925 & 966 & 2,032 & 6,699 & 81 & 1,981 \\
\hline Gross foreign program assistance & 3,693 & 2,141 & 1,433 & 1,334 & 2,753 & 7,548 & 930 & 2,855 \\
\hline Program grants & 3,693 & 2,141 & 547 & 501 & 2,753 & 4,235 & 930 & 1,857 \\
\hline Program loans & 0 & 0 & 886 & 833 & 0 & 3,313 & 0 & 998 \\
\hline External debt service & 523 & 741 & 508 & 368 & 722 & 849 & 848 & 874 \\
\hline Stock of outstanding currency (MTm) & 22,512 & 20,065 & 23,650 & 21,899 & 21,230 & 23,440 & 24,222 & 27,593 \\
\hline Stock of outstanding currency (MTm), Prog. Def & 21,901 & 19,802 & 23,600 & 21,168 & 21,499 & 22,995 & 23,905 & 26,672 \\
\hline
\end{tabular}

Source: Mozambican authorities and IMF staff estimates. 


\section{APPENDIX II. RESERVE ADEQUACY ASSESSMENT ${ }^{1}$}

\section{Mozambique's reserve accumulation has been broadly in line with the growth of the}

economy. Reserves grew more than threefold over the past decade. In nominal terms, gross international reserves reached USD 2.4 billion at end-2011 (Figure 1.a), which is estimated at 5.2 months of current-year imports. Mozambique's steady reserves accumulation mirrors the accelerated built-up across LICs since 2002. The significant boost to Mozambique's reserves to 5.4 months of imports in 2009 resulted from the SDR allocation and the use of Fund resources under the Exogenous Shock Facility (ESF). Reserve growth has been broadly in line with the growth of the economy while it was outstripped by financial sector deepening (Figure 1.b). ${ }^{2}$

\section{Mozambique has accumulated reserves at a somewhat higher pace than a majority of}

Sub-Saharan African countries. Based on the traditional metrics of the reserve coverage ratio in terms of months of imports and broad money, Mozambique's reserves have been broadly comparable to the regional average (Figure 1.c, 1.e). But the country's percentile ranking across SubSaharan Africa, while on a declining trend, shows that Mozambique has constantly been amongst countries holding more reserves (Figure 1.d, 1.f). This has been helped by substantial aid flows: On average, gross aid inflows to the central government accounted for more than 12 percent of GDP over the period under review (2003-11).

\section{Mozambique's level of reserves} is adequate when measured against the recently proposed cost-benefit analysis on reserve adequacy for LICs. ${ }^{3}$ As opposed to traditional metrics which measure the country's exposure to one type of risk, the new metric balances the

Variables

\begin{tabular}{lr}
\hline Government balance, \% of GDP (2003-11) & -3.9 \\
CPIA (2007-10) & 3.7 \\
External demand growth, percent (2003-11) & -0.3 \\
TOT growth, percent (2003-11) & -2.3 \\
Change in FDI to GDP, percentage point (2003-11) & -2.7 \\
Change in aid to GDP, percentage point (2003-11) & -2.3 \\
\hline
\end{tabular}
adequacy of the level of reserves pertaining to the probability and the severity of various shock events with an estimate of the cost of holding reserves. The shock values (changes in the terms of trade, external demand, FDI and aid) are set at the bottom tenth percentile of the distribution to

\footnotetext{
${ }^{1}$ Prepared by Patrick Gitton.

${ }^{2}$ The traditional short-term debt metric is not reported because of shortcomings on short-term external debt data.

${ }^{3}$ See Dabla-Norris et al. (2011) http://www.imf.org/external/pubs/cat/longres.aspx?sk=25316.0, and IMF (2011)

"Assessing Reserve Adequacy" http://www.imf.org/external/np/pp/eng/2011/021411b.pdf
} 
capture the size of an extreme shock. The standard approach shows that a level above 5 months of imports is adequate for Mozambique whose cost of holding reserves is comparatively low given the concessional nature of most of its external debt. The soundness of the country's fundamentals reflected in fiscal performance and the quality of public management and institutions (CPIA) have a mitigating impact on the probability and the depth of economic shocks.

4. Increasing the level of reserves would be consistent with upcoming structural changes in the Mozambican economy. The appreciable amount of international reserves held by the Bank of Mozambique has helped shield the country against external shocks, in particular the 2008 commodity price crisis. The likelihood of declining aid flows from traditional donors over the medium term, as well as the transformation of the economy into a major commodity exporter, will increase the country's needs to protect against price volatility and exogenous shocks. This is reflected in staff's medium-term reserves projection of 5.9 months of current-year imports by 2017. 
Figure 1. Mozambique: International Reserves

a. Gross International Reserves, Billions USD

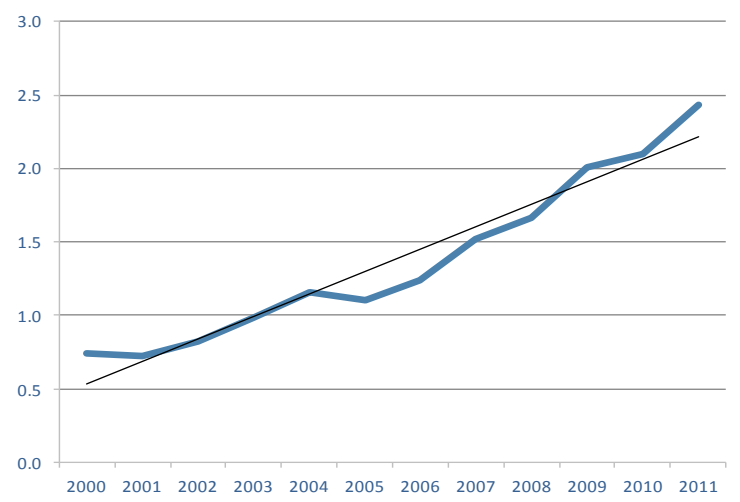

c. Reserves/months of current year imports

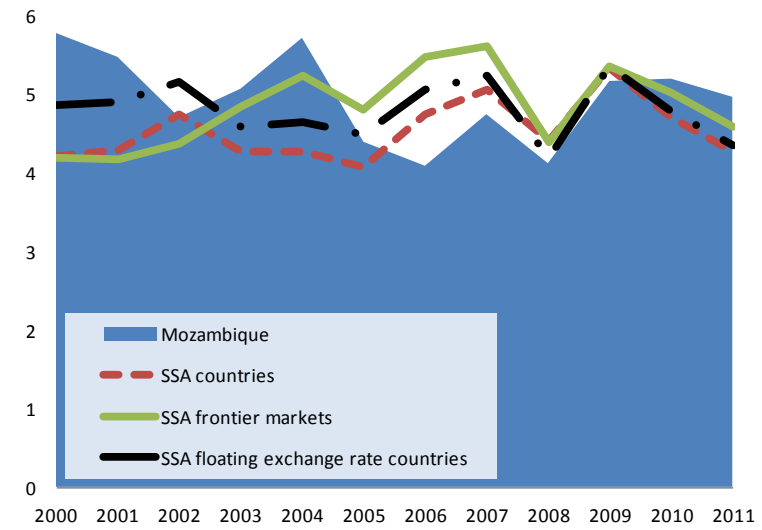

e. Reserves/M2

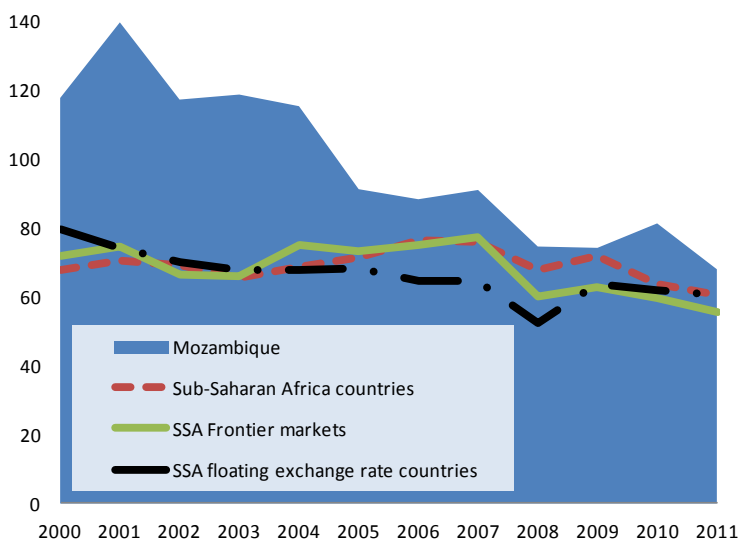

b. Reserve adequacy metrics, percent of GDP

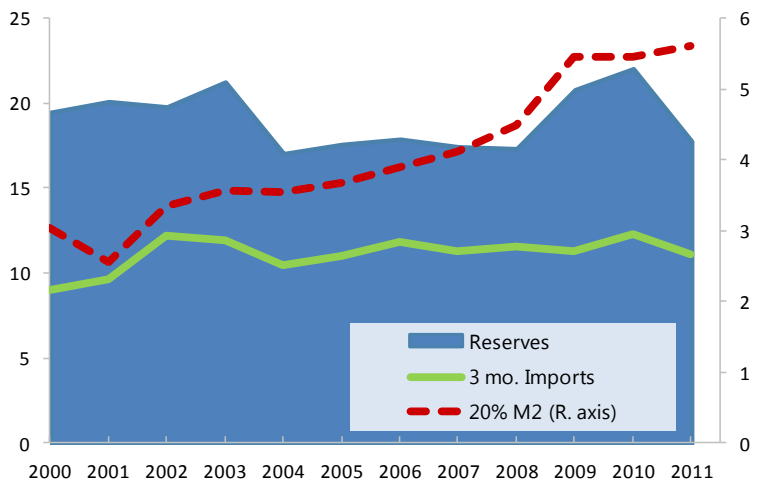

d. Reserves/months of current year imports (Mozambique percentile rank in SSA)

100

80

60

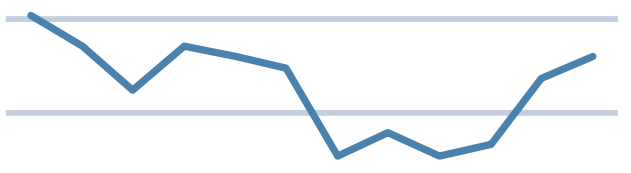

20

200020012002200320042005200620072008200920102011

f. Reserves/M2 (Mozambique percentile rank in SSA)

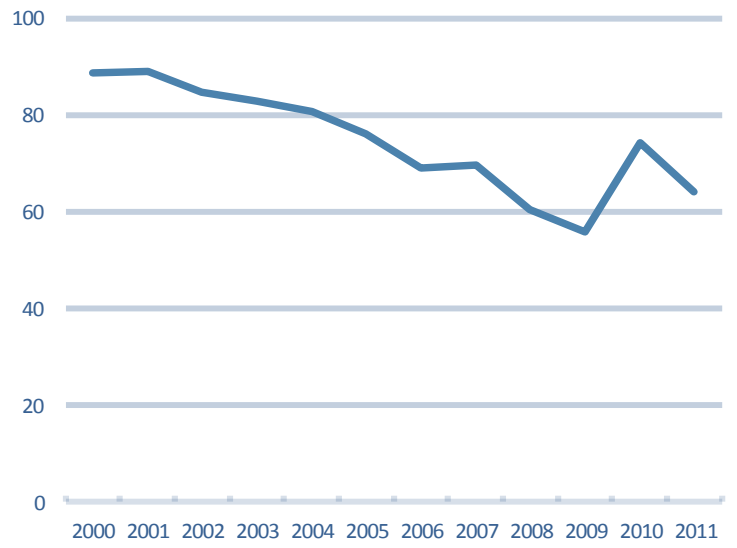

Sources: WEO, World Economic Outlook, and AFR-REO, Regional Economic Outlook.

Aggregate data reflect the simple average across countries.

Frontier economies: Angola, Ghana, Kenya, Mauritius, Mozambique, Nigeria, Senegal, Tanzania, Uganda, Zambia. 


\title{
INTERNATIONAL MONETARY FUND
}

\section{REPUBLIC OF MOZAMBIQUE}

\author{
FOURTH REVIEW UNDER THE POLICY SUPPORT
}

May 11, 2012 INSTRUMENT AND REQUEST FOR MODIFICATION OF ASSESSMENT CRITERIA-DEBT SUSTAINABILITY ANALYSIS

\author{
Approved By \\ Saul Lizondo and Vivek \\ Arora (IMF) and Marcelo \\ Guigale and Jeffrey \\ Lewis (World Bank)
}

Prepared by the staffs of the International Monetary Fund and the International Development Association

This debt sustainability analysis (DSA) updates the joint IMF/IDA DSA from May 24, 2011 reflecting the most recent macroeconomic developments. ${ }^{9}$ Consistent with the previous DSA, its main results indicate that Mozambique remains at a low risk of debt distress as the government plans to temporarily increase public investment partially financed by external borrowing on nonconcessional terms over the next few years. However, further improvements in debt management and project selection capacity will be key to use nonconcessional resources productively. ${ }^{10}$ As public debt is largely external, the evolution of public debt indicators mirrors that of external debt.

\footnotetext{
${ }^{9}$ In line with the 2010 Staff Guidance Note, a full joint LIC DSAs is expected to be prepared once every three years for PRGT-eligible IDA-only countries. In between, short annual updates are expected to be produced unless macroeconomic conditions since the last full DSA have significantly changed. See Staff Guidance Note on the Application of the Joint Fund-Bank Debt Sustainability Framework for Low-Income Countries (www.imf.org) and IDA/SECM2010-0029.

${ }^{10}$ The DSA presented in this document is based on the standard low-income countries (LIC) DSA framework. See Debt Sustainability in Low-Income Countries: Further Considerations on an Operational Framework, Policy Implications (www.imf.org) and IDA/SECM2004/0629. Under the Country Policy and Institutional Assessment (CPIA), Mozambique is rated as a medium performer, with an average rating of 3.71 during 2008-10, and the DSA uses the indicative threshold for countries in this category.
} 


\section{UNDERLYING DSA ASSUMPTIONS}

5. This DSA is consistent with the macroeconomic framework outlined in the Staff Report for the Fourth Review under the Policy Support Instrument. Compared to the previous DSA, ${ }^{11}$ this update includes:

\section{a. A revised medium-term}

macroeconomic framework. It takes into account a temporary, albeit small, dent in economic growth in 2012 as a result of the global downturn, but a slightly improved outlook over the medium to long term as a result of booming investment and production in the mining and hydrocarbon sectors. The analysis also assumes, like in the past, that Mozambique will continue its prudent macroeconomic policy mix, with a broadly stable domestic primary fiscal deficit, helped by a sustained revenue administration effort. The external current account deficit is projected to gradually improve, as buoyant growth of exports from megaprojects in the natural resource sector is likely to outpace import growth. Stronger-than-previouslyprojected FDI inflows, reflecting the booming mining sector and new discoveries in the hydrocarbon sector, should continue over the medium term before tapering off in the long run.

\footnotetext{
${ }^{11}$ See IMF Country Report No. 11/149.
}

b. A revised public investment profile.

This reflects slower-than-previouslyprojected disbursements of the nonconcessional external borrowing (NCB) contracted at the time of the previous DSA, revised projections reflecting new NCB contracted recently, and the proposed increase in the NCB ceiling to US $\$ 1.5$ billion under the IMF-supported program to finance projects considered critical under the authorities' public investment plan. The loans contracted under the NCB ceiling are assumed to be disbursed throughout the next four years. ${ }^{12}$ Disbursements under the Portuguese credit line have also been updated reflecting realized disbursements.

\section{c. Revised grant financing projections,} reflecting a lower grant component in external financing in line with the increase of the NCB ceiling.

\footnotetext{
${ }^{12}$ To date, authorities have signed three nonconcessional loans: (i) in December 2010, a loan from China amounting US\$66 million to modernize Maputo airport; (ii) in April 2011, a loan from Brazil for the construction of Nacala airport of US $\$ 80$ million, and (iii) in February 2012, a US $\$ 300$ million loan from China for the construction of a ring road around Maputo.
} 


\begin{tabular}{|c|c|c|c|c|c|}
\hline \multicolumn{6}{|c|}{$\begin{array}{c}\text { Text Table 1. Evolution of selected macroeconomic } \\
\text { indicators between DSA updates }\end{array}$} \\
\hline \multicolumn{6}{|c|}{ Evolution of selected macroeconomic indicators } \\
\hline & Actual & \multirow{2}{*}{$\begin{array}{r}\text { Preelim. } \\
2011\end{array}$} & \multicolumn{2}{|c|}{ Projection } & \multirow[b]{2}{*}{2014} \\
\hline & 2010 & & 2012 & 2013 & \\
\hline \multicolumn{6}{|c|}{ Real GDP growth (\%) } \\
\hline Previous DSA & 6.6 & 7.2 & 7.5 & 7.9 & 7.8 \\
\hline Current DSA & 6.8 & 7.1 & 6.7 & 7.2 & 7.8 \\
\hline \multicolumn{6}{|c|}{ Nominal GDP (US\$ mill) } \\
\hline Previous DSA & 9.8 & 12.1 & 13.5 & 15.1 & 16.5 \\
\hline Current DSA & 9.5 & 12.8 & 14.2 & 15.5 & 17.3 \\
\hline \multicolumn{6}{|c|}{ Primary fiscal deficit (\% of GDP) } \\
\hline Previous DSA & 2.9 & 5.5 & 5.8 & 5.5 & 4.8 \\
\hline Current DSA & 3.1 & 4.0 & 5.2 & 5.2 & 5.2 \\
\hline \multicolumn{6}{|c|}{ Current account deficit (\% of GDP) } \\
\hline Previous DSA & 10.2 & 11.4 & 10.9 & 10.9 & 10.7 \\
\hline Current DSA & 11.7 & 13.1 & 12.7 & 12.4 & 11.9 \\
\hline \multicolumn{6}{|l|}{ FDI (\% of GDP) } \\
\hline Previous DSA & 8.1 & 7.2 & 7.1 & 6.5 & 7.0 \\
\hline Current DSA & 8.3 & 16.4 & 11.2 & 10.2 & 10.1 \\
\hline \multicolumn{6}{|c|}{ Use of NCB ceiling ( $\%$ of GDP) } \\
\hline Previous DSA & - & 2.1 & 2.4 & 2.2 & 1.8 \\
\hline Current DSA & - & 1.5 & 2.5 & 3.2 & 3.4 \\
\hline \multicolumn{6}{|c|}{ Disbursements under PTL (\% of GDP) $1 /$} \\
\hline Previous DSA & 1.3 & 1.9 & 1.4 & 1.1 & 0.8 \\
\hline Current DSA & 1.4 & 1.8 & 0.9 & 0.9 & 0.5 \\
\hline \multicolumn{6}{|c|}{ Grant financing (\% of GDP) $2 /$} \\
\hline Previous DSA & $\ldots$ & 9.3 & 9.0 & 8.9 & 8.8 \\
\hline Current DSA & $\ldots$ & 9.2 & 8.7 & 8.1 & 8.0 \\
\hline \multicolumn{6}{|c|}{$\begin{array}{l}\text { 1/ Portuguese credit line. } \\
\text { 2/ Grant-equivalent financing includes grants provided directly to the government and } \\
\text { through new borrowing (difference between the face value and the PV of new } \\
\text { disbursements). }\end{array}$} \\
\hline
\end{tabular}




\section{EXTERNAL DEBT SUSTAINABILITY ANALYSIS}

6. Under the baseline scenario, all debt indicators remain well below their respective thresholds, including in the longer term, but external debt remains vulnerable to exchange rate volatility (Table 1 and Figure 1). The debt indicators are projected to increase over the next few years, as the authorities temporarily step up their borrowing to address the country's infrastructure gap. Stress tests reveal that Mozambique's external debt is particularly vulnerable to a one-time depreciation of the exchange rate and a negative exports shock. ${ }^{13}$

\section{The debt sustainability indicators} also seem sensitive to a recurrence of past macroeconomic performance. However, as indicated in previous DSAs, basing the analysis on a recurrence of developments of the last ten years does not take into account the significant structural changes in the Mozambican economy in the post-civil war period and the considerable improvements in the macroeconomic environment under successive Fund-supported programs, all of which have lessened the likelihood of a repeat of the past economic performance. Among other things, there has been a shift in the structure of the economy, particularly in the

\footnotetext{
${ }^{13}$ In line with the last two DSAs, the standard export shock has been tailored to capture Mozambique's true vulnerability from an export shock. This is represented by the historical volatility of prices of aluminum, which accounts for roughly half of Mozambique's export proceeds.
}

last five years, as large private capital inflows in the natural resources sector supported a surge in exports and a sharp fall in the current account deficit. The significant surge in capital inflows in recent years is expected to continue over the medium term, reflecting the current expansion of investment and output in the mining sector and additional gas-related discoveries which will trigger further investment and output growth over the long term. ${ }^{14}$ Therefore, staff does not consider the historical scenario a reliable indicator of future debt distress in Mozambique as it underestimates future growth prospects and current account improvements linked to the positive impact from investment in the natural resource sector.

\section{In a scenario under which non-} concessional resources are not used productively, external debt indicators would deteriorate, but remain well below their respective thresholds. The customized high investment-low growth scenario assumes that foreign financing and related spending remain unchanged relative to the baseline in nominal terms. However, it assumes a much lower impact on GDP growth over the projection period from the public investment projects compared to the baseline. Although

\footnotetext{
${ }^{14}$ For a more detailed explanation of the impact of different megaprojects, see IMF CR No. 11/350, Annex II.
} 
debt burden indicators are projected to remain below the indicative thresholds, the substantial increase relative to the baseline underscores the importance of selecting projects with high economic returns, particularly when using non concessional financing.

\section{PUBLIC SECTOR DEBT SUSTAINABILITY}

\section{The evolution of public debt} indicators (including domestic debt) mirrors that of the external indicators because of the predominance of external debt (Table $3 a$ and Figure 2). The medium-term increase in public debt reflects the temporary surge in public investment financed by external borrowing on non-concessional terms. However, over the longer term, the public debt stock projections also assume a marginal

\section{CONCLUSIONS}

\section{Mozambique's external debt} dynamics continue to show a low risk of debt distress. Its external and public debt levels are expected to remain well below the respective indicative thresholds for debt distress, as the government plans to temporarily increase public investment financed by external borrowing on nonconcessional terms. Nonetheless, debt vulnerability will increase, as debt ratios under increase in domestic debt from about 2.2 percent of GDP at end-2010 to 5.7 per-cent of GDP in $2032{ }^{7}$

\section{Over time, since the surge in public} investment is expected to taper off, the primary deficit should moderate as well. However, as scenario A2 shows, continued high primary deficits could pose a risk to public debt sustainability.

stress tests and under the high-investment/low growth scenario approach the relevant thresholds. This emphasizes the need for the authorities to continue to pursue prudent macroeconomic policies and structural reforms to boost their debt management and project selection capacity, building on the initial reform steps in those areas under their IMFsupported program and those implemented with World Bank assistance.

\footnotetext{
${ }^{7}$ This is not expected to affect the availability of credit to the private sector.
} 
Figure 1. Mozambique: Indicators of Public and Publicly Guaranteed External Debt Under Alternative Scenarios, 2012-2032 ${ }^{1}$
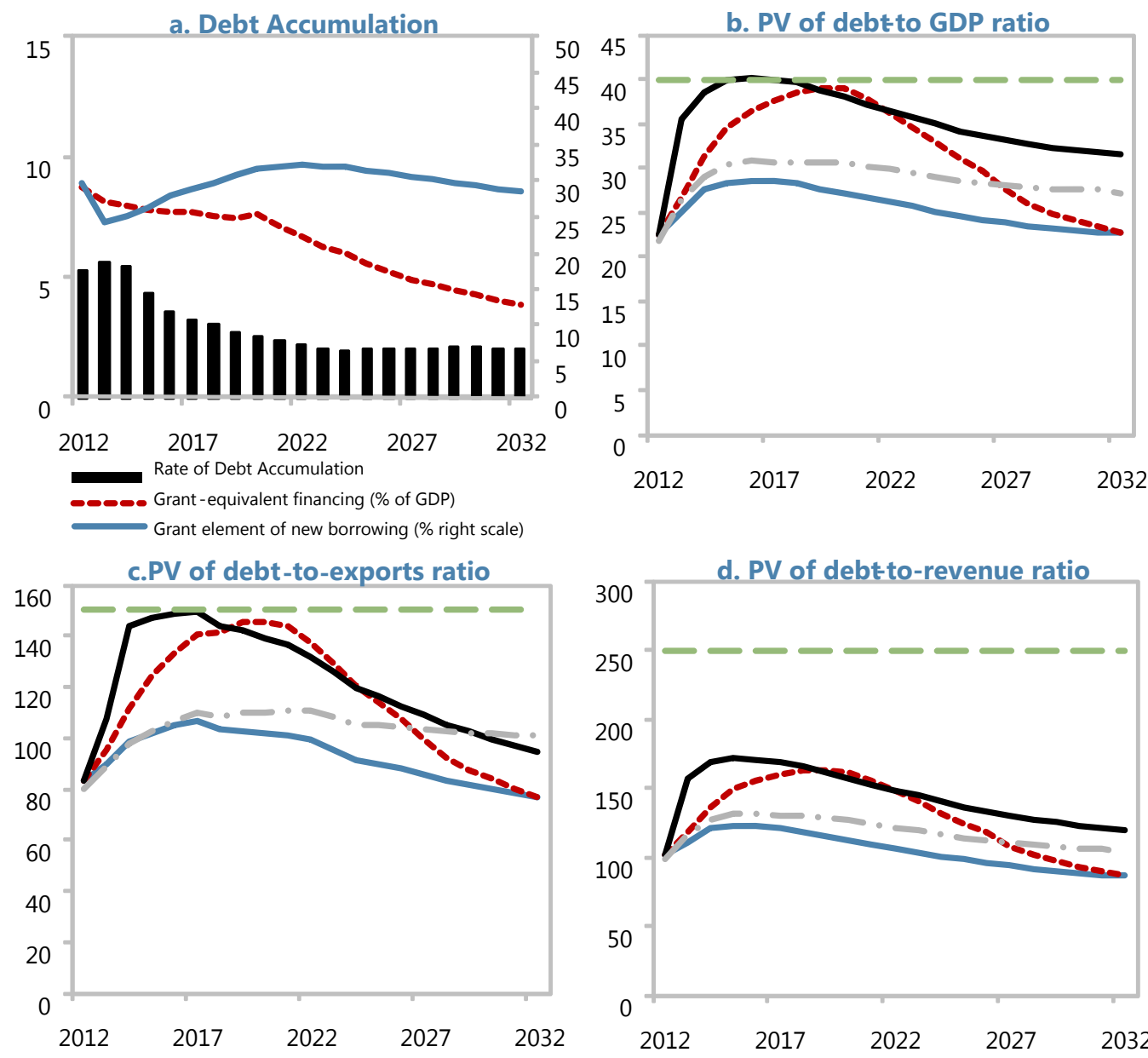

b. PV of debtto GDP ratio

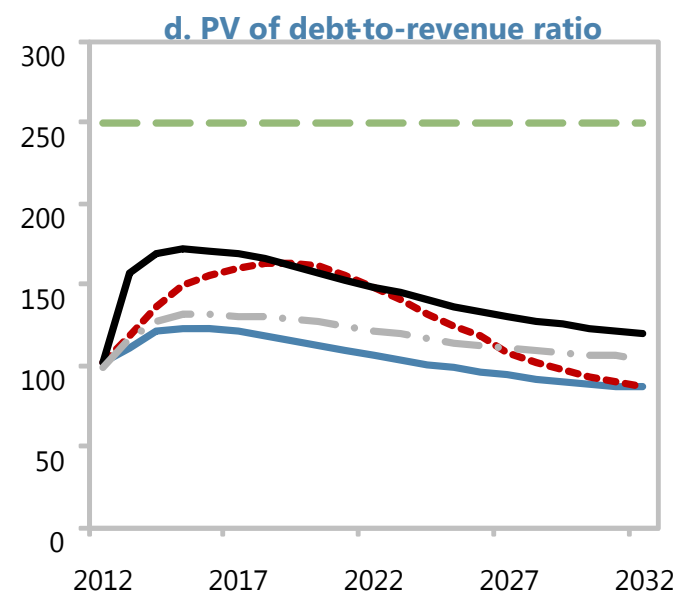

e. Debt serviceto-exports ratio

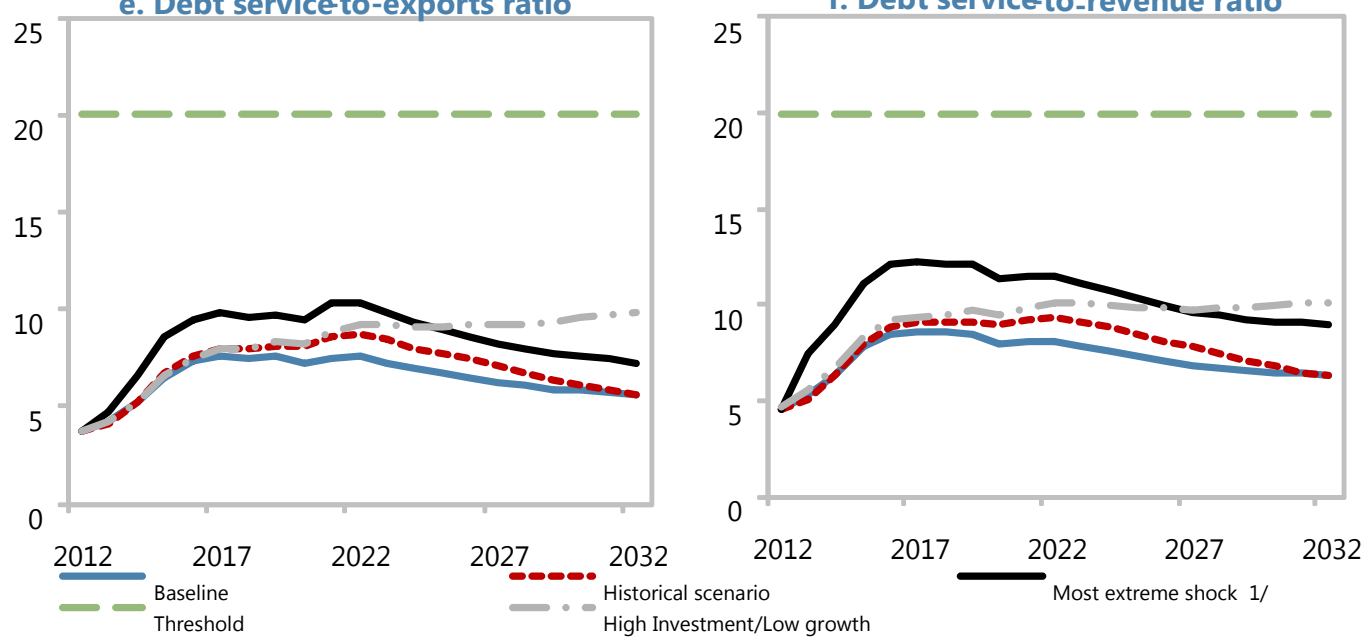

Sources: Country authorities; and staff estimates and projections.

$1 /$ The most extreme stress test is the test that yields the highest ratio in 2022. In figure b. it corresponds to a One-time depreciation shock; in c. to a Exports shock; in d. to a One-time depreciation shock; in e. to a Exports shock and in figure f. to a One-time depreciation shock. 
Table 1. External Debt Sustainability Framework, Baseline Scenario, 2009-2032 ${ }^{1}$ (In percent of GDP, unless otherwise indicated)

\begin{tabular}{|c|c|c|c|c|c|c|c|c|c|c|c|c|c|c|c|}
\hline & \multicolumn{3}{|c|}{ Actual } & \multicolumn{2}{|c|}{ Historical $^{6 /}$ Standard ${ }^{6 /}$} & \multicolumn{6}{|c|}{ Projections } & \multirow[b]{2}{*}{$2012-2017$} & \multirow{2}{*}{\multicolumn{3}{|c|}{$2018-2032$}} \\
\hline & \multirow[b]{2}{*}{2009} & \multirow[b]{2}{*}{2010} & \multirow[b]{2}{*}{2011} & \multirow[t]{2}{*}{ Average } & \multirow[t]{2}{*}{ Deviation } & \multirow[b]{2}{*}{2012} & \multirow[b]{2}{*}{2013} & \multirow[b]{2}{*}{2014} & \multirow[b]{2}{*}{2015} & \multirow[b]{2}{*}{2016} & & & & & \\
\hline & & & & & & & & & & & 2017 & Average & 2022 & 2032 & Average \\
\hline External debt $1 /$ & 64.3 & 64.3 & 50.5 & & & 53.1 & 52.7 & 52.8 & 51.7 & 50.9 & 50.3 & & 48.6 & 53.7 & \\
\hline o/w public and publicly guaranteed (PPG) & 38.4 & 39.0 & 31.5 & & & 38.3 & 40.6 & 43.1 & 43.4 & 43.5 & 43.1 & & 39.6 & 33.4 & \\
\hline Change in external debt & -2.0 & 0.0 & -13.9 & & & 2.7 & -0.4 & 0.1 & -1.2 & -0.8 & -0.6 & & -0.2 & 0.9 & \\
\hline Identified net debt-creating flows & 3.2 & 6.7 & -19.9 & & & -1.6 & -1.2 & -1.8 & -2.2 & -1.5 & -1.2 & & 1.3 & 2.0 & \\
\hline Non-interest current account deficit & 10.7 & 10.1 & 11.7 & 10.7 & 3.4 & 11.2 & 10.9 & 10.4 & 9.9 & 9.6 & 9.2 & & 9.3 & 9.4 & 9.1 \\
\hline Deficit in balance of goods and services & 17.4 & 17.8 & 17.3 & & & 15.8 & 14.9 & 13.9 & 12.9 & 12.3 & 11.7 & & 10.3 & 10.6 & \\
\hline Exports & 27.7 & 31.4 & 27.0 & & & 27.0 & 28.0 & 28.0 & 27.8 & 27.3 & 26.8 & & 26.4 & 29.7 & \\
\hline Imports & 45.1 & 49.2 & 44.3 & & & 42.7 & 42.9 & 42.0 & 40.7 & 39.6 & 38.5 & & 36.7 & 40.2 & \\
\hline Net current transfers (negative $=$ inflow) & -7.7 & -6.9 & -5.8 & -7.5 & 2.9 & -4.8 & -4.5 & -4.3 & -4.3 & -4.2 & -4.0 & & -2.8 & -1.9 & -2.6 \\
\hline o/w official & -6.8 & -6.4 & -5.7 & & & -4.6 & -4.4 & -4.2 & -4.2 & -4.1 & -3.9 & & -2.7 & -1.8 & \\
\hline Other current account flows (negative $=$ net inflow) & 1.0 & -0.7 & 0.2 & & & 0.2 & 0.6 & 0.8 & 1.3 & 1.5 & 1.5 & & 1.8 & 0.7 & \\
\hline Net FDI (negative $=$ inflow) & -8.9 & -8.3 & -16.4 & -6.8 & 4.2 & -11.2 & -10.2 & -10.1 & -9.7 & -8.8 & -8.1 & & -6.0 & -5.2 & -5.7 \\
\hline Endogenous debt dynamics 2/ & 1.4 & 4.9 & -15.2 & & & -1.7 & -2.0 & -2.2 & -2.3 & -2.3 & -2.2 & & -2.0 & -2.1 & \\
\hline Contribution from nominal interest rate & 1.6 & 1.6 & 1.4 & & & 1.4 & 1.5 & 1.5 & 1.4 & 1.3 & 1.3 & & 1.3 & 1.5 & \\
\hline Contribution from real GDP growth & -4.2 & -4.6 & -3.4 & & & -3.1 & -3.5 & -3.7 & -3.7 & -3.6 & -3.5 & & -3.3 & -3.6 & \\
\hline Residual (3-4) 3/ & -5.2 & -6.7 & 6.0 & & & 4.3 & 0.8 & 1.9 & 1.0 & 0.7 & 0.6 & & -1.5 & -1.1 & \\
\hline o/w exceptional financing & -4.8 & -4.3 & -5.4 & & & 0.0 & 0.0 & 0.0 & 0.0 & 0.0 & 0.0 & & 0.0 & 0.0 & \\
\hline PV of external debt 4/ & $\ldots$ & $\ldots$ & 36.5 & & & 37.3 & 37.2 & 37.3 & 36.6 & 36.0 & 35.7 & & 35.3 & 42.9 & \\
\hline In percent of exports & $\ldots$ & $\ldots$ & 135.0 & & & 138.5 & 132.8 & 133.3 & 131.5 & 132.0 & 133.1 & & 133.6 & 144.8 & \\
\hline PV of PPG external debt & $\ldots$ & $\ldots$ & 17.5 & & & 22.5 & 25.1 & 27.6 & 28.3 & 28.6 & 28.5 & & 26.2 & 22.7 & \\
\hline In percent of exports & $\ldots$ & $\ldots$ & 64.7 & & & 83.3 & 89.5 & 98.4 & 101.8 & 104.9 & 106.3 & & 99.2 & 76.5 & \\
\hline In percent of government revenues & $\ldots$ & $\ldots$ & 80.0 & & & 101.2 & 111.2 & 120.5 & 122.1 & 121.7 & 120.6 & & 106.5 & 86.0 & \\
\hline Debt service-to-exports ratio (in percent) & 18.4 & 34.7 & 12.3 & & & 16.4 & 16.4 & 16.9 & 16.1 & 16.6 & 14.5 & & 17.0 & 21.2 & \\
\hline PPG debt service-to-exports ratio (in percent) & 1.7 & 2.2 & 2.2 & & & 3.8 & 4.2 & 5.2 & 6.5 & 7.3 & 7.6 & & 7.6 & 5.7 & \\
\hline PPG debt service-to-revenue ratio (in percent) & 2.6 & 3.4 & 2.8 & & & 4.6 & 5.2 & 6.3 & 7.8 & 8.5 & 8.6 & & 8.1 & 6.4 & \\
\hline Total gross financing need (Billions of U.S. dollars) & 0.7 & 1.2 & -0.2 & & & 0.6 & 0.8 & 0.9 & 0.9 & 1.1 & 1.2 & & 3.2 & 11.3 & \\
\hline Non-interest current account deficit that stabilizes debt ratio & 12.8 & 10.1 & 25.5 & & & 8.6 & 11.3 & 10.3 & 11.1 & 10.3 & 9.8 & & 9.5 & 8.4 & \\
\hline Key macroeconomic assumptions & & & & & & & & & & & & & & & \\
\hline Real GDP growth (in percent) & 6.3 & 6.8 & 7.1 & 7.5 & 1.0 & 6.7 & 7.2 & 7.8 & 7.8 & 7.8 & 7.8 & 7.5 & 7.5 & 7.5 & 7.5 \\
\hline GDP deflator in US dollar terms (change in percent) & -5.7 & -10.9 & 25.7 & 4.8 & 11.0 & 4.2 & 1.6 & 3.9 & 3.8 & 3.8 & 3.6 & 3.5 & 2.6 & 2.1 & 2.8 \\
\hline Effective interest rate (percent) $5 /$ & 2.4 & 2.4 & 2.9 & 3.1 & 1.1 & 3.1 & 3.1 & 3.2 & 3.0 & 2.9 & 2.9 & 3.0 & 3.0 & 3.1 & 3.3 \\
\hline Growth of exports of G\&S (US dollar terms, in percent) & -14.0 & 8.0 & 15.9 & 14.5 & 13.4 & 10.9 & 13.2 & 11.9 & 11.2 & 9.9 & 9.8 & 11.1 & 10.5 & 11.7 & 11.3 \\
\hline Aid flows (in Billions of US dollars) 7/ & 0.9 & 0.9 & 1.0 & & & 1.4 & 1.5 & 1.7 & 1.9 & 2.1 & 2.3 & & 3.4 & 5.5 & \\
\hline o/w Grants & 0.9 & 0.9 & 1.0 & & & 0.9 & 1.0 & 1.1 & 1.2 & 1.3 & 1.5 & & 2.2 & 3.0 & \\
\hline o/w Concessional loans & 0.0 & 0.0 & 0.0 & & & 0.5 & 0.5 & 0.6 & 0.7 & 0.8 & 0.8 & & 1.2 & 2.4 & \\
\hline Grant-equivalent financing (in percent of GDP) 8/ & $\ldots$ & $\ldots$ & $\ldots$ & & & 8.7 & 8.1 & 8.0 & 7.8 & 7.7 & 7.7 & & 6.7 & 3.9 & 5.7 \\
\hline Grant-equivalent financing (in percent of external financing) 8/ & $\ldots$ & $\ldots$ & $\ldots$ & & & 63.4 & 59.5 & 59.5 & 62.3 & 64.9 & 66.5 & & 68.9 & 58.6 & 65.5 \\
\hline Memorandum items: & & & & & & & & & & & & & & & \\
\hline Nominal GDP (Billions of US dollars) & 10.0 & 9.5 & 12.8 & & & 14.2 & 15.5 & 17.3 & 19.4 & 21.7 & 24.2 & & 41.3 & 108.6 & \\
\hline Nominal dollar GDP growth & 0.2 & -4.9 & 34.6 & & & 11.3 & 8.9 & 12.0 & 12.0 & 11.9 & 11.7 & 11.3 & 10.3 & 9.7 & 10.5 \\
\hline PV of PPG external debt (in Billions of US dollars) & & & 2.4 & & & 3.1 & 3.9 & 4.7 & 5.5 & 6.1 & 6.8 & & 10.7 & 24.3 & \\
\hline (PVt-PVt-1)/GDPt-1 (in percent) & & & & & & 5.3 & 5.6 & 5.5 & 4.3 & 3.5 & 3.2 & 4.6 & 2.2 & 2.0 & 2.2 \\
\hline Gross workers' remittances (Billions of US dollars) & $\ldots$ & $\ldots$ & $\ldots$ & & & $\ldots$ & ... & $\ldots$ & $\ldots$ & $\ldots$ & $\ldots$ & & $\ldots$ & $\ldots$ & \\
\hline PV of PPG external debt (in percent of GDP + remittances) & $\ldots$ & $\ldots$ & 17.5 & & & 22.5 & 25.1 & 27.6 & 28.3 & 28.6 & 28.5 & & 26.2 & 22.7 & \\
\hline PV of PPG external debt (in percent of exports + remittances) & $\ldots$ & $\ldots$ & 64.7 & & & 83.3 & 89.5 & 98.4 & 101.8 & 104.9 & 106.3 & & 99.2 & 76.5 & \\
\hline Debt service of PPG external debt (in percent of exports + remitta & $\ldots$ & $\ldots$ & 2.2 & & & 3.8 & 4.2 & 5.2 & 6.5 & 7.3 & 7.6 & & 7.6 & 5.7 & \\
\hline
\end{tabular}

Sources: Country authorities; and staff estimates and projections.

$1 /$ Includes both public and private sector external debt.

2/ Derived as $[r-g-\rho(1+g)] /(1+g+\rho+g \rho)$ times previous period debt ratio, with $r=$ nominal interest rate; $g=$ real GDP growth rate, and $\rho=$ growth rate of GDP deflator in U.S. dollar terms.

$3 /$ Includes exceptional financing (i.e., changes in arrears and debt relief); changes in gross foreign assets; and valuation adjustments. For projections also includes contribution from price and exchange rate changes. 4/ Assumes that PV of private sector debt is equivalent to its face value.

$5 /$ Current-year interest payments divided by previous period debt stock.

6/ Historical averages and standard deviations are generally derived over the past 10 years, subject to data availability.

7/ Defined as grants, concessional loans, and debt relief.

8/ Grant-equivalent financing includes grants provided directly to the government and through new borrowing (difference between the face value and the PV of new debt). 
Table 2. Sensitivity Analysis for Key Indicators of Public and Publicly Guaranteed

External Debt, 2012-2032

(In percent)

PV of debt-to GDP ratio
Baseline
A. Alternative Scenarios 1/
A1. Key variables at their historical averages in 2012-2032 $2 /$
A3. Alternative scenario: High Investment/Low growth
B. Bound Tests
B1. Real GDP growth at historical average minus one standard deviation in 2013-2014
B2. Export value growth at historical average minus one standard deviation in 2013-2014 3/
B3. US dollar GDP deflator at historical average minus one standard deviation in 2013-2014
B4. Net non-debt creating flows at historical average minus one standard deviation in 2013-2014 4/
B5. Combination of B1-B4 using one-half standard deviation shocks
B6. One-time 30 percent nominal depreciation relative to the baseline in 2013 5/

201

\begin{tabular}{lll} 
& \multicolumn{3}{c}{ Projections } \\
\hline 2013 & 2014 & $2015 \quad 2016$
\end{tabular}

Projections
$\begin{array}{lllll}2015 & 2016 & 2017 & 2022 & 2032\end{array}$

$\begin{array}{llllllll}22 & 25 & 28 & 28 & 29 & 29 & \mathbf{2 6} & 23 \\ 22 & 27 & 31 & 35 & 36 & 38 & \mathbf{3 6} & 23 \\ 22 & 26 & 29 & 30 & 31 & 31 & 30 & 27\end{array}$

PV of debt-to-exports ratio

Baseline

83

A. Alternative Scenarios 1/

A1. Key variables at their historical averages in 2012-2032 2/

A3. Alternative scenario: High Investment/Low growth

83

$\begin{array}{rrrrrrrr}95 & 111 & 124 & 134 & 140 & \mathbf{1 3 7} & 76\end{array}$

B. Bound Tests

B1. Real GDP growth at historical average minus one standard deviation in 2013-2014 B2. Export value growth at historical average minus one standard deviation in 2013-2014 3/

B3. US dollar GDP deflator at historical average minus one standard deviation in 2013-2014

B4. Net non-debt creating flows at historical average minus one standard deviation in 2013-2014 4/

B5. Combination of B1-B4 using one-half standard deviation shocks

B6. One-time 30 percent nominal depreciation relative to the baseline in 2013 5/

PV of debt-to-revenue ratio

Baseline

A. Alternative Scenarios 1/

A1. Key variables at their historical averages in 2012-2032 2/

A3. Alternative scenario: High Investment/Low growth

\section{B. Bound Tests}

B1. Real GDP growth at historical average minus one standard deviation in 2013-2014

B2. Export value growth at historical average minus one standard deviation in 2013-2014 3/

B3. US dollar GDP deflator at historical average minus one standard deviation in 2013-2014

B4. Net non-debt creating flows at historical average minus one standard deviation in 2013-2014 4/

B5. Combination of B1-B4 using one-half standard deviation shocks

B6. One-time 30 percent nominal depreciation relative to the baseline in 20135 /

111

$\begin{array}{llllll}28 & 29 & 29 & 29 & \mathbf{2 6} & 23 \\ 33 & 34 & 33 & 33 & \mathbf{2 9} & 23 \\ 33 & 34 & 34 & 34 & \mathbf{3 1} & 27 \\ 38 & 38 & 38 & 37 & \mathbf{3 1} & 24 \\ 39 & 39 & 39 & 38 & \mathbf{3 2} & 25 \\ 39 & 40 & 40 & 40 & \mathbf{3 6} & 32\end{array}$

$\begin{array}{rrrrrrrr}101 & 118 & 136 & 149 & 155 & 159 & \mathbf{1 4 7} & 86 \\ 98 & 117 & 126 & 131 & 132 & 129 & 121 & 103\end{array}$

$\begin{array}{rrrrrrrr}101 & 111 & 121 & 123 & 122 & 121 & \mathbf{1 0 6} & 86 \\ 101 & 120 & 145 & 145 & 142 & 139 & \mathbf{1 1 6} & 87 \\ 101 & 120 & 142 & 145 & 144 & 143 & \mathbf{1 2 5} & 101 \\ 101 & 137 & 167 & 165 & 160 & 156 & \mathbf{1 2 6} & 89 \\ 101 & 133 & 171 & 170 & 165 & 161 & \mathbf{1 3 2} & 96 \\ 101 & 157 & 168 & 172 & 171 & 169 & \mathbf{1 4 8} & 120\end{array}$




\section{Table 2. Sensitivity Analysis for Key Indicators of Public and Publicly Guaranteed External Debt, 2012-2032 (continued)}

(In percent)

\section{Debt Service-to-exports ratio}

Baseline

A. Alternative Scenarios 1/

A1. Key variables at their historical averages in 2012-2032 2/

A3. Alternative scenario: High Investment/Low growth

B. Bound Tests

B1. Real GDP growth at historical average minus one standard deviation in 2013-2014 B2. Export value growth at historical average minus one standard deviation in 2013-2014 3/ B3. US dollar GDP deflator at historical average minus one standard deviation in 2013-2014 B4. Net non-debt creating flows at historical average minus one standard deviation in 2013-2014 4/ B5. Combination of B1-B4 using one-half standard deviation shocks

B6. One-time 30 percent nominal depreciation relative to the baseline in 20135 /

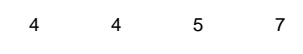

$\begin{array}{ll}4 & 4 \\ 4 & 4\end{array}$

$\begin{array}{rrrrrr}5 & 7 & 8 & 8 & 9 & 6 \\ 5 & 7 & 7 & 8 & 9 & 10\end{array}$

Debt service-to-revenue ratio

\section{Baseline}

A. Alternative Scenarios 1/

A1. Key variables at their historical averages in 2012-2032 2/

A3. Alternative scenario: High Investment/Low growth

B. Bound Tests

B1. Real GDP growth at historical average minus one standard deviation in 2013-2014 B2. Export value growth at historical average minus one standard deviation in 2013-2014 3/

B3. US dollar GDP deflator at historical average minus one standard deviation in 2013-2014

B4. Net non-debt creating flows at historical average minus one standard deviation in 2013-2014 4/

B5. Combination of B1-B4 using one-half standard deviation shocks

B6. One-time 30 percent nominal depreciation relative to the baseline in 20135 /

Memorandum item:

Grant element assumed on residual financing (i.e., financing required above baseline) $6 /$

Sources: Country authorities; and staff estimates and projections.

1/ The stress test A2 (borrowing in less concessional terms) has not been included in this case (in line with the 2010 and 2011 DSAs). Given the commercial financing terms already included in the baseline for the investment projects, this scenario would yield unrealistic results, as marginal borrowing under this shock is calculated on the average terms of new borrowing; therefore assuming harsher terms than Mozambique would actually face in the need to cover a financing gap.

2/ Variables include real GDP growth, growth of GDP deflator (in U.S. dollar terms), non-interest current account in percent of GDP, and non-debt creating flows

3/ Exports values are assumed to remain permanently at the lower level, but the current account as a share of GDP is assumed to return to its baseline level after the shock (implicitly assuming an offsetting adjustment in import levels).

4 / Includes official and private transfers and FDI.

$5 /$ Depreciation is defined as percentage decline in dollar/local currency rate, such that it never exceeds 100 percent.

6/ Applies to all stress scenarios except for A2 (less favorable financing) in which the terms on all new financing are as specified in footnote 2. 
Figure 2. Mozambique: Indicators of Public of Public Debt Under Alternative Scenarios, 2012-2032 ${ }^{1}$
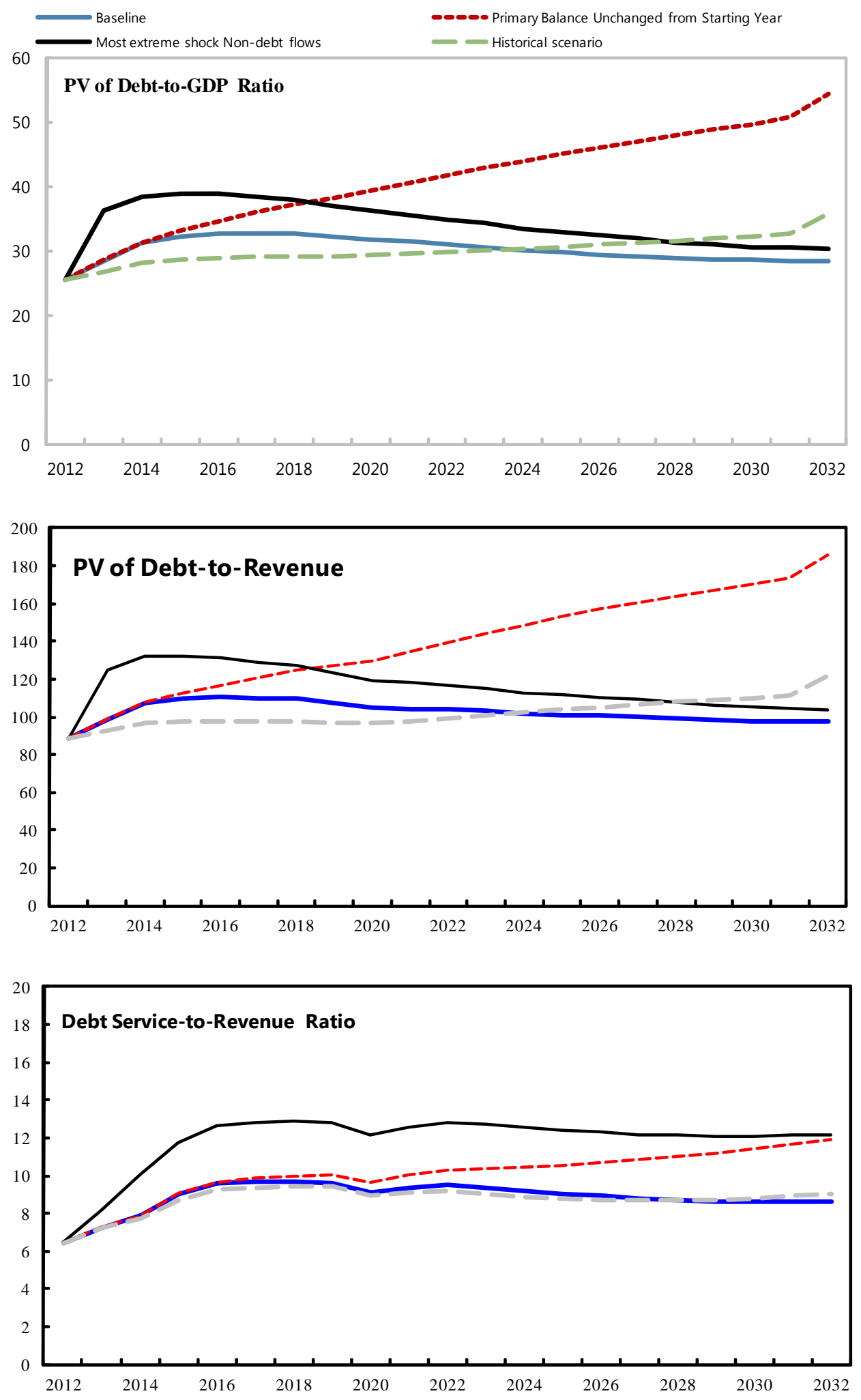

Sources: Country authorities; and staff estimates and projections.

$1 /$ The most extreme stress test is the test that yields the highest ratio in 2022.

2/ Revenues are defined inclusive of grants. 
Table 3a. Mozambique: Public Sector Debt Sustainability Framework, Baseline Scenario, 2009-2032

(In percent of GDP, unless otherwise indicated)

\begin{tabular}{|c|c|c|c|c|c|c|c|c|c|c|c|c|c|c|c|}
\hline & \multicolumn{3}{|c|}{ Actual } & \multirow[b]{2}{*}{ Average ${ }^{5 /}$} & \multirow[b]{2}{*}{$\begin{array}{l}\text { Standard } \\
\text { Deviation }\end{array}$} & \multicolumn{4}{|l|}{ Estimate } & \multicolumn{3}{|c|}{ Projections } & \multirow{2}{*}{\multicolumn{3}{|c|}{$\begin{array}{c}2018- \\
32 \\
2032 \text { Average }\end{array}$}} \\
\hline & 2009 & 2010 & 2011 & & & 2012 & 2013 & 2014 & 2015 & 2016 & 2017 & $\begin{array}{l}2012-17 \\
\text { Average }\end{array}$ & & & \\
\hline $\begin{array}{l}\text { Public sector debt } 1 / \\
\text { o/w foreign-currency denominated }\end{array}$ & $\begin{array}{l}41.6 \\
38.4\end{array}$ & $\begin{array}{l}41.4 \\
39.0\end{array}$ & $\begin{array}{l}34.4 \\
31.5\end{array}$ & & & $\begin{array}{l}41.4 \\
38.3\end{array}$ & $\begin{array}{l}43.9 \\
40.6\end{array}$ & $\begin{array}{l}46.7 \\
43.1\end{array}$ & $\begin{array}{l}47.2 \\
43.4\end{array}$ & $\begin{array}{l}47.5 \\
43.5\end{array}$ & $\begin{array}{l}47.3 \\
43.1\end{array}$ & & $\begin{array}{l}44.4 \\
39.6\end{array}$ & $\begin{array}{l}39.1 \\
33.4\end{array}$ & \\
\hline Change in public sector debt & -0.5 & -0.2 & -7.0 & & & 7.0 & 2.6 & 2.8 & 0.5 & 0.3 & -0.2 & & -0.5 & -0.3 & \\
\hline Identified debt-creating flows & 4.5 & 3.9 & -7.3 & & & 6.1 & 1.9 & 2.2 & 0.3 & 0.4 & -0.1 & & -0.4 & -3.6 & \\
\hline Primary deficit & 5.0 & 3.1 & 4.0 & 3.1 & 1.0 & 5.2 & 5.2 & 5.2 & 4.2 & 3.8 & 3.4 & 4.5 & 2.5 & -1.3 & 2.2 \\
\hline Revenue and grants & 27.1 & 29.7 & 29.6 & & & 28.8 & 28.9 & 29.0 & 29.3 & 29.6 & 29.8 & & 29.8 & 29.2 & \\
\hline of which: grants & 9.5 & 9.1 & 7.7 & & & 6.6 & 6.3 & 6.1 & 6.1 & 6.1 & 6.1 & & 5.2 & 2.8 & \\
\hline Primary (noninterest) expenditure & 32.1 & 32.8 & 33.6 & & & 34.0 & 34.2 & 34.2 & 33.5 & 33.4 & 33.2 & & 32.3 & 27.8 & \\
\hline Automatic debt dynamics & -0.3 & 1.0 & -11.3 & & & 0.8 & -3.4 & -3.0 & -3.9 & -3.4 & -3.5 & & -2.9 & -2.3 & \\
\hline Contribution from interest rate/growth differential & 0.0 & 2.3 & -9.5 & & & -2.4 & -2.2 & -3.3 & -3.6 & -3.6 & -3.5 & & -2.9 & -2.3 & \\
\hline of which: contribution from average real interest rate & 2.5 & 5.0 & -6.7 & & & -0.2 & 0.6 & -0.2 & -0.2 & -0.2 & -0.1 & & 0.3 & 0.4 & \\
\hline of which: contribution from real GDP growth & -2.5 & -2.6 & -2.8 & & & -2.2 & -2.8 & -3.2 & -3.4 & -3.4 & -3.4 & & -3.1 & -2.7 & \\
\hline Contribution from real exchange rate depreciation & -0.3 & -1.4 & -1.8 & & & 3.2 & -1.2 & 0.4 & -0.3 & 0.2 & 0.0 & & $\ldots$ & & \\
\hline Other identified debt-creating flows & -0.3 & -0.2 & 0.0 & & & 0.0 & 0.0 & 0.0 & 0.0 & 0.0 & 0.0 & & 0.0 & 0.0 & \\
\hline Privatization receipts (negative) & -0.2 & -0.1 & 0.0 & & & 0.0 & 0.0 & 0.0 & 0.0 & 0.0 & 0.0 & & 0.0 & 0.0 & \\
\hline Recognition of implicit or contingent liabilities & 0.0 & 0.0 & 0.0 & & & 0.0 & 0.0 & 0.0 & 0.0 & 0.0 & 0.0 & & 0.0 & 0.0 & \\
\hline Debt relief (HIPC and other) & -0.1 & -0.1 & 0.0 & & & 0.0 & 0.0 & 0.0 & 0.0 & 0.0 & 0.0 & & 0.0 & 0.0 & \\
\hline Other (specify, e.g. bank recapitalization) & 0.0 & 0.0 & 0.0 & & & 0.0 & 0.0 & 0.0 & 0.0 & 0.0 & 0.0 & & 0.0 & 0.0 & \\
\hline Residual, including asset changes & -5.0 & -4.1 & 0.2 & & & 0.9 & 0.7 & 0.5 & 0.3 & -0.1 & -0.1 & & -0.2 & 3.4 & \\
\hline \multicolumn{16}{|l|}{ Other Sustainability Indicators } \\
\hline $\mathrm{PV}$ of public sector debt & $\ldots$ & $\ldots$ & 20.4 & & & 25.6 & 28.5 & 31.2 & 32.1 & 32.6 & 32.7 & & 31.0 & 28.3 & \\
\hline $\mathrm{o} / \mathrm{w}$ foreign-currency denominated & $\ldots$ & $\ldots$ & 17.5 & & & 22.5 & 25.1 & 27.6 & 28.3 & 28.6 & 28.5 & & 26.2 & 22.7 & \\
\hline $\mathrm{o} / \mathrm{w}$ external & $\ldots$ & $\ldots$ & 17.5 & & & 22.5 & 25.1 & 27.6 & 28.3 & 28.6 & 28.5 & & 26.2 & 22.7 & \\
\hline PV of contingent liabilities (not included in public sector debt) & $\ldots$ & $\ldots$ & $\ldots$ & & & $\ldots$ & & & & & & & & & \\
\hline Gross financing need 2/ & 5.9 & 4.7 & 5.4 & & & 7.1 & 7.3 & 7.5 & 6.8 & 6.6 & 6.3 & & 5.3 & 1.2 & \\
\hline $\mathrm{PV}$ of public sector debt-to-revenue and grants ratio (in percent) & $\cdots$ & $\ldots$ & 68.9 & & & 88.8 & 98.4 & 107.4 & 109.7 & 110.4 & 109.9 & & 103.9 & 97.2 & \\
\hline $\mathrm{PV}$ of public sector debt-to-revenue ratio (in percent) & $\ldots$ & $\ldots$ & 93.3 & & & 115.2 & 126.0 & 136.2 & 138.6 & 138.9 & 138.4 & & 126.1 & 107.5 & \\
\hline o/w external $3 /$ & & & 80.0 & & & 101.2 & 111.2 & 120.5 & 122.1 & 121.7 & 120.6 & & 106.5 & 86.0 & \\
\hline Debt service-to-revenue and grants ratio (in percent) $4 /$ & 3.5 & 5.1 & 4.6 & & & 6.5 & 7.3 & 7.9 & 9.0 & 9.6 & 9.7 & & 9.5 & 8.6 & \\
\hline Debt service-to-revenue ratio (in percent) 4/ & 5.4 & 7.4 & 6.3 & & & 8.4 & 9.3 & 10.0 & 11.4 & 12.1 & 12.2 & & 11.5 & 9.5 & \\
\hline Primary deficit that stabilizes the debt-to-GDP ratio & 5.5 & 3.3 & 11.1 & & & -1.8 & 2.6 & 2.4 & 3.6 & 3.5 & 3.6 & & 3.0 & -1.0 & \\
\hline \multicolumn{16}{|l|}{ Key macroeconomic a } \\
\hline Real GDP growth (in percent) & 6.3 & 6.8 & 7.1 & 7.5 & 1.0 & 6.7 & 7.2 & 7.8 & 7.8 & 7.8 & 7.8 & 7.5 & 7.5 & 7.5 & 7.5 \\
\hline Average nominal interest rate on forex debt (in percent) & 0.6 & 0.6 & 0.7 & 0.6 & 0.3 & 1.2 & 1.4 & 1.6 & 1.7 & 1.8 & 1.8 & 1.6 & 1.6 & $1.6^{\prime \prime}$ & 1.6 \\
\hline Average real interest rate on domestic debt (in percent) & 3.9 & 10.5 & 22.0 & 12.5 & 16.0 & 23.7 & 23.1 & 21.1 & 19.3 & 18.0 & $16.8^{\prime \prime}$ & 20.3 & 13.8 & $11.0^{\circ}$ & 12.7 \\
\hline Real exchange rate depreciation (in percent, + indicates depreciat & -0.8 & -3.4 & -6.2 & -1.2 & 12.0 & 11.3 & & & & & & & & & \\
\hline Inflation rate (GDP deflator, in percent) & 4.2 & 10.0 & 10.7 & 7.9 & 2.1 & 7.6 & 6.0 & 6.0 & 6.0 & 5.9 & 5.8 & 6.2 & 5.8 & 5.2 & 5.7 \\
\hline Growth of real primary spending (deflated by GDP deflator, in perc & 0.3 & 0.1 & 0.1 & 0.1 & 0.1 & 0.1 & 0.1 & 0.1 & 0.1 & 0.1 & 0.1 & 0.1 & 0.1 & 0.0 & 0.1 \\
\hline Grant element of new external borrowing (in percent) & $\ldots$ & $\ldots$ & $\ldots$ & $\ldots$ & $\ldots$ & 29.8 & 24.4 & 25.2 & 26.2 & 28.1 & 29.0 & 27.1 & 32.2 & 28.5 & \\
\hline
\end{tabular}

Sources: Country authorities; and staff estimates and projections.

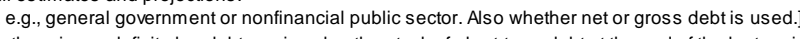

作

hebt service is definedas.

$5 /$ Historical averages and standard deviations are generally derived over the past 10 years, subject to data availability. 
Table 3b. Mozambique: Sensitivity Analysis for Key Indicators of Public Debt, 2012-2032

\begin{tabular}{|c|c|c|c|c|c|c|c|c|}
\hline & \multicolumn{8}{|c|}{ Projections } \\
\hline & 2012 & 2013 & 2014 & 2015 & 2016 & 2017 & 2022 & 2032 \\
\hline \multicolumn{9}{|l|}{ PV of Debt-to-GDP Ratio } \\
\hline Baseline & 26 & 28 & 31 & 32 & 33 & 33 & 31 & 28 \\
\hline \multicolumn{9}{|l|}{ A. Alternative scenarios } \\
\hline A1. Real GDP growth and primary balance are at his torical averages & 26 & 27 & 28 & 29 & 29 & 29 & 30 & 36 \\
\hline A2. Primary balance is unchanged from 2012 & 26 & 28 & 31 & 33 & 35 & 36 & 42 & 54 \\
\hline A3. Permanently lower GDP growth 1/ & 26 & 29 & 31 & 33 & 33 & 34 & 33 & 35 \\
\hline \multicolumn{9}{|l|}{ B. Bound tests } \\
\hline B1. Real GDP growth is at historical average minus one standard deviations in $2013-2014$ & 26 & 29 & 32 & 33 & 34 & 35 & 34 & 33 \\
\hline B2. Primary balance is at historical average minus one standard deviations in $2013-2014$ & 26 & 28 & 29 & 31 & 31 & 31 & 30 & 28 \\
\hline B3. Combination of B1-B2 using one half standard deviation shocks & 26 & 27 & 29 & 30 & 31 & 32 & 31 & 30 \\
\hline B4. One-time 30 percent real depreciation in 2013 & 26 & 37 & 38 & 38 & 38 & 37 & 33 & 31 \\
\hline B5. 10 percent of GDP increase in other debt-creating flows in 2013 & 26 & 36 & 38 & 39 & 39 & 38 & 35 & 30 \\
\hline
\end{tabular}

PV of Debt-to-Revenue Ratio 2/

Baseline

$\begin{array}{llllllll}89 & 98 & 107 & 110 & 110 & 110 & 104 & 97\end{array}$

A. Alternative scenarios

A1. Real GDP growth and primary balance are at historical averages

A2. Primary balance is unchanged from 2012

A3. Permanently lower GDP growth $1 /$

$\begin{array}{rrrrrrrr}89 & 92 & 97 & 97 & 97 & 97 & 99 & 122 \\ 89 & 98 & 108 & 113 & 117 & 121 & 140 & 186\end{array}$

\section{B. Bound tests}

B1. Real GDP growth is at historical average minus one standard deviations in 2013-2014

B2. Primary balance is at historical average minus one standard deviations in 2013-2014

B3. Combination of B1-B2 using one half standard deviation shocks

B4. One-time 30 percent real depreciation in 2013

B5. 10 percent of GDP increase in other debt-creating flows in 2013

Debt Service-to-Revenue Ratio 2/

Baseline

$\begin{array}{rrrrrrrr}89 & 99 & 110 & 114 & 115 & 116 & 113 & 112 \\ 89 & 95 & 102 & 104 & 106 & 105 & 101 & 96 \\ 89 & 94 & 100 & 103 & 105 & 106 & 104 & 102 \\ 89 & 127 & 132 & 130 & 127 & 124 & 112 & 106 \\ 89 & 125 & 132 & 132 & 131 & 129 & 117 & 104\end{array}$

\section{A. Alternative scenarios}

A1. Real GDP growth and primary balance are at historical averages

A2. Primary balance is unchanged from 2012

A3. Permanently lower GDP growth $1 /$

$\begin{array}{rrrrrrrr}6 & 7 & 8 & 9 & 9 & 9 & 9 & 9 \\ 6 & 7 & 8 & 9 & 10 & 10 & 10 & 12 \\ 6 & 7 & 8 & 9 & 10 & 10 & 10 & 10\end{array}$

\section{B. Bound tests}

B1. Real GDP growth is at historical average minus one standard deviations in 2013-2014 B2. Primary balance is at historical average minus one standard deviations in 2013-2014

B3. Combination of B1-B2 using one half standard deviation shocks

B4. One-time 30 percent real depreciation in 2013

B5. 10 percent of GDP increase in other debt-creating flows in 2013

\begin{tabular}{rrrrrrrr}
6 & 7 & 8 & 9 & 10 & 10 & 10 & 9 \\
6 & 7 & 8 & 9 & 9 & 10 & 9 & 8 \\
6 & 7 & 8 & 9 & 9 & 10 & 9 & 9 \\
6 & 8 & 10 & 12 & 13 & 13 & 13 & 12 \\
6 & 7 & 9 & 10 & 10 & 10 & 10 & 9 \\
\hline
\end{tabular}

Sources: Country authorities; and staff estimates and projections.

1 / Assumes that real GDP growth is at baseline minus one standard deviation divided by the square root of the length of the projection period.

2/ Revenues are defined inclusive of grants. 


\title{
INTERNATIONAL MONETARY FUND
}

\section{REPUBLIC OF MOZAMBIQUE}

FOURTH REVIEW UNDER THE POLICY SUPPORT

May 11, 2012 INSTRUMENT AND REQUEST FOR MODIFICATION OF ASSESSMENT CRITERIA-INFORMATIONAL ANNEX

\author{
Prepared By \\ African Department
}

\section{CONTENTS}

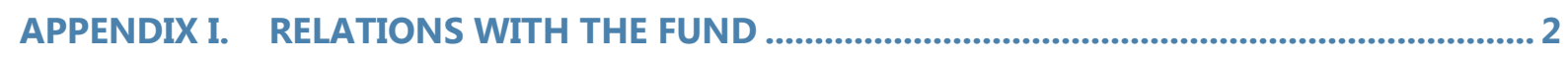

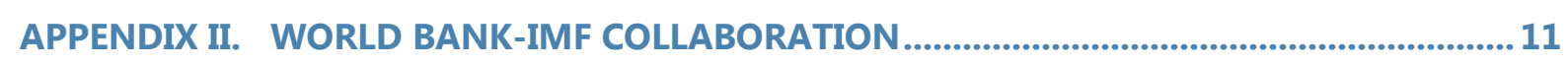

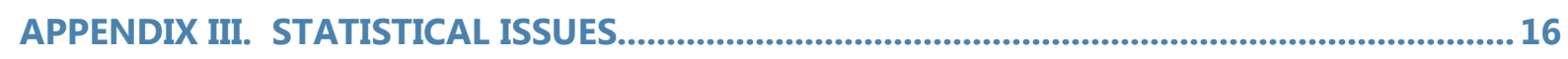




\title{
APPENDIX I. RELATIONS WITH THE FUND
}

\author{
(As of March 31, 2012)
}

I. Membership Status: Joined: September 24, 1984; Article VIII

II. General Resources Account:

Quota

Fund holdings of currency (Exchange Rate)

Reserve Tranche Position

\section{SDR Department:}

Net cumulative allocation

Holdings

$\begin{array}{rr}\text { SDR Million } & \text { \%Quota } \\ 113.60 & 100.00 \\ 113.60 & 100.00 \\ 0.0 .1 & 0.0 .1\end{array}$

SDR Million

108.84

107.02

SDR Million

113.60

\%Allocation

100.00

8.12

7.15

V. Latest Financial Arrangements:

Date of Expiration Amount Approved Amount Drawn

\begin{tabular}{lccrr}
\multicolumn{1}{r}{ Type } & Arrangement & Date & (SDR Million) & (SDR Million) \\
ESF & Jun 30, 2009 & Jun 28, 2010 & 113.60 & 113.60 \\
& & & & \\
ECF $^{1}$ & Jul 06, 2004 & Jul 05, 2007 & 11.36 & 11.36 \\
ECF & Jun 28, 1999 & Jun 28, 2003 & 87.20 & 78.80
\end{tabular}

\footnotetext{
${ }^{1}$ Former PRGF.
} 


\section{Projected Payments to Fund ${ }^{2}$}

(SDR Million; based on existing use of resources and present holdings of SDRs):

\begin{tabular}{|c|c|c|c|c|c|}
\hline & \multicolumn{5}{|c|}{ Forthcoming } \\
\hline & $\underline{2012}$ & $\underline{2013}$ & $\underline{2014}$ & $\underline{2015}$ & $\underline{2016}$ \\
\hline Principal & 0.97 & 1.95 & 1.95 & 23.09 & 23.70 \\
\hline $\begin{array}{l}\text { Charges/ } \\
\text { Interest }\end{array}$ & 0.00 & 0.29 & 0.30 & 0.26 & 0.20 \\
\hline Total & $\underline{0.98}$ & $\underline{2.24}$ & $\underline{2.25}$ & 23.35 & $\underline{23.90}$ \\
\hline
\end{tabular}

\section{Implementation of HIPC Initiative:}

I. Commitment of HIPC assistance

\begin{tabular}{rrr}
$\begin{array}{rr}\text { Original } \\
\text { Framework }\end{array}$ & $\begin{array}{r}\text { Enhanced } \\
\text { Framework }\end{array}$ \\
\cline { 1 - 1 } Apr 1998 & & Apr 2000 \\
& \\
$1,716.00$ & 306.00 \\
124.60 & 18.46 \\
93.17 & 13.73
\end{tabular}

Completion point date

Jun. 1999 Sep. 2001

II. Disbursement of IMF assistance (SDR Million)

Assistance disbursed to the member

13.73

106.90

Interim assistance

2.31

2.31

Completion point balance

93.17

11.42

104.58

Additional disbursement of interest income ${ }^{4}$

1.10

1.10

Total disbursements

\footnotetext{
${ }^{2}$ When a member has overdue financial obligations outstanding for more than three months, the amount of such arrears will be shown in this section.

${ }^{3}$ Assistance committed under the original framework is expressed in net present value (NPV) terms at the completion point, and assistance committed under the enhanced framework is expressed in NPV terms at the decision point. Hence these two amounts cannot be added.

${ }^{4}$ Under the enhanced framework, an additional disbursement is made at the completion point corresponding to interest income earned on the amount committed at the decision point but not disbursed during the interim period.
} 


\section{Implementation of Multilateral Debt Relief Initiative (MDRI):}

I. MDRI-eligible debt (SDR Million) ${ }^{5}$

106.56

Financed by: MDRI Trust $\quad 83.05$

$\begin{array}{ll}\text { Remaining HIPC resources } & 23.51\end{array}$

II. Debt Relief by Facility (SDR Million)

\begin{tabular}{lrrr} 
& \multicolumn{3}{c}{ Eligible Debt } \\
\cline { 2 - 4 } Delivery Date & $\underline{\text { GRA }}$ & $\underline{\text { PRGF }}$ & $\underline{\underline{\text { Total }}}$ \\
January 2006 & N/A & 106.56 & 106.56
\end{tabular}

IX. Implementation of Post-Catastrophe Debt Relief (PCDR): Not Applicable

\section{Safeguards assessment}

An update safeguards assessment of the Bank of Mozambique (BM) was completed on January 11, 2010 for the ESF. The assessment found improvements in financial reporting practices following the implementation of IFRS. It noted vulnerabilities in the oversight mechanism for external and internal auditing, controls, financial reporting, and in the BM's legal structure. It recommended that the BM commission an external quality assurance review of the internal audit function.

\section{Exchange Rate Arrangement}

Mozambique has a de jure and de facto floating exchange rate arrangement. The exchange rate is largely determined in the interbank foreign exchange market (MCI). The BM regularly intervenes in the market to smooth seasonal fluctuations, provide foreign exchange liquidity received by the government in the form of aid, and sterilize domestic liquidity as part of its monetary policy operations.

On May 20, 2011, Mozambique accepted its obligations under Article VIII, Sections 2, 3, and 4 of the Fund's Articles of Agreement (Country Report No. 11/149). A new foreign exchange law_"Foreign Exchange Law" Law No. 11/2009_came into effect on March 11, 2009. A new foreign exchange regulation to implement the foreign exchange law-the "Regulation for the Foreign Exchange Law" (the "Regulation") (Decreto No. 83/2010)—was issued on December 31, 2010. The Regulation, in conjunction with the implementing norms subsequently issued by the $\mathrm{BM}$, fully removed the existing exchange restrictions subject to Article VIII, Sections 2, 3, and 4 of the Fund's Articles of Agreement as of July 11,2011 . The two existing multiple currency practices (MCP) were also removed. The MCP arising from the foreign exchange auction system was removed on April 27, 2011 through the adoption of a new regulation on the interbank exchange market and that discontinued the previous multiple price foreign exchange auction system, which in any case had not been used since 2009 . The

\footnotetext{
${ }^{5}$ The MDRI provides 100 percent debt relief to eligible member countries qualified for the assistance. Grant assistance from the MDRI Trust and HIPC resources provide debt relief to cover the full stock of debt owed to the Fund as of end-2004 outstanding at the time the member qualifies for debt relief.
} 
MCP arising from the absence of a mechanism to prevent potential deviations between the rate used by the BM in its foreign exchange transactions in the interbank foreign exchange market and in its transactions with the government, public entities and the World Bank was removed on July 1, 2011 through the adoption of a new mechanism for the determination of the exchange rate for such transactions of which the relevant parties have been notified on August 11, 2011.

\section{AML/CFT framework}

Mozambique's first AML/CFT Mutual Evaluation Report by the Eastern and Southern Africa AntiMoney Laundering Group (ESAAMLG) has been formally adopted by ESAAMLG and published on its website. Based on the deficiencies identified in the report, the Financial Action Task Force's (FATF) International Co-operation Review Group (ICRG) maintains Mozambique in a pool of jurisdictions which may be taken up for review. This process is designed to publicly identify high-risk jurisdictions, and could ultimately result in a public statement by the FATF to apply counter-measures. In order to improve compliance with the FATF Recommendations, the Financial Intelligence Unit (GIFIM) has begun its operations and is working towards membership of the Egmont Group of Financial Intelligence Units. However, the number of suspicious transaction reports (STRs) still appears to be low. Once the AML/CFT law is approved by Parliament, the BM intends to issue implementing guidelines. In order to ensure a consistent application of the AML/CFT framework across various regulators, the Government has established and appointed the members of the National AML/CFT Task Force in December 2011. The AML/CFT strategic plan has been approved by the Council of Ministers and will be submitted to ESAAMLG.

\section{Article IV Consultation}

In accordance with Decision No 12794-(02/76), as amended by Decision No 12854-(02/96), Mozambique is on a 24-month Article IV cycle due to the approval of the successor PSI in June 2010. The 2011 Article IV consultation was completed by the Executive Board on June 17, 2011 (Country Report No. 11/149).

In concluding the 2011 Article IV consultation, Executive Directors commended the authorities for their prudent policies that have resulted in a strong economic performance. They noted that, although Mozambique's medium-term prospects continue to be favorable, significant challenges remain in managing macroeconomic risks and ensuring that the benefits from growth are shared fairly across the population. In this regard, Directors underscored that safeguarding price stability and containing inflation expectations remain key policy priorities for the period ahead. They welcomed the authorities' new Poverty Reduction Strategy (PARP), including its focus on continued structural reforms to improve productivity and production in labor-intensive sectors such as agriculture, create jobs through improvements in the business environment, and pursue social and human development. These focus areas will be important to achieve sustained growth and poverty reduction. Directors supported the authorities' prudent fiscal stance aimed at preserving macroeconomic stability and debt sustainability, and encouraged the authorities to adopt a prudent approach to generating additional budgetary revenue from the natural resource sector while protecting Mozambique as a destination for foreign investment. Directors emphasized the importance of adhering to a prudent borrowing strategy, further strengthening debt management, and improving investment planning. They noted the resilience of the banking system during the global crisis, and recommended continued vigilance in light of remaining vulnerabilities.

\section{Ex post assessment of performance under Fund-supported programs}


An ex post assessment (EPA) of Mozambique's performance under Fund-Supported Programs since 1987 was undertaken in December 2003 under the guidelines on assessments of countries with a longer-term program engagement. The EPA was circulated to the Executive Board on November 21, 2003 (Country Report No. 04/53).

Directors commended the authorities for their pursuit of sound macroeconomic policies and wide ranging structural reforms over the past fifteen years which contributed to strong growth of the economy, and a steady decline in poverty rates. Noting that despite considerable progress Mozambique remains a very poor and vulnerable country, Directors urged the authorities to persevere in their efforts to consolidate macroeconomic stability and accelerate and deepen structural reforms with a view to sustaining economic growth, encouraging employment creation, and further reducing poverty.

\section{FSAP participation and ROSCs}

A Financial Sector Assessment Program (FSAP) for Mozambique was undertaken during the first quarter of 2003. The related Financial Sector Stability Assessment was circulated to the Executive Board on November 19, 2003 (Country Report No. 04/52). An update to the FSAP took place in February 2009 and the related Financial Sector Stability Assessment circulated to the Board on June 19, 2009 (Country Report No. 10/12). A ROSC on fiscal transparency was issued on February 22, 2001. This ROSC was updated in the context of the 2002 Article IV consultation (Country Report No. 02/140) and the 2003 Article IV Consultation (Country Report No. 04/50). The ROSC on fiscal transparency was updated in May 2008. A Report on the Observance of Standards and Codes (ROSC) data module was prepared in June 2002 and issued on March 5, 2003. This data module was updated in August 2005.

\section{Management's visit}

At the invitation of the authorities, Mr. Kato, Deputy Managing Director, visited Maputo, Mozambique in July 2005. The Managing Director visited Mozambique on August 2007 for a meeting with the African Consultative Group on Quotas, Voice, and Representation.

\section{Resident representative}

Mr. Victor Lledo has been the IMF's resident representative to Mozambique since June 21, 2010. 
XVIII. Technical Assistance (2005-2012)

\begin{tabular}{|c|c|c|c|c|}
\hline \multicolumn{5}{|c|}{$\begin{array}{l}\text { IMF Technical Assistance Provided to Mozambique (2005-12) } \\
\text { (As of April 16, 2012) }\end{array}$} \\
\hline Departments & Timing & Form & Purpose & Counterparts \\
\hline Finance & February 2008 & Mission & $\begin{array}{l}\text { Update of Safeguard } \\
\text { Assessment }\end{array}$ & $\begin{array}{l}\text { Bank of } \\
\text { Mozambique }\end{array}$ \\
\hline \multirow[t]{18}{*}{ Fiscal Affairs } & April 2012 & Mission & $\begin{array}{l}\text { Fiscal Regime of the } \\
\text { Extractive Sector }\end{array}$ & $\begin{array}{l}\text { Ministry of Finance } \\
\text { Ministry of Mineral } \\
\text { Resources }\end{array}$ \\
\hline & $\begin{array}{l}\text { March-April } \\
2102\end{array}$ & Peripatetic expert & Public Accounting & Ministry of Finance \\
\hline & $\begin{array}{l}\text { March-April } \\
2102\end{array}$ & Mission & $\begin{array}{l}\text { Public Financial Management } \\
\text { Reforms }\end{array}$ & Ministry of Finance \\
\hline & November 2011 & Mission & Tax Administration & Ministry of Finance \\
\hline & September 2011 & Peripatetic expert & Public Accounting & Ministry of Finance \\
\hline & September 2011 & Peripatetic expert & Tax Administration & Ministry of Finance \\
\hline & April 2011 & Peripatetic expert & Tax Administration & Ministry of Finance \\
\hline & April 2011 & Mission & $\begin{array}{l}\text { Follow-Up on Revenue } \\
\text { Administration }\end{array}$ & Ministry of Finance \\
\hline & March 2011 & Mission & $\begin{array}{l}\text { Public Financial Management } \\
\text { Reforms }\end{array}$ & Ministry of Finance \\
\hline & March 2011 & Peripatetic expert & Public Accounting & Ministry of Finance \\
\hline & November 2010 & Peripatetic expert & $\begin{array}{l}\text { Public Financial Management } \\
\text { Reforms }\end{array}$ & Ministry of Finance \\
\hline & April-May 2010 & Mission & $\begin{array}{l}\text { Revenue Administration } \\
\text { Reform }\end{array}$ & Ministry of Finance \\
\hline & April 2010 & Mission & $\begin{array}{l}\text { Public Financial Management } \\
\text { Reforms }\end{array}$ & Ministry of Finance \\
\hline & Feb.-Mar. 2010 & Mission & Tax Policy Review & Ministry of Finance \\
\hline & May 2009 & Mission & Tax Administration Reform & Ministry of Finance \\
\hline & April 2008 & Mission & $\begin{array}{l}\text { Public Financial Management } \\
\text { Reforms }\end{array}$ & Ministry of Finance \\
\hline & April-May 2008 & Mission & $\begin{array}{l}\text { Revenue Administration } \\
\text { Reform }\end{array}$ & Ministry of Finance \\
\hline & Oct.-Nov. 2007 & Mission & Tax Administration Inspection & Ministry of Finance \\
\hline
\end{tabular}




\begin{tabular}{|c|c|c|c|c|}
\hline \multicolumn{5}{|c|}{$\begin{array}{l}\text { IMF Technical Assistance Provided to Mozambique (2005-12) } \\
\text { (As of April 16, 2012) }\end{array}$} \\
\hline \multirow[t]{8}{*}{ Departments } & Timing & Form & Purpose & Counterparts \\
\hline & September 2007 & Mission & $\begin{array}{l}\text { Public Private Partnerships } \\
\text { and Fiscal Risks }\end{array}$ & Ministry of Finance \\
\hline & August 2007 & Peripatetic expert & Tax Policy and Administration & Ministry of Finance \\
\hline & August 2007 & Mission & $\begin{array}{l}\text { Fiscal ROSC Update and } \\
\text { Public Financial Management } \\
\text { inspection }\end{array}$ & Ministry of Finance \\
\hline & April-May 2007 & Mission & Tax Administration Inspection & Ministry of Finance \\
\hline & April-May 2007 & Mission & Petroleum Fiscal Regime & $\begin{array}{l}\text { Ministry of Finance } \\
\text { Ministry of Mineral } \\
\text { Resources }\end{array}$ \\
\hline & December 2006 & Mission & Tax Administration & Ministry of Finance \\
\hline & March 2006 & Mission & Tax Policy Review & Ministry of Finance \\
\hline \multirow[t]{9}{*}{$\begin{array}{l}\text { Monetary and } \\
\text { Financial Sectors }\end{array}$} & May 2012 & Short-term Expert & Monetary Policy Framework & $\begin{array}{l}\text { Bank of } \\
\text { Mozambique }\end{array}$ \\
\hline & April 2012 & Short-term Expert & Stress testing & $\begin{array}{l}\text { Bank of } \\
\text { Mozambique }\end{array}$ \\
\hline & February 2012 & Short-term Expert & $\begin{array}{l}\text { Developing a payment system } \\
\text { oversight function }\end{array}$ & $\begin{array}{l}\text { Bank of } \\
\text { Mozambique }\end{array}$ \\
\hline & December 2011 & Short-term Expert & $\begin{array}{l}\text { Developing a payment system } \\
\text { oversight function }\end{array}$ & $\begin{array}{l}\text { Bank of } \\
\text { Mozambique }\end{array}$ \\
\hline & September 2011 & Mission & $\begin{array}{l}\text { Follow-up on Medium-Term } \\
\text { Debt Strategy and Capacity to } \\
\text { Conduct Debt and Project } \\
\text { Analysis }\end{array}$ & $\begin{array}{l}\text { Minister of } \\
\text { Finance } \\
\text { Bank of } \\
\text { Mozambique }\end{array}$ \\
\hline & August 2011 & Short-term Expert & Monetary Policy Framework & $\begin{array}{l}\text { Bank of } \\
\text { Mozambique }\end{array}$ \\
\hline & September 2010 & Mission & $\begin{array}{l}\text { Follow-up on Medium-Term } \\
\text { Debt Strategy, Debt Markets, } \\
\text { and Capacity to Conduct Debt } \\
\text { and Project Analysis }\end{array}$ & $\begin{array}{l}\text { Minister of } \\
\text { Finance } \\
\text { Bank of } \\
\text { Mozambique }\end{array}$ \\
\hline & June 2010 & Mission & $\begin{array}{l}\text { Medium-Term Debt Strategy, } \\
\text { Debt Markets, and Capacity } \\
\text { to Conduct Debt and Project } \\
\text { Analysis }\end{array}$ & $\begin{array}{l}\text { Minister of } \\
\text { Finance } \\
\text { Bank of } \\
\text { Mozambique }\end{array}$ \\
\hline & April 2010 & Short-term Expert & $\begin{array}{l}\text { Strengthening Monetary } \\
\text { Policy Formulation and } \\
\text { Implementation }\end{array}$ & $\begin{array}{l}\text { Bank of } \\
\text { Mozambique }\end{array}$ \\
\hline
\end{tabular}




\begin{tabular}{|c|c|c|c|c|}
\hline \multicolumn{5}{|c|}{$\begin{array}{l}\text { IMF Technical Assistance Provided to Mozambique (2005-12) } \\
\text { (As of April 16, 2012) }\end{array}$} \\
\hline \multirow[t]{13}{*}{ Departments } & Timing & Form & Purpose & Counterparts \\
\hline & November 2009 & Short-term Expert & $\begin{array}{l}\text { Strengthening Monetary } \\
\text { Policy Formulation and } \\
\text { Implementation }\end{array}$ & $\begin{array}{l}\text { Bank of } \\
\text { Mozambique }\end{array}$ \\
\hline & May 2009 & Mission & 2009 FSAP Follow up & $\begin{array}{l}\text { Bank of } \\
\text { Mozambique }\end{array}$ \\
\hline & February 2009 & Mission & $\begin{array}{l}\text { TA on Central Banking and } \\
\text { Monetary Policy Formulation }\end{array}$ & $\begin{array}{l}\text { Bank of } \\
\text { Mozambique }\end{array}$ \\
\hline & May 2008 & Mission & $\begin{array}{l}\text { Strengthening Monetary } \\
\text { Policy Formulation and } \\
\text { Implementation }\end{array}$ & $\begin{array}{l}\text { Bank of } \\
\text { Mozambique }\end{array}$ \\
\hline & December 2007 & Mission & $\begin{array}{l}\text { Implementation of IFRS for } \\
\text { Central Bank Accounts. }\end{array}$ & $\begin{array}{l}\text { Bank of } \\
\text { Mozambique }\end{array}$ \\
\hline & August 2007 & Mission & $\begin{array}{l}\text { Post-FSAP TA Assessment: } \\
\text { Banking supervision, } \\
\text { Monetary Policy and } \\
\text { Operations }\end{array}$ & $\begin{array}{l}\text { Bank of } \\
\text { Mozambique }\end{array}$ \\
\hline & April 2007 & $\begin{array}{l}\text { Short-term } \\
\text { consultant }\end{array}$ & Banking Supervision & $\begin{array}{l}\text { Bank of } \\
\text { Mozambique }\end{array}$ \\
\hline & October 2006 & Mission & $\begin{array}{l}\text { Post-FSAP TA Assessment: } \\
\text { Banking supervision, } \\
\text { Monetary Policy and } \\
\text { Operations }\end{array}$ & $\begin{array}{l}\text { Bank of } \\
\text { Mozambique }\end{array}$ \\
\hline & Feb-March 2006 & Mission & AML/CFT legislation & $\begin{array}{l}\text { Bank of } \\
\text { Mozambique }\end{array}$ \\
\hline & Jan-Feb 2006 & $\begin{array}{l}\text { Short-term } \\
\text { consultant }\end{array}$ & Monetary Policy Framework & $\begin{array}{l}\text { Bank of } \\
\text { Mozambique }\end{array}$ \\
\hline & Jan-Feb 2006 & $\begin{array}{l}\text { Short-term } \\
\text { consultant }\end{array}$ & Monetary Operations & $\begin{array}{l}\text { Bank of } \\
\text { Mozambique }\end{array}$ \\
\hline & January 2006 & $\begin{array}{l}\text { Short-term } \\
\text { consultant }\end{array}$ & Exchange Rate Management & $\begin{array}{l}\text { Bank of } \\
\text { Mozambique }\end{array}$ \\
\hline \multirow[t]{5}{*}{ Statistics } & April 2012 & Mission & Government Finance Statistics & $\begin{array}{l}\text { National Institute } \\
\text { of Statistics }\end{array}$ \\
\hline & $\begin{array}{l}\text { March-April } \\
2012\end{array}$ & Mission & Consumer Price Statistics & $\begin{array}{l}\text { National Institute } \\
\text { of Statistics }\end{array}$ \\
\hline & $\begin{array}{l}\text { November- } \\
\text { December } 2011\end{array}$ & Mission & Balance of Payments & $\begin{array}{l}\text { Bank of } \\
\text { Mozambique }\end{array}$ \\
\hline & February 2010 & Mission & Multitopic GDDS & $\begin{array}{l}\text { National Institute } \\
\text { of Statistics }\end{array}$ \\
\hline & March-April & Mission & National Accounts Statistics & National Institute \\
\hline
\end{tabular}




\begin{tabular}{|l|l|l|l|l|}
\hline \multicolumn{5}{|c|}{ IMF Technical Assistance Provided to Mozambique (2005-12) } \\
(As of April 16, 2012)
\end{tabular}




\title{
APPENDIX II. WORLD BANK-IMF COLLABORATION
}

\author{
(As of April 16, 2012)
}

\begin{abstract}
1. The Fund Mozambique team met with the World Bank Mozambique team in Maputo during the fourth PSI review mission in March 2012. The objective was to identify macro-critical structural reforms and to coordinate the two teams' work for the period April 2012 to March 2013.
\end{abstract}

2. The teams agreed that Mozambique's main macroeconomic challenge is to sustain higher and more inclusive economic growth going forward. To meet this challenge, Mozambique needs to enable broader-based economic activity led by the private sector, in particular in areas where it has competitive advantages, such as agriculture, trade-related services in support of booming commodity export sectors, labor-intensive manufacturing, and tourism. Given the low level of infrastructure, this will entail policies and reforms to boost public investment, coupled with enhancements in the business environment. While traditional aid will continue to address needs in infrastructure investment and the social sector, increasing public investment may require resorting to nonconcessional financing and stepping up capacity in the investment selection and debt management areas.

3. Based on this shared assessment, the teams identified seven structural reform areas as macro-critical, in view of their central role in achieving higher and more inclusive growth:

Debt management and investment planning. The authorities' ambitious investment program will need to be backed by a strong reform agenda to enhance capacity to manage fiscal risks and ensure value for money. In the field of investment selection, several measures will be implemented to establish an investment pipeline and define project selection standards and protocols. A new legal framework supporting private-public partnership and concessions is being implemented. Debt management will be strengthened to ensure that borrowing and investment decisions entail value for money. This will be underpinned by a new medium-term debt strategy.

Public financial management. The focus will be on enhancing the capacity to manage risks outside the central government, including in the area of state-owned enterprises (SOEs) and decentralized administrative units. To achieve this objective, the authorities will step up risk-based controls and audits, continue rolling out their new PFM system, and enhance budget execution and monitoring reports. Other critical measures aim at enhancing control of the wage bill, both in budget preparation and execution. These reforms are macro-critical since they will create fiscal space needed to reduce poverty and increase priority spending, while managing risks related to SOE's large-scale investment plans and enhancing the government's oversight.

Tax administration. Despite the strong increase in revenue collections, the tax system is still complex to administer and comply with. It is marked by numerous tax benefits and an overall low contribution from the mega-project sectors. Going forward, the government plans to facilitate tax compliance through electronic tax payment and continue to expand the tax base to cover the large informal sector. The revenue management system will be enhanced, in line with a set of agreed performance indicators. The large tax-payers unit will be made fully operational. 
Social safety nets. Two decades of high GDP growth have not yet resulted in significant poverty reduction. This, in turn, undermines the country's long-term growth potential. To address this, the authorities have started to strengthen their safety nets. Measures include: (i) rationalizing and expanding health insurance coverage; (ii) strengthening higher education and vocational training; (iii) strengthening the financial sustainability and administration of the National Social Security Fund, and creating a new pension system for private sector workers; and (iv) improving the targeting of social protection systems to the most needy. These measures are macro-critical, as better health and education services, stronger pensions systems, and a more effective social safety net can enhance the productivity of the workforce and support social cohesion.

Natural resource wealth. Mozambique is endowed with rich natural resource reserves which are largely not exploited so far. With the recently started coal projects in Tete and a series of other projects in the coming years, the extractive sector is slated for a boom which could make significant contributions to the country's economic growth and government revenues. This poses challenges for economic management, the sector's legal and regulatory frameworks and the government's capacity in managing contracts and revenues. In addition, Mozambique is in the process of attaining full membership of the Extractive Industries Transparency Initiative (EITI).

Investment climate. Mozambique's private sector is held back by the high costs of doing business. These arise mainly from weaknesses in governance, access to finance, power supply, tax and regulatory systems, and contract enforcement. Wide-ranging reforms are needed to address these issues. They are macro-critical because a better investment climate is conducive to supporting private sector activities, thereby broadening the country's productive and export base, creating jobs, and thus helping reduce poverty.

Financial sector stability and development. Strong past growth of credit to the private sector, the expansion of the banking system and its potential exposure to cross-border risks call for strengthening the continuous supervision of the financial sector, as well as the capacity to identity and address potential sources of vulnerabilities. Meanwhile, improving financial intermediation and access to credit remains a top priority. To this end, the authorities have prepared the 2011-20 Financial Sector Development Strategy which includes a wide-ranging and clearly sequenced action plan, covering ways to, among other things, enhance financial inclusion, competition, consumer protection, and financial literacy.

\section{The teams agreed on the following division of labor:}

Debt management and investment planning reforms. The Fund will continue to play a key role in building government capacity to assess debt sustainability and define a coherent debt strategy through TA and continued support from HQ. Both institutions will assist the authorities in strengthening their debt management capacity, with TA support from the Fund and on-site assistance from the Bank. The Fund will continue to play a key role in helping the authorities plan investments, in line with recent TA and program commitments. The Bank will take a lead in enhancing project evaluation and prioritization, and monitoring processes and protocols from its office in Mozambique. The Fund review missions will provide further opportunities to assess progress in this field and discuss possible actions with the Bank. 
Public financial management reform. The Bank and the Fund will cooperate in the field of rolling out the new PFM system to cover local administrations and SOEs engaging in large-scale infrastructure investments. The Fund will secure its support through regular TA missions from its RTAC office. The Bank will provide ongoing consultation from its local office and supervision missions of projects under implementation in this area. The Fund program review missions will provide further opportunities to assess progress in this field.

Tax administration reforms. The Fund will provide support through ongoing advice based on regular TA. The Bank will advance reforms in this area in the context of its business environment policies and portfolio.

Social safety net reform. The Bank will take a lead in this field through continued TA in the design of more comprehensive and performing safety nets, in cooperation with other development partners and international organizations (including UNICEF and ILO). The Fund will play its role in identifying the fiscal space for the safety nets. The results will be discussed among the collaborators on a regular basis, so as to inform policy discussions with the authorities. The Bank is also taking the lead in public pension reforms.

Natural resource wealth. The Fund will provide TA under the newly established Topical Trust Fund for Managing Natural Resource Wealth (TTF-MNRW), relating to the fiscal regime of the extractive sector, revenue forecasting, and natural resource statistics, all being crucial to increase the sector's contribution to the budget and support the country's development agenda. The Bank is supporting Mozambique's implementation of EITI through its Multi-donor Trust Fund (MDTF). The Bank also funds the update of the mining law and regulations and the model mining contract, and the development of a concessions database for gas and mining projects to support the government's forecasting and contract monitoring functions.

Investment climate reform. The Bank will take a lead in working with government to enhance the investment climate, by deepening its agenda to help the authorities cut red tape and simplify the legal and regulatory framework. The results will be shared on an ongoing basis and discussed during the Fund program review missions.

Financial sector stability and development. The Fund will continue to help the Bank of Mozambique to build its capacity to assess risks to financial stability of the banking sector, while the Bank will take the lead in supporting financial sector development and reforms, including through enhanced financial inclusion, competition, consumer protection, and financial literacy. The results of their respective work will be shared and discussed regularly.

5. The teams have the following requests for information from their counterparts:

- $\quad$ The Fund team requests to be kept informed of progress in the above macro-critical structural reform areas. Timing: when milestones are reached; and at least semiannually during Fund program review missions.

- The Bank team requests to be kept informed of the Fund's assessments of macroeconomic policies and prospects, and analytical work on areas under the Fund's lead, as well as on recommendations of Fund TA missions. Timing: in the context of Fund program review and other missions, and at least semi-annually. 
6. The table below lists the teams' separate and joint work programs during April 2012March 2013. 


\begin{tabular}{|c|c|c|c|}
\hline \multicolumn{4}{|c|}{$\begin{array}{l}\text { Mozambique: Bank and Fund planned activities in macro-critical structural reform areas, } \\
\text { April 2012-March } 2013\end{array}$} \\
\hline Title & Products & $\begin{array}{l}\text { Timing of } \\
\text { Missions }\end{array}$ & $\begin{array}{l}\text { Expected } \\
\text { delivery date }\end{array}$ \\
\hline 1. Bank Work Program & $\begin{array}{l}\text { Country Partnership Strategy FY12-15 } \\
\text { FSTAP project support to financial sector reforms } \\
\text { Structural reforms: procurement, decentralization, } \\
\text { business environment, financial sector } \\
\text { Support on targeted, sustainable safety net } \\
\text { measures (including public works system) }\end{array}$ & $\begin{array}{c}- \\
\text { Ongoing } \\
\text { Ongoing }\end{array}$ & $\begin{array}{l}\text { April } 2012 \\
\text { Ongoing } \\
\text { Ongoing }\end{array}$ \\
\hline 2. Fund Work Program & $\begin{array}{l}\text { Fourth PSI Review } \\
\text { Updated macro framework data and PSI review } \\
\text { staff report } \\
\text { TA follow-up mission on PFM (budget execution) } \\
\text { TA follow-up on public accounting } \\
\text { TA on fiscal regime of the extractive sector } \\
\text { TA on price statistics } \\
\text { TA follow-up on GFS fiscal data (GFSM 2001) } \\
\text { TA follow-up mission on tax policy } \\
\text { TA on stress testing } \\
\text { TA on monetary policy formulation } \\
\text { TA on payment system oversight }\end{array}$ & $\begin{array}{l}\text { March } 2012 \\
\text { PSI reviews } \\
\text { Mar-Apr. } 2012 \\
\text { Mar-Apr. } 2012 \\
\text { April } 2012 \\
\text { April } 2012 \\
\text { April } 2012 \\
\text { May } 2012 \\
\text { May } 2012 \\
\text { May } 2012 \\
\text { Sept. } 2012\end{array}$ & $\begin{array}{l}\text { June } 2012 \\
\text { June } 2012 \\
\text { June } 2012 \\
\text { June } 2012 \\
\text { June } 2012 \\
\text { July } 2012 \\
\text { July } 2012 \\
\text { July } 2012 \\
\text { Nov. } 2012\end{array}$ \\
\hline 3. Joint Work Program & $\begin{array}{l}\text { PFM: continue to monitor consolidation and } \\
\text { expansion of e-SISTAFE } \\
\text { Policy advice on public sector salary reforms (incl. } \\
\text { pensions) } \\
\text { Review of tax policy } \\
\text { Policy advice on natural resource wealth } \\
\text { management } \\
\text { Social Protection: Design of programs and } \\
\text { assessment of fiscal space }\end{array}$ & $\begin{array}{l}\text { Ongoing } \\
\text { Ongoing } \\
\text { TBD } \\
\text { Ongoing } \\
\text { Ongoing }\end{array}$ & $\begin{array}{l}\text { Ongoing } \\
\text { Ongoing } \\
\text { TBD } \\
\text { Ongoing }\end{array}$ \\
\hline
\end{tabular}




\section{APPENDIX III. STATISTICAL ISSUES}

\section{Assessment of Data Adequacy for Surveillance}

General: Data provision has some shortcomings, but is broadly adequate for surveillance. Despite the increase in budget resources allocated to the compilation of official statistics, continued high reliance on external funding raises concerns about the sustainability of the programs of the National Institute of Statistics (INE). The authorities are making efforts to improve the quality and timeliness of economic and financial data through participation in the Fund's General Data Dissemination System (GDDS) and in the external sector module of the GDDS Project for Anglophone African Countries (funded by the U.K. Department for International Development (DFID)). Weaknesses exist, particularly in the areas of national accounts, prices, and government finance statistics.

Real sector statistics: The national accounts are prepared by the INE in accordance with the 1993 System of National Accounts (1993 SNA). In August 2007, the INE released quarterly GDP estimates at constant prices covering 2000-07 for the first time, along with a revised annual GDP series for the period 1991-2006, with 2003 as the new base year. The authorities are working to strengthen quarterly national accounts compilation at current prices. The INE's new household income and expenditure survey and economic censuses leading to a new business registry still need to be incorporated to strengthen the national accounts. A revamped consumer price index for Maputo based on weights derived from the 2008-09 household survey was released in February 2011. A national index obtained by integrating the indices for Maputo, Beira, and Nampula was released in April 2006. INE has adopted a new software from INE Portugal which helps to correct a probable downward bias caused by carry-forward techniques for discontinued products. Several issues remain outstanding, however, including how to improve the housing market rent sample and expand coverage to include the Quelimane and Greater Maputo areas. There are insufficient sectoral labor market and employment data, and where available, they have limited coverage. A one-year labor market survey of the entire country, undertaken by INE in collaboration with the Labor Ministry, was launched in October 2004.

Monetary and financial statistics: A 2004 work plan was developed to implement the Monetary and Financial Statistics Manual (MFSM) and develop an integrated monetary database (IMD) to meet the needs of the Bank of Mozambique (BM), AFR, and STA. In November 2007 the IMD was completed and allows the derivation of accurate and timely monthly monetary statistics, while reducing BM's reporting burden.

Balance of payments: With assistance from STA, provided in the context of the GDDS regional project, the BM has made significant progress toward compiling and disseminating balance of payments (BOP) and international investment position (IIP) statistics that are fully aligned with the Balance of Payments Manual, fifth edition (BPM5). The BM has an adequate institutional framework for the compilation of BOP and IIP statistics. However, further strengthening of external sector statistics needs to focus on: strengthening information technology (especially to apply data warehouse and on-line analytical processing tools); excluding the effects of price and 
exchange rate fluctuations from reserve asset flows; applying a methodology (provided through STA technical assistance) to estimate reinvested profits and stocks of financial assets and liabilities held abroad by the nonfinancial public sector; improving business surveys; publishing methodological notes, in general, and more data covering the Reserves Template, foreign direct investment, goods trade and current transfers; and beginning the gradual process to implement the new Balance of Payments and International Investment Position Manual, sixth edition. Joint work with other institutions to improve the quality of external trade data, especially with regard to price and volume indices, also merits attention.

\section{Data Standards and Quality}

Mozambique commenced its participation in the General Data Dissemination System (GDDS) in November 2003; but has since then not updated much of its metadata.
In May 2005, an update of the June 2002 data module of the Report on the Observance of Standards and Codes (Data ROSC) was prepared. Improvements in the institutional environment and increased allocation of resources for the compilation of national accounts and balance of payments statistics aimed to address weaknesses in the prerequisites for the quality of the statistics. Methodological soundness, accuracy, and reliability of macroeconomic statistics began to show improvements as a result of these efforts.

\section{Reporting to STA}

Mozambique does not report fiscal data for publication in the IFS or the Government Finance Statistic Yearbook (GFSY). Mozambique now reports monthly monetary data to STA using the standardized reporting forms (SRFs). As a result, these enhanced data are being published in the International Financial Statistics (IFS) Supplement. In 2007, the BM started to compile and report comprehensive IIP data in the Balance of Payments Statistics Yearbook. 


\section{Mozambique: Table of Common Indicators Required for Surveillance}

(As of April 16, 2012)

\begin{tabular}{|c|c|c|c|c|c|c|c|}
\hline & Date of latest & & Frequency of & Frequency of & & & ems: \\
\hline & observation & received & Data $^{7}$ & Reporting $^{7}$ & $\begin{array}{c}\text { of } \\
\text { publication }^{7}\end{array}$ & $\begin{array}{c}\text { Data Quality - } \\
\text { Methodological } \\
\text { soundness } \\
\end{array}$ & $\begin{array}{l}\text { Data Quality Accuracy } \\
\text { and reliability }\end{array}$ \\
\hline Exchange Rates & March 2012 & March 2012 & $\mathrm{D}$ & W & $\mathrm{D}$ & & \\
\hline $\begin{array}{l}\text { International Reserve Assets and Reserve } \\
\text { Liabilities of the Monetary Authorities }^{1}\end{array}$ & March 2012 & March 2012 & $D$ & W & $\mathrm{D}$ & & \\
\hline Reserve/Base Money & March 2012 & April 2012 & M & M & M & LO, O, LO, O & LO, LO, O, O, LO \\
\hline Broad Money & Feb. 2012 & April 2012 & M & M & M & & \\
\hline Central Bank Balance Sheet & Feb. 2012 & April 2012 & M & M & M & & \\
\hline $\begin{array}{l}\text { Consolidated Balance Sheet of the Banking } \\
\text { System }\end{array}$ & Feb. 2012 & April 2012 & M & M & M & & \\
\hline Interest Rates ${ }^{2}$ & March 2012 & April 2012 & M & M & M & & \\
\hline Consumer Price Index & March 2012 & April 2012 & M & M & M & O, LO, LNO, O & LNO, LO, LO, O, O \\
\hline $\begin{array}{l}\text { Revenue, Expenditure, Balance and } \\
\text { Composition of Financing }^{3}-\text { General } \\
\text { Government }^{4}\end{array}$ & Dec. 2011 & Feb. 2012 & $M, Q$ & Q & $\mathrm{Q}$ & LO, LNO, LO, O & LO, O, LO, O, LNO \\
\hline $\begin{array}{l}\text { Stocks of Central Government and Central } \\
\text { Government-Guaranteed Debt }\end{array}$ & 2011 Q4 & March 2012 & Q & Q & $\mathrm{Q}$ & & \\
\hline External Current Account Balance & 2011 Q4 & Feb. 2012 & Q & I & I & LO, LNO, LO, O & LO, LNO, LO, LO, LNO \\
\hline Exports and Imports of Goods and Services & 2011 Q4 & Feb. 2012 & Q & I & I & & \\
\hline GDP/GNP & 2011 Q4 & March 2012 & Q & Q & I & O, LO, O, LO & LNO, LO, LO, O, LO \\
\hline Gross External Debt & Dec. 2010 & Oct. 2011 & A & I & I & & \\
\hline International Investment Position ${ }^{6}$ & 2007 & May 2009 & A & A & A & & \\
\hline
\end{tabular}

${ }^{1}$ Any reserve assets that are pledged of otherwise encumbered should be specified separately. Also, data should comprise short-term liabilities linked to a foreign currency but settled by other means as well as the notional values of financial derivatives to pay and to receive foreign currency, including those linked to a foreign currency but settled by other means.

${ }^{2}$ Both market-based and officially determined, including discount rates, money market rates, rates on treasury bills, notes, and bonds.

${ }^{3}$ Foreign, domestic bank, and domestic nonbank financing.

${ }^{4}$ The general government consists of the central government (budgetary funds, extra budgetary funds, and social security funds) and state and local governments.

${ }^{5}$ Including currency and maturity composition.

${ }^{6}$ Includes external gross financial asset and liability positions vis-à-vis nonresidents.

${ }^{7}$ Daily (D); Weekly (W); Monthly (M); Quarterly (Q); Annually (A); Irregular (I); Not Available (NA).

${ }^{8}$ Reflects the assessment provided in the data ROSC published in August, 2008, and based on the findings of the mission that took place during November 29-December 7, 2007 for the dataset corresponding to the variables in each row. The assessment indicates whether international standards concerning concepts and definitions, scope, classification/sectorization, and basis for recording are fully observed (O), largely observed (LO), largely not observed (LNO), or not observed (NO).

${ }^{9}$ Same as footnote 8, except referring to international standards concerning source data, assessment and validation of source data, statistical techniques, assessment and validation of

intermediate data and statistical outputs, and revision studies. 
Press Release No. 12/206

FOR IMMEDIATE RELEASE

June 6, 2012
International Monetary Fund

Washington, DC

\section{IMF Executive Board Completes Fourth Review Under Policy Support Instrument for Mozambique}

The Executive Board of the International Monetary Fund (IMF) today completed the fourth review of Mozambique's economic program under the Policy Support Instrument (PSI). In completing the review, the Board approved a waiver for the minor nonobservance of the end-December 2011 assessment criterion on net credit to the government.

The Executive Board approved Mozambique's second three-year PSI on June 14, 2010 (see Press Release No. 10/242). The IMF's framework for PSIs is designed for low-income countries that may not need, or want, IMF financial assistance, but still seek IMF advice, monitoring, and endorsement of their policies (see Public Information Notice No. 05/145).

At the conclusion of the Executive Board discussion on Mozambique, Mr. Min Zhu, Deputy Managing Director and Acting Chair, issued the following statement:

"Mozambique's sound policies and strong economic performance are commendable. Economic growth remained buoyant in 2011 and inflation was successfully contained. Mozambique's prospects for economic growth over the medium term are good, but short-term risks from the global environment should be properly managed.

"The authorities appropriately envisage a countercyclical policy mix for 2012 to sustain economic growth. They aim to keep spending at budgeted levels and pursue an accommodative monetary policy. It will be important to safeguard the recent disinflation gains. 
"The authorities' Poverty Reduction Strategy (PARP) for 2011-14 aims to reinvigorate poverty reduction. The authorities have initiated measures in key areas, including reforms in agriculture and the business environment, continued social and human development, and the expansion of a well-targeted and affordable social protection floor. It will be important to implement these plans vigorously to allow more Mozambicans to benefit from the expected strong economic growth.

"The authorities' ambitious structural reform agenda requires addressing pressing infrastructure needs. It will be important to maintain the reform momentum to strengthen debt management, investment planning, and public financial management to aid in informed borrowing and project selection decisions, given the authorities' plans to have greater recourse to nonconcessional resources.

"Proper management of the mining and emerging hydrocarbon sectors, together with a strong tax administration effort, will help enhance fiscal resources for infrastructure and other medium- to longterm development needs. The authorities' plans to move rapidly in modernizing the fiscal regimes for mining and petroleum and to obtain full EITI (Extractive Industries Transparency Initiative) membership go in the right direction," Mr. Zhu added. 
Statement by Mr. Momodou Bamba Saho, Alternate Executive Director for Mozambique June 6, 2012

\section{Introduction}

My authorities are thankful to Management and Executive Directors for the continued support that is enabling Mozambique weather the global economic uncertainties relatively well. The implementation of their Poverty Reduction Strategy (PARP) for 2011 - 2014, supported by the Policy Support Instrument (PSI) is helping sustain the growth momentum through the implementation of well-balanced macroeconomic policies and good policy advice. They thank staff for the candid discussions on recent economic developments in Mozambique and challenges and opportunities.

\section{Program performance}

Mozambique's overall performance under the PSI has been strong, reflecting prudent macroeconomic policies and the authorities' commitment to maintain sustained and inclusive economic growth. All end-December 2011 quantitative assessment criteria (ACs) were comfortably met with the exception of net credit to the government (NCG) which was marginally exceeded because of an unanticipated call on a government guaranty for a public enterprise. However, the Mozambican' authorities are committed to undertake corrective actions in order to observe the agreed target in the next review. The authorities' strong action in slowing reserve money growth in 2011 contributed to lower than programmed inflation in end-December 2011. The net international reserves target was also met as well as the indicative target on priority spending. They also met the structural benchmark on approval of the Integrated Investment Program that was designed to prioritize and improve public investment project selection.

\section{Economic prospects and policies}

Despite an adverse external environment and after being affected by natural disasters earlier in 2012 which damaged infrastructures, Mozambique largely met the objectives underlying its economic program and remain confident of sustained economic growth while continuing to further disinflate the economy. Mozambique remains relatively resilient to shocks, and inflation is well anchored. Growth was estimated at 7.1 percent in 2011, driven by favorable developments in international prices and continuous boom in the mineral sector. It is projected to remain elevated in the medium term although the global slowdown poses some risks to the outlook. As a result of 
strong action from the authorities in tightening monetary and fiscal policies, headline inflation declined from double digits in 2009 and in 2010 to around 5 percent at end- 2011 and 2.5 percent in February 2012. Exports and FDI have remained robust in spite of the less favorable external environment, resulting in a better than expected level of international reserves.

\section{Fiscal policy}

My authorities are committed to implement their fiscal reform program despite a weakening global environment and the prospects of declining donor support. Fiscal policy in 2012 will be countercyclical. Revenues may be less than the estimates contained in the 2012 budget law if downside risks from the global economic scenario materialize. To cushion the economy from these effects and to meet the objectives of the PARP, expenditures will remain as programmed. This may result in an increase in the primary deficit by 0.2 percent to 2.3 percent of GDP. This increase would be fully financed by additional World Bank financing.

In order to make economic growth more inclusive and protect the poor and the vulnerable, a significant proportion of spending will be directed at increasing agricultural production, promoting employment creation and investment in human social development. Some 53 percent of projected total expenditures, minus net lending, will be targeted towards the PARP priority areas. Social safety nets would be strengthened with the expansion of the cash transfer mechanism to the most vulnerable groups and implementation of the public works programs. In addition, the authorities are committed to wage restraint and fully eliminating the fuel subsidy.

\section{Monetary and financial sector developments}

To mitigate the impact of the global downturn, monetary policy in 2012 will continue to be accommodative. However, the Bank of Mozambique (BM) remains committed to meeting its inflation objective. The monetary stance will help buttress private sector credit, allowing the sector to continue to contribute to Mozambique's economic growth. The authorities are also committed to reform the monetary policy framework. As highlighted by staff, they continue to make progress in preparing for an inflation targeting framework in the medium term. Meanwhile, the Ministry of Finance and BM continue to strengthen their close collaboration to improve the current framework for monetary operations. 
Towards this end, the two institutions continue to improve information sharing in line with the spirit of their MoU signed in March 2011, helping BM improve liquidity forecasting. In addition, with the help of Fund TA, the BM continues to develop the domestic repo market to improve money market management and liquidity of government securities.

The authorities are committed to maintaining the flexible exchange rate regime and monitoring the foreign exchange market to ensure that regulations and practices are consistent with Article VIII principles.

In response to the uncertain external environment, $\mathrm{BM}$ is improving its financial sector surveillance mechanisms with the help of Fund TA. These include further assessments of risks arising from turmoil in the euro area, steps to enhance risk-based supervision and completion of a financial sector contingency plan. The authorities will also consider options to make the Deposit Insurance Fund operational. The AML/CFT law, an integral part of the AML/CFT framework, was submitted to parliament on April 19, 2012. The authorities expect the framework to be operational soon.

\section{Implementation of the PARP 2011-2014}

My authorities are aware that more still to be done in order to reduce poverty as underlined in the Joint Staff Advisory Note. Building on existing efforts, economic growth needs to continue enabling the structural transformation and diversification of the economy, sustaining the implementation of policies to unlock Mozambique's vast economic potential to support the growth momentum and create employment through improvements in human and institutional capacities. The authorities aim to double agricultural production by 2020 through implementation of their Agricultural Reform Strategy (PEDSA). In this regard, they have taken steps to improve agricultural production and productivity, strengthen the legal mechanisms to facilitate access to land, improve the quality of seeds and fertilizers as well as enhance techniques of production and market access. To boost private sector activity and employment creation, the authorities are implementing measures to improve the business climate and reduce the cost of doing business. These include a decree simplifying procedures for 84 types of licenses and approving an action plan to reduce by half the number of procedures required to start a business, enact a bankruptcy code, complete an inventory of business licenses and approve a credit registry model. They are committed to continued increased public expenditure for priority projects aimed at improving social safety nets, increasing employment and bridging the infrastructure gap. 


\section{Structural reforms}

\section{The Mozambican authorities continue to be strongly committed to implementing the} necessary structural reforms aimed at improving control in budget execution. Implementation of the public financial management system has progressed well, but timely donor support and TA will be important for its completion. In this regard, they plan to complete implementation of their eSISTAFE budgeting system by 2015 covering some 98 percent of spending. For the current year, it is projected that such coverage would be increased to 47 percent. This is complemented by efforts to achieve full compliance with real time recording of the expenditure chain by 2013 and migration of the public accounts to an international public financial accounting standards compatible format. Realizing the importance of enhancing the quality of PFM to addressing the country's challenges, the authorities will persevere in expanding fiscal decentralization and modernizing debt management and investment planning as well as completing the first annual domestic borrowing plan for 2013. The plan will give market participants a clearer signal of the authorities' borrowing intentions enabling better planning by both Government and the market. Reforms in tax administration are also underway with the objectives of improving efficiency and sustaining strong revenue mobilization. Among other initiatives, implementation of a single taxpayer database and registration number, tax payments through banks and strengthening the large taxpayers unit should allow for further improvements in tax collection.

\section{Given the very strong prospects for economic transformation through efficient and prudent management of natural resources, improving capacity in this area remains vital for}

Mozambique. My authorities seek to implement reforms to their mining and hydrocarbon fiscal regime that will allow the country to get the maximum possible benefits from new projects without jeopardizing it's attractiveness as an investment destination. In this regard, they appreciate prospective Fund TA under the Topical Trust Fund for Managing Natural Resources Wealth. For existing natural resources sector projects, where necessary, the authorities will carefully search for prospects for mutually beneficial renegotiations of terms without prejudice to investors. This will enable Mozambique to get an appropriate share of revenue accruing from natural resources wealth and increase the contribution of the sector to economic development. The authorities are committed to prudent and transparent management of these resources for the benefit of their population. They have consequently made significant progress towards full EITI membership. 


\section{Conclusion}

My authorities would like to reiterate their commitment to sound macroeconomic policies. They value the Fund advice and TA that is helping Mozambique maintain macroeconomic stability and move towards sustained economic growth. In view of the solid performance under the program, the Mozambican authorities seek the Executive Board's approval of their request for the completion of the fourth review under the PSI and the related waiver for the nonobservance of the end-2011 AC on NCG, and for modifications of end-June 2012 ACs. 